\title{
CLASSIFICATION OF VENTRICULAR ARRHYTHMIAS BASED ON A DATA DRIVEN APPROACH
}

\author{
by \\ Matthew Hotradat, \\ B.Eng., Ryerson University, June 2016
}

A thesis

presented to Ryerson University

in partial fulfillment of the requirements for the degree of

Master of Applied Science in the program of

Biomedical Engineering

Toronto, Ontario, Canada, 2019

(C) Matthew Hotradat, 2019 


\section{AUTHOR'S DECLARATION FOR ELECTRONIC SUBMISSION OF A THESIS}

I hereby declare that I am the sole author of this thesis. This is a true copy of the thesis, including any required final revisions, as accepted by my examiners.

I authorize Ryerson University to lend this thesis to other institutions or individuals for the purpose of scholarly research.

I further authorize Ryerson University to reproduce this thesis by photocopying or by other means, in total or in part, at the request of other institutions or individuals for the purpose of scholarly research.

I understand that my thesis may be made electronically available to the public. 


\section{Abstract \\ Classification of Ventricular Arrhythmias Based on a Data Driven Approach, Master of Applied Science, 2019, \\ Matthew Hotradat, Biomedical Engineering, Ryerson University.}

Ventricular arrhythmias (VA) are dangerous pathophysiological conditions affecting the heart which evolve over time resulting in different manifestations such as ventricular tachycardia (VT), organized VF (OVF), and disorganized VF (DVF). Success of resuscitation for patients is greatly impacted by the type of VA and swift administration of appropriate therapy options. This thesis attempts to arrive at computationally efficient, data driven approaches for classifying and tracking VAs over time for two purposes: (1) 'in-hospital' scenarios for planning long-term therapy options, and (2) 'out-of-hospital' scenarios for tracking progression/segregation of VAs in near real-time.

Using a database of 61 60-s ECG VA segments, maximum classification accuracies of 96.7\% (AUC=0.993) and 87\% (AUC=0.968) were achieved for VT vs. VF and OVF vs. DVF classification for 'in-hospital'/offline analysis. Two near real-time approaches were also developed for 'out-of-hospital' VA incidents with results demonstrating the high potential to track VA progression and segregation over time. 


\section{Acknowledgments}

I gratefully acknowledge my supervisor Dr. K. Umapathy for his consistent guidance and support. I also thankfully acknowledge Dr. K. Nair, and Mr. S. Masse from Toronto General Hospital, University Health Network, for their invaluable clinical expertise during the undertaking of this thesis.

I also thank all of my research lab colleagues and friends at Ryerson University for their support and encouragement.

Lastly, I lovingly thank God and my family for unwavering support and inspiration during my studies. I could not have done it without them. 


\section{Table of Contents}

Declaration

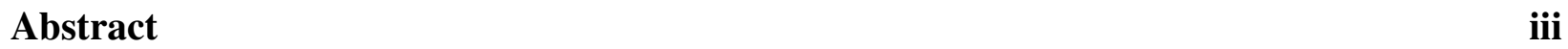

List of Tables

List of Figures

1 Introduction $\quad 1$

1.1 Electrophysiology of the Heart . . . . . . . . . . . . . . . . 2

1.2 Cardiac Ventricular Arrhythmias .................. 3

1.3 Existing Works . . . . . . . . . . . . . . . . . 4

1.4 Motivation . . . . . . . . . . . . . . . . . 7

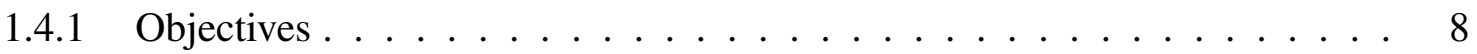

1.5 Thesis Outline . . . . . . . . . . . . . . . . . . 8

2 Background $\quad 10$

2.1 Time-Frequency Analysis . . . . . . . . . . . . . . . . . . 10

2.1.1 Short-Time Fourier Transform . . . . . . . . . . . . . . . . . 11

2.1 .2 Wavelet Analysis . . . . . . . . . . . . . . . . . . 12

2.1.3 Time-Frequency Energy Distributions . . . . . . . . . . . . . . . 15

2.2 Data Driven Decomposition Techniques . . . . . . . . . . . . . . . 18

2.2.1 Empirical Mode Decomposition . . . . . . . . . . . . . . . . . . . 19

2.2.2 Additional EMD-based Methods . . . . . . . . . . . . . . . . 23

2.2.3 Hilbert Energy Spectrum and TFR . . . . . . . . . . . . . . . . 25

2.2.4 Instantaneous Features . . . . . . . . . . . . . . . . . 27

2.3 Pattern Classification and Statistical Analysis . . . . . . . . . . . . . . . . . . . 29

2.3.1 Fisher's Linear Discriminant Analysis . . . . . . . . . . . . . . . . . . . 30

2.3 .2 Cross-Validation . . . . . . . . . . . . . . . . 32

2.3.3 Classification Accuracy . . . . . . . . . . . . . . . . . . 32

2.3.4 Receiver Operating Characteristic . . . . . . . . . . . . . . . 33

2.4 Chapter Summary . . . . . . . . . . . . . . . . . . . . . 34 
$3 \quad$ EMD-IMF Energy Features for VA Classification 35

3.1 Methodology . . . . . . . . . . . . . . . . . . . 36

3.1.1 ECG Database \& Pre-processing . . . . . . . . . . . . . . . . 36

3.1 .2 EMD \& Extracting IMFs . . . . . . . . . . . . . . . . . . . . 37

3.1 .3 Feature Extraction . . . . . . . . . . . . . . . . . . . . 40

3.1.4 Pattern Classification . . . . . . . . . . . . . . . . . . . . . 43

3.2 Results . . . . . . . . . . . . . . . . . . . . . . . 44

3.2.1 Long-Term (In-Hospital or Offline) Analysis . . . . . . . . . . . . . 44

3.2.2 Short-Term (Out-of-Hospital) Analysis . . . . . . . . . . . . . . 52

3.3 Discussion . . . . . . . . . . . . . . . . . 56

3.4 Chapter Summary . . . . . . . . . . . . . . . . . . . . . . . . . . . 59

4 EMD Instantaneous Features for VA Classification and Trends Analysis 61

4.1 Methodology . . . . . . . . . . . . . . . . . 62

4.1.1 ECG Database \& Pre-Processing . . . . . . . . . . . . . . . . . 62

4.1.2 Constructing the Hilbert Energy Spectrum . . . . . . . . . . . . . . 63

4.1 .3 Feature Extraction . . . . . . . . . . . . . . . . . . 64

4.1 .4 Pattern Classification . . . . . . . . . . . . . . . . . . . . 67

4.1.5 Scoring Index Calculation . . . . . . . . . . . . . . . . 67

4.2 Results . . . . . . . . . . . . . . . . . . . . . . 70

4.2.1 Long-Term (In-Hospital) Analysis . . . . . . . . . . . . . . . . 70

4.2 .2 VA Trends Analysis . . . . . . . . . . . . . . . . . 76

4.2.3 Long-Term Analysis Combining Non-Instantaneous and Instantaneous Features . . . . . . . . . . . . . . . . . . 77

4.3 Discussion . . . . . . . . . . . . . . . . . . . . 80

4.4 Chapter Summary . . . . . . . . . . . . . . . . . . . . . . . . 84

5 Conclusions $\quad \mathbf{8 6}$

$5.1 \quad$ Summary of Results . . . . . . . . . . . . . . . . . . . . . . . . . . . 87

5.2 Potential Applications . . . . . . . . . . . . . . . . . . . . . . . . . . 89

5.3 Future Works . . . . . . . . . . . . . . . . . . . . . 89

$\begin{array}{ll}\text { A HHT of Synthetic Signals } & 90\end{array}$

$\begin{array}{lr}\text { References } & 92\end{array}$

$\begin{array}{ll}\text { Glossary } & 99\end{array}$ 


\section{List of Tables}

3.1 VT vs. VF classification accuracy for leave-one-out cross-validated data. . . . . . . 46

3.2 OVF vs. DVF classification accuracy for leave-one-out cross-validated data. . . . . 49

3.3 VT vs. VF classification accuracy for the comparative features. . . . . . . . . . . 51

3.4 OVF vs. DVF classification accuracy for the comparative features. . . . . . . . . . 51

3.5 Classification table for 20 -s, 40 -s, and 60 -s duration results using leave-one-out cross-validated data. . . . . . . . . . . . . . . . . . . 54

3.6 Comparative results classification table for 20 -s, $40-\mathrm{s}$, and 60 -s duration results using leave-one-out cross-validated data . . . . . . . . . . . . . . . . 55

4.1 VT vs. VF classification accuracy for instantaneous features using leave-one-out cross-validated data. . . . . . . . . . . . . . . . . . . . . . 72

4.2 OVF vs. DVF classification accuracy for instantaneous features using leave-oneout cross-validated data. . . . . . . . . . . . . . . . . . . . . . 75

4.3 VT vs. VF classification accuracy for combined features with leave-one-out crossvalidated data. . . . . . . . . . . . . . . . . . . . . . . 78

4.4 OVF vs. DVF classification accuracy for combined features with leave-one-out cross-validated data. . . . . . . . . . . . . . . . . . . . . 79 


\section{List of Figures}

1.1 A sample 3-s ECG signal during normal sinus rhythm. . . . . . . . . . . 3

1.2 Sample ECG waveforms for VT (row 1), OVF (row 2), and DVF (row 3) over 10-s duration. . . . . . . . . . . . . . . . . . 5

1.3 Block diagram outlining proposed approached of this study. . . . . . . . . . . . 9

2.1 A signal composed of a Gaussian atom combined with a complex sinusoid comparing its TFR for WVD, PWVD, and SPWVD . . . . . . . . . . . . . 16

2.2 Empirical mode decomposition of a 60-s OVF sample, showing the first 5 decomposed IMFs. . . . . . . . . . . . . . . . . . . . . 20

2.3 The plot of a VF sample with its Hilbert Energy Spectrum below it. . . . . . . . . 27

2.4 The plot of a VF sample with along with its 3 instantaneous features: IF, $I B^{2}$, and IE. . . . . . . . . . . . . . . . . . . . . . . . . . 29

3.1 Block diagram highlighting the proposed work discussed in this chapter. . . . . . 36

3.2 Sample ECG segments for VT, OVF and DVF from the proposed database. . . . . 37

3.3 The sliding (1-s) windowed ERV values for a sample VT, OVF, and DVF signal. . . 42

3.4 The boxplots for the 3 features used for the VT vs. VF classifier. . . . . . . . . . . 45

3.5 The ROC curve for the VT vs. VF classifier. . . . . . . . . . . . . . . . . . . 48

3.6 The boxplot for the ERS median (1s) feature between OVF and DVF groups. . . . 49

3.7 The ROC curve for the OVF vs. DVF classifier. . . . . . . . . . . . . . 50

4.1 Block diagram highlighting the proposed work discussed in Chapter 4 . . . . . . 62

4.2 Three sample VA segments (VT, OVF, and DVF) with their extracted IF and $I B^{2}$ pictured underneath them. The IF and $I B^{2}$ were low-pass filtered for better visual illustration. . . . . . . . . . . . . . . . . . 65

4.3 The boxplots for the 4 features used to train the VT vs. VF classifier. . . . . . . . 71

4.4 The ROC curve for the VT vs. VF classifier. . . . . . . . . . . . . . . . . . . 73

4.5 The boxplots for the 4 features used to train the OVF vs. DVF classifier. . . . . . 74

4.6 The ROC curve for the OVF vs. DVF classifier. . . . . . . . . . . . . . . 75

4.7 The plot of a VF sample along with its VA trend signal shown below. . . . . . . . . 77

4.8 The ROC curve for the combined VT vs. VF classifier. . . . . . . . . . . . . . . 79

4.9 The ROC curve for the combined OVF vs. DVF classifier. . . . . . . . . . . 80 


\section{Chapter 1}

\section{Introduction}

$7 \mathrm{HE}$ heart is one of the most important organs in the human body, and without its proper

functioning, our quality of life and health would be greatly diminished. It performs many vital functions, the most important of which is the circulation of blood throughout the body. The circulation of blood allows for the gaseous exchange of oxygen and carbon dioxide, as well as transportation of many other essential nutrients to vital organs and tissues. The heart facilitates this circulation by a constant and rhythmic pumping. The heart performs organized and rhythmic contractions of its various chambers, which are stimulated by electrical impulses causing the cardiac muscles to contract and relax. This creates the required pressure and rhythm to allow for blood to circulate to all organs and tissues and return to the heart.

If this natural rhythm and pumping goes awry, it can have serious consequences. The most devastating of all conditions that could result from malfunctioning of the heart is sudden cardiac death (SCD). In fact, SCDs are the cause of 350,000 deaths annually in North America alone, and most of these cases are related to ventricular fibrillation (VF), a major cardiac arrhythmia [1]. Cardiac arrhythmias, particularly ventricular arrhythmias (VA) like VF, are lethal conditions of the heart that are not well understood despite decades of research. As a result, the mortality rate of those suffering from VF remains high, and so improvements to the current knowledge base and available treatment options are major priorities. 
From a signal processing perspective, there have been many existing works which have used electrocardiogram (ECG) signal analysis to assist clinicians and emergency medical staff (EMS) in combating SCDs. ECG recordings during ventricular arrhythmias contain valuable information about the conditions of the heart. Since time is a crucial factor in diagnosing and treating VAs, near real-time analysis of ECG signals are more suitable than other cardiac data which often require time-consuming acquisition methods like imaging.

\subsection{Electrophysiology of the Heart}

Before beginning with the proposed methodology, some background information on the physiology of the heart and cardiac arrhythmias will provide useful context. The resource used for the heart's physiology can be found in [2]. The heart consists of four total chambers: two atria above and two ventricles below. Blood that is returning to the heart from the body enters it via the right atrium. The right atrium then pumps the blood into the right ventricle, which then proceeds to pump the blood to the lungs where it receives oxygen and dispels carbon dioxide. The re-oxygenated blood returns to the left atrium, which then fills the powerful left ventricle. The left ventricle finally pumps the oxygen-rich blood out to the rest of the body (and the cardiac muscles themselves). The two atria contract first, followed by the contraction of the ventricles. The organized contractions of the heart are controlled by electrical impulses. The electrical impulses first travel through the atria, then it propagates through to the atrio-ventricular (AV) node, before it conducts down the Purkinje fibers to the rest of the cardiac muscles, depolarizing the tissue and causing the heart to contract. The order and timing by which the various chambers of the heart contract is precisely controlled autonomically.

The electrical activity of the heart can be measured by an ECG. An ECG is an electrical signal recorded by placing electrodes on the surface of the body around the heart, and the potential difference between specific points is measured. The normal contraction of the heart typically has a very structured and repeatable waveform called the 'PQRST' waveform. A sample ECG signal depicting the normal sinus rhythm (NSR) of the heart is shown in Figure 1.1. The ECG waveform 
gives insight into the behavior of the heart at various stages during its contractions. Likewise, when the normal ECG signal structure is deviated, it indicates an anomaly in the functioning of the heart. Causes of this malfunctioning could be disruptions to the electrical conduction pathway of the heart by pathological conditions or physical damage, and may have serious implications for the health of patients.

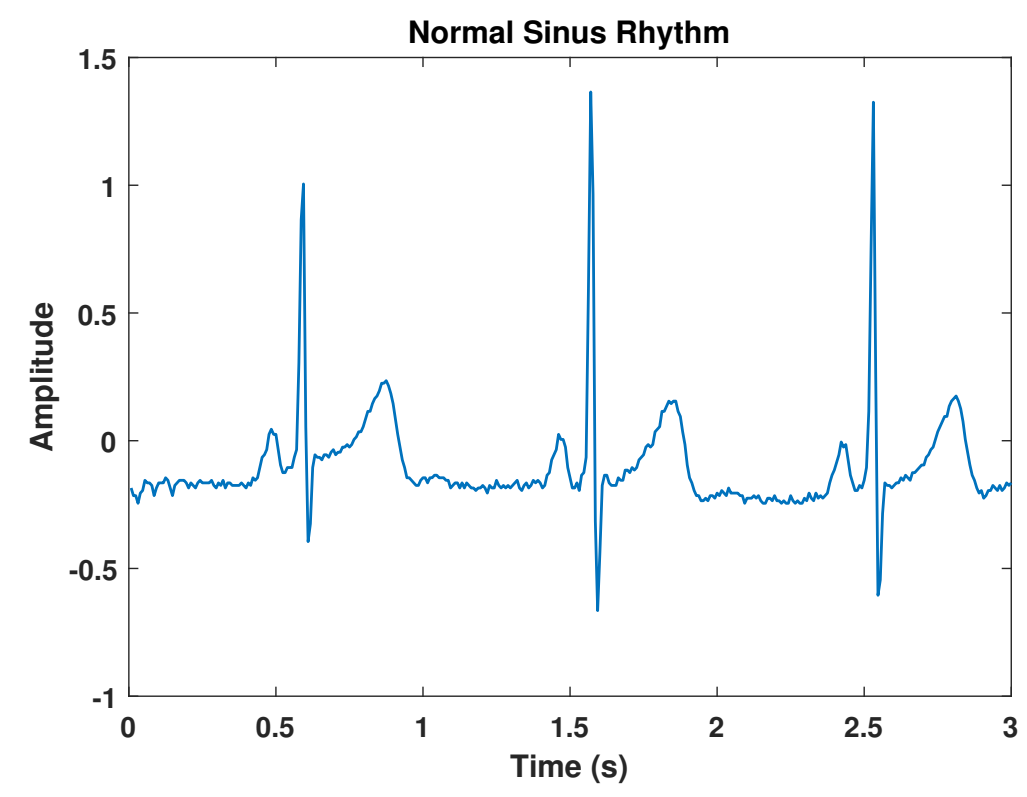

Figure 1.1: A sample 3-s ECG signal during normal sinus rhythm.

\subsection{Cardiac Ventricular Arrhythmias}

Disruptions to the normal electrical conduction system of the heart lead to cardiac arrhythmias [2]. An arrhythmia is characterized by two factors: the location of the disruption within the heart, and the speed or type of rhythm that results from it. For instance, ventricular tachycardia (VT) is an arrhythmia that originates and affects the cardiac ventricles and results in a faster pacing compared to NSR. VAs are the more dangerous type of cardiac arrhythmia, because the ventricles are responsible for pumping blood out to the body, whereas the atria act more as primers used to fill the ventricles with more blood to pump. 
The two major forms of ventricular arrhythmias are: VT and VF. VT results in the cardiac ventricles contracting much faster than normal [2]. When this occurs, the atria are not able to fill the ventricles with sufficient amounts of blood before they contract, resulting in a dramatic decrease in cardiac function. VT will often devolve into VF, which is an even more serious condition. During VF, the cardiac ventricles fail to contract at all, and instead quiver uselessly. This means that virtually no blood is being circulated to the body's vital organs. At the onset of VF, patients only have a few minutes to receive medical attention before it becomes fatal (SCD). Given the severity of these two arrhythmias, they will be the focal points of this thesis.

Despite decades of research, the underlying mechanisms that underpin VF are still not fully understood. To gain a better understanding of VF and for optimizing treatment options, previous works have tried to sub-categorize VF based on specific characteristics. Some have tried to use the temporal evolution of VF to separate it into various stages [3-5]. Others have used the spatiotemporal organization of VF to categorize it into different sub-types. This is important because the organization of VF has shown to be related to the underlying rotor/wavefront mechanisms believed to sustain it $[6,7]$.

VF can also be further subdivided into the following 2 categories: organized VF (OVF) and disorganized VF (DVF) [8]. This differences between OVF and DVF comes down to subtle variations in temporal complexity, and discriminating them is a challenging problem. It was demonstrated that temporal complexity is related to the underlying mechanisms believed to sustain VF [9]. OVF and DVF tend to overlap in nature, and will often evolve into each other over time. Since different treatment options are appropriate for treating each of them, characterizing and detecting these 2 VF-subtypes accurately and swiftly is also critical to improving the survival rate of patients.

\subsection{Existing Works}

One of the challenges of studying VAs, and particularly VF, is its temporal and spectral fluctuations over time. This non-stationary nature makes it challenging to quantify the behaviour of VF over 

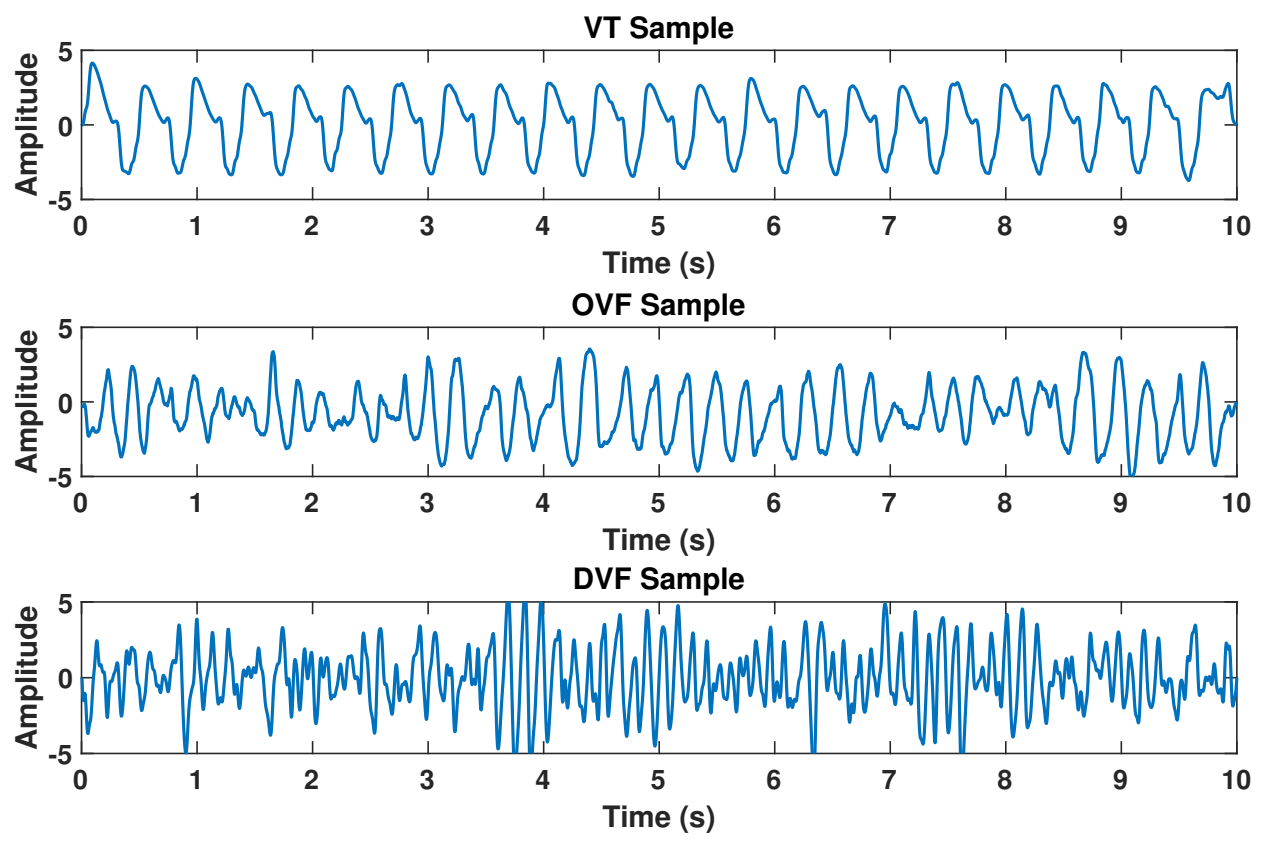

Figure 1.2: Sample ECG waveforms for VT (row 1), OVF (row 2), and DVF (row 3) over 10-s duration.

time using time-averaged analysis methods. Thus, using a signal analysis technique capable of capturing time-varying spectral content is ideal. However, many different signal analysis approaches have been used over the years to study VAs. Some of the earliest studies used simple time-domain methods to analyze the ECG signals. For example, features like threshold crossing interval, autocorrelation, and complexity measure were extracted to quantify arrhythmias [10]. The issue with time-domain analysis is that many of the underlying structures of VAs are not readily perceivable in the time-domain. Researchers turned to frequency-domain analysis, where differences in spectral content could be observed. Parameters like VF filter leakage [10], median frequency, and dominant frequency (DF) were effective at characterizing VF and resuscitation success in patients suffering from VF [11]. The limitation of these Fourier-based analyzes is that although they can discern what frequencies occurred over the length of a signal, they cannot tell when those frequencies occurred in the signal.

Since VAs have highly time-varying spectral content, we need a method that can provide information as to the changes in frequency over time. This led to the use of more advanced time- 
frequency (TF) analysis techniques. TF analysis allows for the observation of how the spectral content of a signal is changing over time, which is useful for studying time-varying phenomena like VAs. One of the well-known TF analysis methods is time-frequency energy distributions (TFD) [12]. TFDs are used to transform a signal into an energy distribution that is a function of both time and frequency. A popular TFD used previously to analyze VAs is the wigner-ville distribution (WVD). For instance, Clayton et al. used the WVD to study the underlying processes of polymorphic VT (PVT) [13]. Patwardhan et al. also used a well-known variant of WVD (smoothed-pseudo-WVD) to extract DF from VF in order to quantify its behavior over time [4]. The issue with this type of analysis is that it was computationally expensive, and the presence of cross-term interferences in the TF plane can obscure information regarding the signal at hand.

Data driven decomposition techniques have also been used to study VAs. For instance, Tripathy et al. utilized variational mode decomposition to analyze VAs and classify them as shockable and non-shockable rhythms. Empirical mode decomposition (EMD) has also been used to classify VAs in various applications $[14,15]$. Another use of EMD was to construct the Hilbert energy spectrum (HS) from its intrinsic mode functions (IMF). The HS is capable of time-frequency analysis and has a much lower computational cost than other TFDs. It has been shown that it is possible to implement EMD with a time complexity of the same order as the Fast-Fourier-Transform (FFT) [16]. HS analysis was used previously to analyze atrial fibrillation (AF) [17].

In previous works, TF analysis was used to extract static features that quantify VF with successful results. Our group previously used wavelet-based features to classify VT from VF, and OVF from DVF accurately [8]. While this technique had quantified the organization of VF over a short window, it did not study the organization in terms of the evolution of its spectral content over time. In the real world, due to the dynamic nature of VAs and that it may cycle between different types over time, it would be useful to quantify their dynamic characteristics. The approach of using instantaneous or near real-time windowed features to quantify the progression of arrhythmias over time has not been well explored. Instantaneous TF features used previously to study VF are 
instantaneous mean frequency (IF) and instantaneous dominant frequency $[4,18,19]$. Through the use of instantaneous features, it may be possible to quantify the changes in the characteristics of VAs over time, and then classify them and/or track their progression accordingly [20].

\subsection{Motivation}

Because of the life-threatening nature of VAs, there is a great interest in discovering more effective detection and treatment methods to improve patient survival rates. Despite decades of research, there is still a high mortality rate for patients who suffer from VF. Currently available treatment options for VA patients are anti-arrhythmic drugs, controlled pacing using implantable cardioverterdefibrillators (ICD), defibrillation shocks, and ablation of affected cardiac tissues [21]. The most appropriate treatment option varies depending on the the type of arrhythmia, its progression over time, and in or 'out-of-hospital' occurrences. Thus, it is essential that a patient's arrhythmia is swiftly and accurately diagnosed.

This thesis attempts to use a signal analysis approach to characterize the dynamics of VAs in order to provide EMS (out-of-hospital scenario) and clinicians (in-hospital scenario) with better discriminatory aids to help them plan and choose the most appropriate treatment options for patients. Through the use of a computationally efficient data driven approach, the problem of accurately and swiftly diagnosing VAs may be addressed without being biased by the pre-selection of a basis function. Both instantaneous and non-instantaneous signal features will be explored in providing solutions that will address both the 'in-hospital' and 'out-of-hospital' VA scenarios. The proposed techniques could also be implemented into ICDs implanted in patients to improve the diagnostic accuracy of arrhythmias so that correct therapy options can be administered in a timely manner. Another key factor motivating this work is the access to clinical expertise in the area of VAs, which is needed to validate the proposed methodologies. Through the collaborative efforts between our research group and Toronto General Hospital, access to clinical expertise provides an ideal environment to perform this study. 


\subsubsection{Objectives}

The overarching objective of this thesis is to create methodologies that assist clinicians in selecting and modifying treatment options for patients suffering from VAs in both 'in-hospital' and 'out-ofhospital' scenarios. This main objective can be divided into 2 sub-objectives:

- Using ECG data during VA and EMD, extract signal features that capture the dynamics of VAs and use them for both long-term (in-hospital or offline) and short-term (out-of-hospital) classification of VAs.

- Using ECG data during VA and EMD, construct the EMD HS and extract TF instantaneous signal features that could be used for both long-term (in-hospital or offline) classification and near real-time (out-of-hospital) trend analysis for tracking VAs.

Each of these objectives will be addressed in the subsequent chapters 3 and 4 of this thesis. In addition, comparative analysis using existing features will also be performed. Figure 1.3 shows the detailed block diagram illustrating the proposed approaches that were performed in the study. The middle branch, which is common to both chapters illustrates how the VA ECG signals will be processed before feature extraction. The top branch shows the non-instantaneous feature extraction for classifying VAs in both 'out-of-hospital' and 'in-hospital' scenarios. The bottom branch shows how TF analysis is used to extract instantaneous features for 'out-of-hospital' VA trends analysis. Finally, both of the instantaneous and non-instantaneous features will be combined to perform a final VA classification for 'in-hospital' scenarios, shown at the end of the middle branch.

\subsection{Thesis Outline}

This thesis will be organized into the following chapters:

- Chapter 2: This chapter will present some background information of the various signal processing techniques explored in this work, as well as existing works related to these techniques. It will also discuss the pattern classification methods used, as well as the various statistical analyzes used to quantify the findings. 


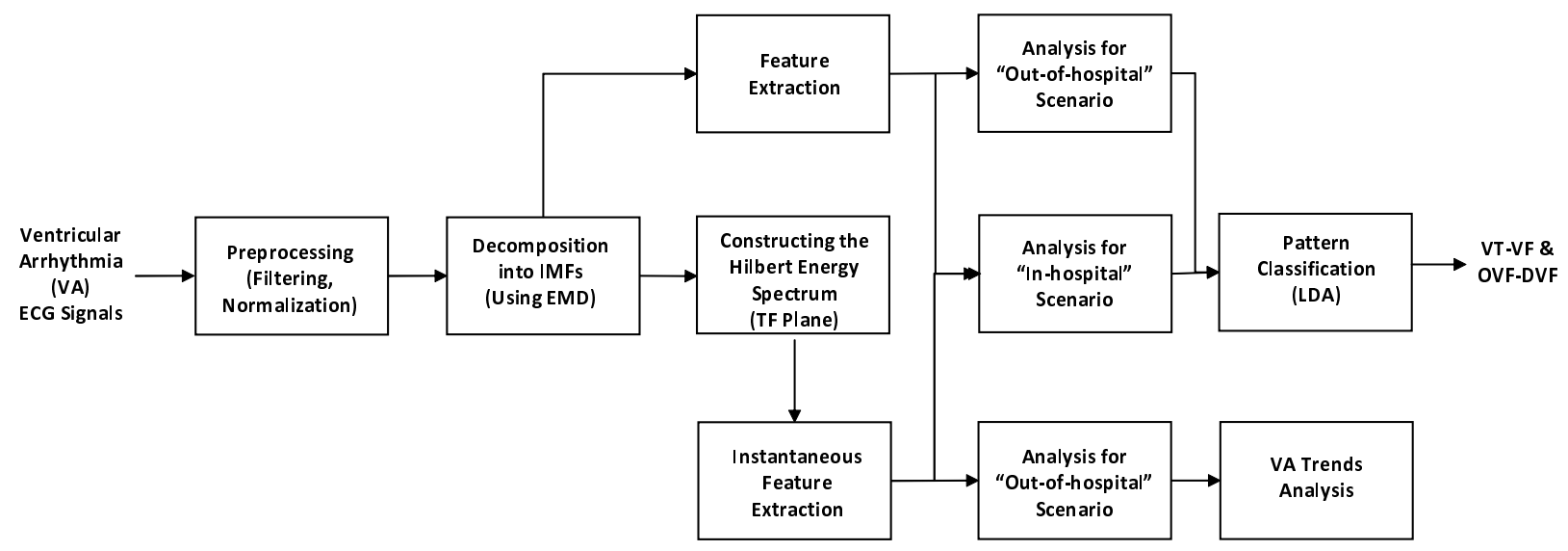

Figure 1.3: Block diagram outlining proposed approached of this study.

- Chapter 3: This chapter will present the results of performing a binary classification scheme between VT and VF, and sub-classifying VF into OVF and DVF. The first part will show the results of performing 'in-hospital' (long-term) analysis, and the second part will show the results of the 'out-of-hospital' (short-term) analysis.

- Chapter 4: This chapter will present the results of using instantaneous time-frequency features to perform 'in-hospital' (long-term) analysis to classify VAs. It will also present the result of a near real-time analysis method for detecting trends in the VAs over time. Lastly, this chapter will present the results of combining the long-term results from Chapters 3 and 4 to perform a binary classification scheme of VT, OVF, and DVF.

- Chapter 5: This chapter will bring the thesis to its conclusion by summarizing the presented findings and suggesting opportunities for future applications. 


\section{Chapter 2}

\section{Background}

7 HIS chapter will explore the various signal analysis techniques that have been used to study

1 VAs previously to provide context for this work. It will also introduce and explain the specific techniques that will be used in this thesis. The focus of this work is on the use of TF analysis and data-driven approaches, so they will be the two main branches of this chapter. There will also be some background on the pattern classification and statistical analysis methods that will be used, and to conclude a summary of the methods presented will be provided.

\subsection{Time-Frequency Analysis}

Due to the time-varying nature of VAs, the most suitable tool to analyze these signals is TF analysis methods. As we noted previously, although time-only and frequency-only methods have been used extensively in the past to study VAs, they possess limitations when studying signals with time-varying spectral content. In general, there are two types of approaches to TF analysis. The first approach uses quadratic time-frequency energy distributions (TFDs), in which a signal is transformed into an energy map that is a function of time and frequency. These distributions typically have high TF resolution, useful for visualization and extraction of complex instantaneous features, but have high computational complexity [12], [22]. However, these methods also contain cross-term artifacts in the TF plane, disturbing the interpretation of the signal at hand. The second approach is TF decomposition techniques. In these methods, a signal is approximated by a number of TF basis functions that are translated and scaled to match the structure of the signal. 
These techniques attempt to enhance certain desirable characteristics of a signal, making it suitable for classification applications [22]. However, these methods are dependent on the choice of basis function(s), and hence the modeling process will vary depending on the selection.

Due to the vast number of TF analysis methods available, we will only focus on the techniques that are fundamental to our understanding of TF analysis and those that are relevant to this thesis.

\subsubsection{Short-Time Fourier Transform}

The short-time fourier transform (STFT) is one of the earliest TF analysis techniques. Also known as the windowed Fourier transform, the STFT essentially computes the Fourier transform over short windowed segments of a signal. This is done to in an attempt to make the signal being analyzed inside the window stationary, which is fundamental to the computation of the Fourier transform. The window then shifts to the subsequent part of the signal until the signal's full length has been analyzed. The equation for the discrete-time STFT is given as [23]:

$$
S f(l, m)=\sum_{n=0}^{N-1} f(n) g(n-m) e^{\frac{-i 2 \pi l n}{N}}
$$

In Equation 2.1, $f(n)$ is the signal and $g(n)$ is the window applied to the signal. The window $g(n-m)$ is discretely shifted my $m$ points, and the energy of the signal $f(n)$ is calculated at frequency $l$ and time sample $m$. The energy of the signal is represented by the spectrogram which is calculated as $|S f(l, m)|^{2}$. The STFT also enables the user to select the type of window to use, allowing for some adaptability. For instance, adjusting the type of window used can result in different time and frequency resolutions based on its size and shape. Different windows can also affect the level of spectral and temporal smoothing. These parameters may be adapted to fit the specific characteristics of the signal to be analyzed.

The STFT has been used extensively in the past for studying cardiac arrhythmias. It was shown by Clayton et al. to be a useful tool for analyzing non-stationary signals like VF [24]. Evans et al. also used the STFT to analyze electrograms during VF in order to detect conduction blocks in 
the heart [25]. Also, Choi et al. used this method to analyze transmembrane potentials to track the spatio-temporal organization of frequencies during VF [26].

In spite of these previous works, STFT has some fundamental issues that need to be addressed. The major assumption of the STFT is that the signal being analyzed within the window is stationary. The problem is that for highly time-varying signals like VF, that assumption may not always hold true. The only way to ensure that stationarity is held is to reduce the length of window small enough so that no variations are present. However, doing that opens up another issue with the STFT, which is the tradeoff between time and frequency resolution. As we noted, the shorter the analysis window used, the more likely we are to ensure non-stationarity of the signal. Doing so also improves the time resolution of the analysis, meaning we can better localize when a frequency component occurred. However, using a shorter window conversely reduces the frequency resolution, i.e. the localization of what exact frequencies occurred. This tradeoff is due to the Heisenberg uncertainty principle [23]. Because of this inherent tradeoff, when using this method a compromise must be made between time and frequency resolution based on the spatio-temporal properties of the analyzed signal.

A potential work-around for this issue is to vary the window length depending on the frequency resolution required. Although doing so would allow for the optimization of time and frequency resolution, because of the time-varying nature of VF, the frequency is constantly changing, making this operation quite difficult. Due to theses issues, researchers have turned to alternative TF techniques that are more adaptive.

\subsubsection{Wavelet Analysis}

The wavelet transform is another tool that can be used for local time-scale decomposition. Unlike the STFT, wavelet analysis uses a dictionary of scaled and translated wavelets, or basis functions, to decompose a given signal. Each wavelet has a specific time-scale localization, and through transformation can be used to represent signal structures of vastly varying sizes [23]. The formula 
for the discrete-time version of the wavelet transform is given as [27]:

$$
W f(s, m)=\frac{1}{\sqrt{s}} \sum_{n=1}^{N} f(n) \psi^{*}\left(\frac{n-m}{s}\right)
$$

Where $s$ is the scaling factor and $m$ is the translation factor of the mother wavelet $\psi$, and $W f(s, m)$ represents the wavelet coefficients as a result of analyzing the signal $f(n)$ with the translated and scaled mother wavelet. The scale parameter $s$, which is inversely related to frequency, controls the spread of the wavelet by shrinking or dilating its time width, and the translation parameter $m$ controls the shifting in time of the center of the wavelet. Essentially, the wavelet coefficients correspond to the correlation between the signal $f(n)$ and the scaled and translated wavelet function. The scalogram represents the time-scale energy concentration of the wavelet coefficients, and is given as $|W f(s, m)|^{2}$.

There are two types of wavelet transforms implemented in discrete time. They differ based upon the discretization of the step sizes taken for the parameters $m$ and $s$. In the discrete continuous wavelet transform (CWT), the parameters should ideally be continuous, but due to computational limitations the steps are made as small as possible. However, in the discrete wavelet transform (DWT), the parameters are purposefully discretized, in order to reduce redundancy and computational complexity [23].

The wavelet transform has some advantages over the STFT that make it more suitable for the analysis of VAs. Firstly, it provides better time-scale localization of the energy in a signal. This is because the wavelet bases have a finite time support, and thus can localize a specific energy in time at a specific scale. The window length of these wavelets is also varied, allowing it to adapt to a particular signal structure. It has a larger window (scale) at low frequencies and a smaller window at high frequencies. The versatility in the selection of a basis wavelet is also an advantage over the STFT. While the STFT is limited to transforming a signal into a combination of sines and cosines, wavelet analysis has the ability to analyze a signal with any number of basis wavelets that best match the signal at hand. Additionally, the over complete wavelet transform can obtain 
a computational complexity on the same order as the FFT $\left(O\left(N \log _{2} N\right)\right)$ [23], [28], giving it the potential for real-time analysis.

Wavelet analysis has been applied extensively in the past to study VAs. One application is the classification of different types of VAs. For instance, in the review paper by Addison, Khadra et al. used scale-dependent energy content from wavelet decomposition to classify three types of arrhythmias, including VF [29]. Our group also previously used wavelet-based features to classify VT from VF, and sub-classify OVF from DVF [8]. Wavelet analysis has also been used to make predictions about resuscitation and defibrillation outcomes. Watson et al. showed the ability to detect the onset of cardiopulmonary resuscitation (CPR) in an ECG during VF [29]. In the review by Amann et al., an algorithm was developed that computed the Fourier transform of wavelet coefficients to find the maximum absolute values which were used to make decisions for defibrillation [10]. Rasooli et al. used singular value decomposition (SVD) and blind source separation (BSS) on the wavelet coefficients of pig VF data to extract independent sources related to underlying mechanisms believed to sustain VF [30]. Lastly, Endoh et al. used the mid-band energy (3-10 $\mathrm{Hz}$ ) of 1-s VF signals computed from the CWT to predict successful defibrillation [31]. Another application is the use of the DWT in the denoising of ECG arrhythmia signals before they are used to train machine learning algorithms. Acharya et al. used the DWT to denoise ECG arrhythmia signals (including VF) before using them to train a convolutional neural network (CNN) [32]. Rad et al. also used the DWT to remove undesired frequency ranges from arrhythmia signals, and then to extract features that were inputted to various machine learning algorithms for automated retrospective data analysis [33].

In spite of the improvements that wavelet analysis offers compared to the STFT, for the purposes of this thesis there remain limitations. Wavelet analysis although adaptive, is still inflexible to some extent in TF resolution due to the inverse relation between scale and frequency. Moreover it relies heavily on the pre-selection of a wavelet basis function for analysis. Additionally, proper scaling is required using the admissibility constant of specific wavelets before scalograms can be 
used to extract meaningful TF features [23]. Because there are many wavelet basis functions available, choosing one mother wavelet will inevitably bias the decomposition based upon the user's choice. That is why a data driven approach is preferred here, since it is adaptable to the inherent properties of the data it is applied on.

\subsubsection{Time-Frequency Energy Distributions}

Instead of decomposing a signal into constituent elements by a basis function, in TFD analysis a signal is transformed into an energy density map that is simultaneously a function of time and

frequency. There are many different types of TFDs, and but in this subsection we will explore only the most well-known TFDs that have been used extensively for VA analysis.

\section{Wigner-Ville Distribution}

The WVD is a TFD created by Wigner and introduced to signal processing by Ville in the 1940s [23]. Originally applied in the field of thermodynamics, the WVD is generally the Fourier transform of the auto-correlation of a signal $f(n)$ which is being lagged in both directions. Thus, it is called a quadratic or bi-linear distribution since the signal enters into the calculation twice [34]. The WVD generates a time-frequency representation (TFR) that allows for localization of the spread of energy at a particular time and frequency. The discrete version of the WVD is given as [35]:

$$
W_{x}(n, k)=\frac{N}{2} \sum_{m=-N+1}^{N-1} f(n+m) f^{*}(n-m) e^{-\frac{2 j \pi k m}{N}}
$$

Where $f(n)$ is the analyzed signal, and $W_{x}(n, k)$ is the TF energy distribution where the energy is localized at the time and frequency coordinates specified by $n$ and $k$, respectively. Note that the signal $f(n)$ used in Equation 2.3 must first be made analytic by the application of the Hilbert transform [12]. This cancels out the negative frequencies from the signal, which removes the interference between positive and negative frequencies in the TF plane [12]. Compared to other distributions, the WVD has good TF resolution. However, one of its biggest issues is the existence of cross-terms. Cross-terms are interferences that occur in the TF energy map but do not pertain to actual information about the signal at hand. They exist because of the quadratic nature of the WVD. 
When analyzing a signal that is the sum of two or more components (i.e. multi-component), these summative components are squared by the WVD, which creates additional interference terms that are not part of the original signal. The equation for this cross-term interference is shown in [34]:

$$
W(t, \omega)=W_{11}(t, \omega)+W_{22}(t, \omega)+2 \operatorname{Re}\left\{W_{12}(t, \omega)\right\}
$$

Where $2 \operatorname{Re}\left\{W_{12}(t, \omega)\right\}$ is the cross-term created by computing the WVD of a signal with 2 components $s_{1}(t)$ and $s_{2}(t)$. This problem compounds with the increasing number of components within a signal. These cross-terms may result in artifacts in the TF energy map. These artifacts can cause a misinterpretation of the energy content of a signal over time and frequency. This is especially likely when studying highly multi-component and time-varying signals like VF. This makes using the WVD a difficult proposition for this work.

In order to address the issue of cross-term interference, some variations of the WVD were developed and are discussed next. Figure 2.1 shows an illustration of how variants of WVD try to address the issue of cross-terms. However, an inevitable trade-off with TF resolution is always present.

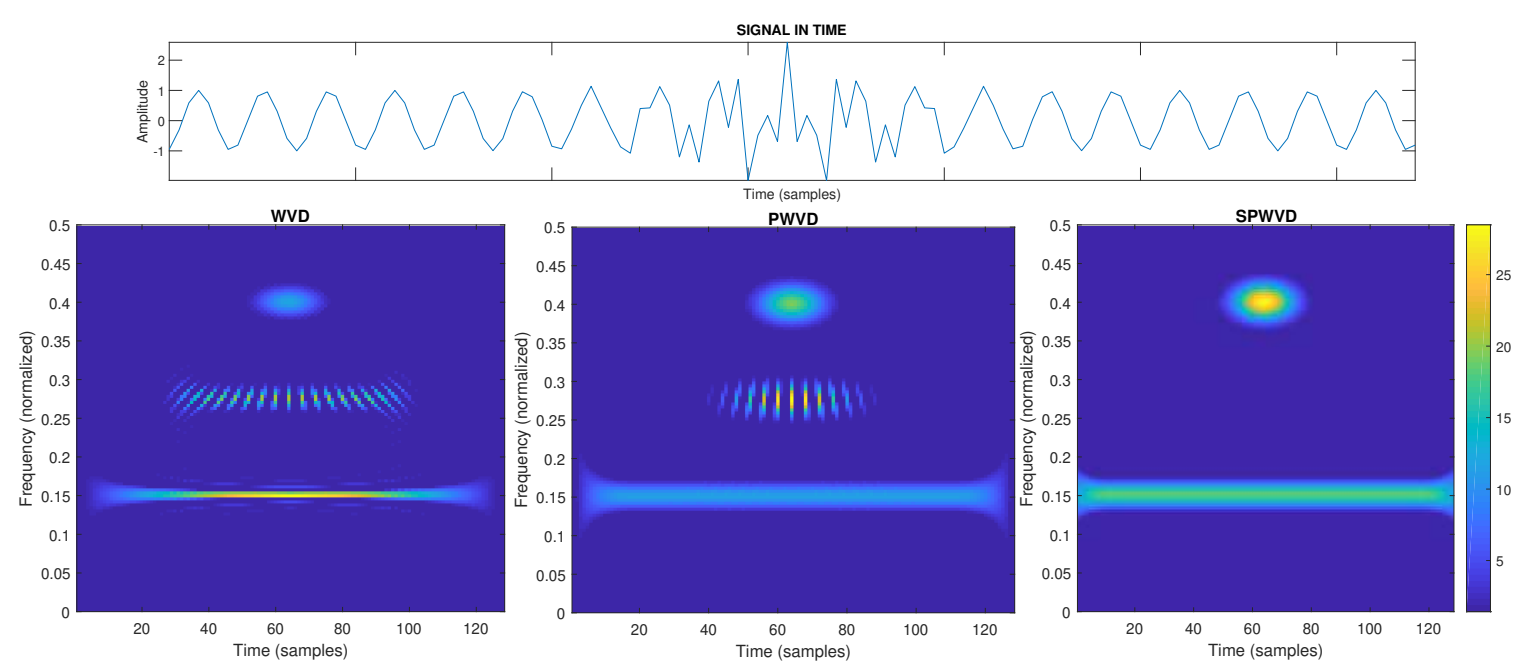

Figure 2.1: A signal composed of a Gaussian atom combined with a complex sinusoid comparing its TFR for WVD, PWVD, and SPWVD. 


\section{Variations of the WVD}

The first variation of the WVD to be discussed is called the pseudo-WVD (PWVD). The PWVD differs from the WVD by the introduction of a smoothing window that localizes the WVD around a time of interest [34]. This has the effect of reducing cross-terms in the TF plane. However, the improvements brought on by the PWVD come with tradeoffs compared to the original WVD. Firstly, many of the useful mathematical properties of the WVD are lost in the PWVD, such as satisfying the TF marginals [34]. Another detriment of the PWVD is that windowing in the time domain results in smoothing of the frequency domain which reduces the frequency resolution of the TF map. Thus, there exists an inevitable tradeoff between cross-term reduction and frequency resolution.

Another variation of the WVD is called the smoothed-pseudo-WVD (SPWVD). Similar to the PWVD, it also implements a smoothing function to reduce cross-term interference by localizing the distribution. However, unlike the PWVD which uses a simple time window, the SPWVD utilizes a separable smoothing function that allows for independent control of both time and frequency smoothing [36]. The discrete implementation of the SPWVD is given as [37]:

$$
S P W_{x}(n, k)=\frac{N_{h}}{2} \sum_{m=-N_{h}+1}^{N_{h}-1}|h(m)|^{2} \sum_{l=-N_{g}+1}^{N_{g}-1} g(l) f(n+1+m) f^{*}(n+l-m) e^{-\frac{2 j \pi k m}{N_{h}}}
$$

Where $h(m)$ and $g(l)$ represent the time and frequency smoothing functions, respectively. By manipulating the width of these two windows, it enables the user to control the time and frequency resolution of the distribution. This also creates a compromise between the TF resolution and the level or cross-terms. The more you smooth in time and/or frequency the more you reduce crossterm interference [36]. Thus, the user must decide what level of cross-term interference is tolerable based on the TF resolution required for the application.

Using the WVD and its variants to create a TFD, it is possible to extract instantaneous TF features to monitor the changes in a signal's frequency content over time. Clayton et al. used the smoothed-WVD to extract DF and mean frequency from PVT signals, and showed that DF can be 
used to predict termination of PVT. [13]. Clayton also did a comparison of different TFDs to see which one would work best for analyzing VF [24]. Patwardhan et al. used the SPWVD to study VF. In one study they extracted DF from VF electrograms, and showed an increase in DF as VF progressed [4]. They also used the SPWVD to analyze swine epicardial electrograms to quantify spatio-temporal changes during VF [18]. Umapathy et al. also used the SPWVD to extract the IF from VF signals. They used the IF in conjunction with phase maps to track rotors, one of the underlying mechanisms believed to sustain VF [19].

Although the WVD and its variants have been used to extract instantaneous TF features to track VAs, they have issues that make them unsuitable for this thesis. A major difficulty with the WVD and its variants is high computational complexity. In order to compute the TFD matrix, an FFT calculation has to be done for every instant of time. Thus, the computational cost of the WVD is of order $O\left(N^{2} \log _{2} N\right)$, where $N$ is the length of the signal [38,39]. Compared to the

FFT, the computational complexity of the WVD is greater by a factor of $N$. This means that the computational time will be $N$ times greater than the FFT. Because the purpose of this thesis is to devise methods that provide near real-time feedback to medical personnel regarding the condition of a patient's VA, using TFDs would result in wait times that are unacceptable for emergency situations. Also, the issue of cross-terms is a major concern. These artifacts in the TF plane can bias any TF features extracted from them, and spoil any physiological meaning that might be derived from its behaviour. Even if a smoothed WVD was used to reduce their effect, the cost in TF resolution would make its application infeasible for studying VAs.

\subsection{Data Driven Decomposition Techniques}

Data driven decomposition methods differ from all of the previous analysis methods we have discussed so far. Data driven techniques are not reliant on the choice of a particular basis function. This means that the decomposition is completely dictated by the inherent properties of the data itself, and so the results are not biased by operator selection. There are many types of data driven techniques, which include those used for statistical analysis, like independent component analysis 
(ICA). However, for the purposes of this thesis, we will only focus on those techniques that are computationally efficient and lend well to real-time analysis.

\subsubsection{Empirical Mode Decomposition}

Developed by Huang et al, Empirical mode decomposition (EMD) is an adaptive signal decomposition technique [40]. It is a data driven method, meaning that it is not reliant on any basis function, but rather on the inherent properties of the data itself. It is an adaptive method that allows the decomposition of non-linear and non-stationary data, like VF. EMD is also computationally efficient, making it a suitable technique for this work. EMD decomposes a signal into a finite set of IMFs. An IMF must satisfy the following two conditions: (1) the number of maxima (positive) and the number of minima (negative) must either be equal or differ by no more than one, and (2) the mean value of its two envelopes (defined by the maxima and minima) must be equal to zero [40].

The process of decomposing the data into IMFs is known as sifting. The EMD algorithm is briefly described as follows [40], [41]:

1. For a discrete-time signal $f[n]$, all of the local minima and maxima are identified.

2. The upper envelope $E_{U}$ and the lower envelope $E_{L}$ are computed by connecting the local maxima and minima, respectively.

3. The mean envelope is calculated as $E_{\mu}=\left(E_{U}+E_{L}\right) / 2$, and it is subtracted from $f[n]$ to update it as $f[n]:=f[n]-E_{\mu}$.

4. The above steps are repeated until $f[n]$ satisfies the two required conditions for an IMF, at which point it becomes the first IMF $c_{1}[n]$.

5. The first IMF $c_{1}[n]$ is subtracted from the original $f[n]$ to get the residual $r_{1}[n]$.

6. The residual $r_{1}[n]$ is now taken as the starting point, and the previous steps are repeated to find all IMFs $c_{i}[n]$ so that the final residual $r_{k}[n]$ becomes either a constant, a monotonic signal, or a signal with a single extrema where no further IMFs can be derived. 


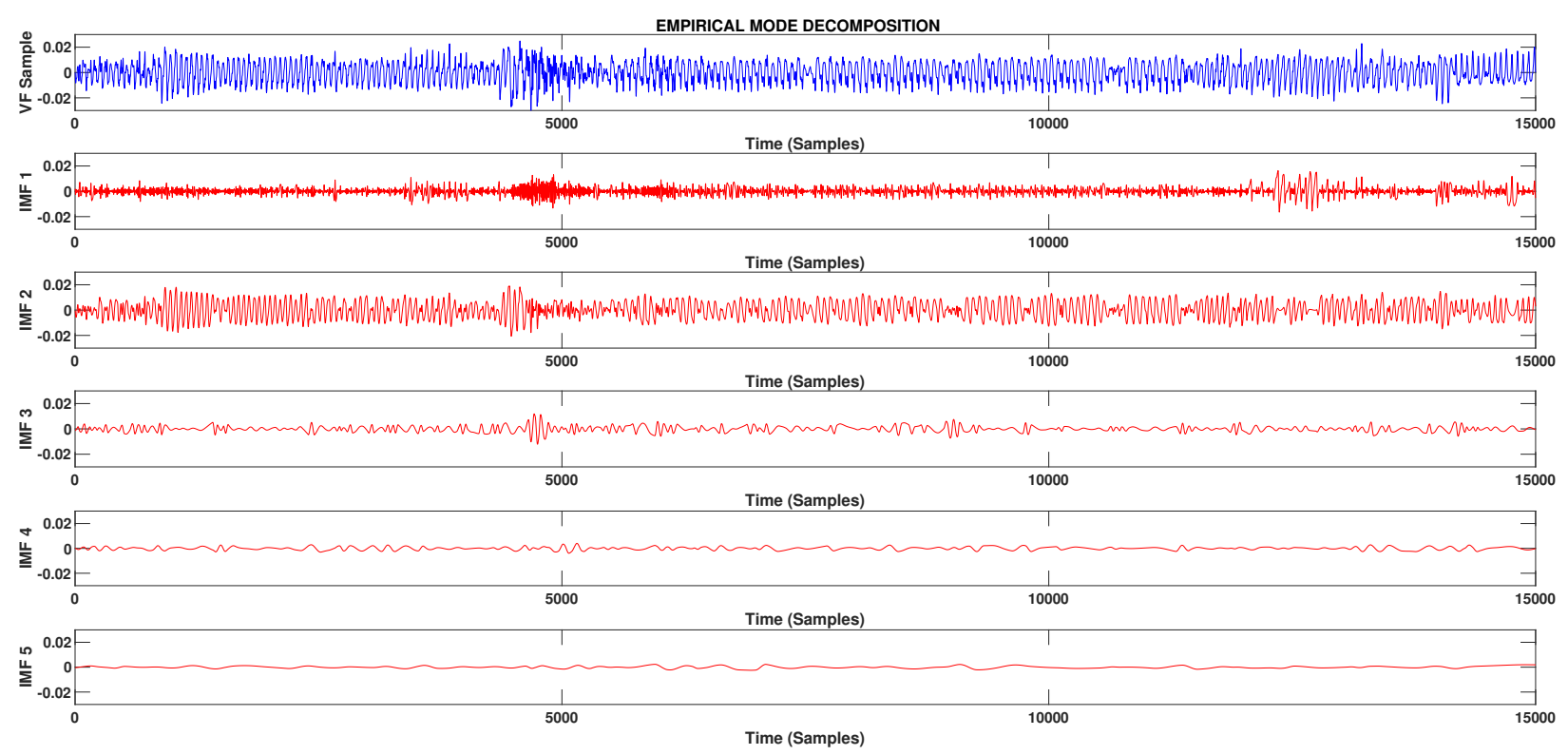

Figure 2.2: Empirical mode decomposition of a 60-s OVF sample, showing the first 5 decomposed IMFs.

The original signal $f[n]$ can be reconstructed as the sum of the $k$ IMFs and the final residual $r_{k}$ :

$$
f[n]=\sum_{i=1}^{k} c_{i}[n]+r_{k}[n]
$$

Each IMF extracted from the data represents an oscillatory mode that was imbedded in the data. Figure 2.2 shows the IMFs decomposed from a sample VF segment from the database. An IMF can contain both amplitude and frequency modulations, and can even be non-stationary so long as it satisfies the 2 criteria described above [40]. These oscillatory modes can give insight into the underlying physical phenomena that are taking place in a signal. This could prove especially useful for studying VF, as these IMFs may demonstrate some properties as to the underlying mechanisms that are sustaining VF.

Previous techniques have been used for decomposing VF signals into modal functions for the purpose of identifying underlying behaviours. In the work mentioned earlier by Rasooli et al. [30], they decomposed VF ECG signals into independent sources (IS) using BSS. These ISs are distinguished from each other based on their independence, which was ensured by ICA. Different energy and frequency-based features were then extracted from the ISs to classify successful from 
unsuccessful defibrillation cases, and showed potential to being related to underlying dynamic characteristics of VF. In the case of EMD, these IMFs extracted from VF may also show some relation to the dynamic processes that maintain VF.

EMD has some strong benefits compared to other methods for application to this work:

1. Unlike previous methods, EMD is fully data-driven and the IMFs are extracted based on the intrinsic properties of the data itself. The IMFs may reveal hidden oscillatory behaviours that could be related to the physical phenomena taking place in the heart during VF.

2. Another strong benefit of EMD is the ability to construct the HS from its IMFs. More details on the construction and meaning of the HS will be given later. The important aspect of the HS as it pertains to this thesis is that since it is a TFR, it is possible to extract instantaneous TF features from it. These instantaneous features could be used to track the changes occurring in the spectral and energy content of VF over time. Similar to TFDs, this EMD-based approach has the ability to monitor the evolution of VAs over time.

3. An additional benefit of the EMD algorithm is its computational complexity. Unlike TFDs that are considerably more computationally costly, EMD may be implemented with similar computational complexity as the FFT [16]. Thus, EMD-based algorithms are more suitable for use in real-time analysis. This means that the EMD-HS could be used to monitor the progression of ventricular arrhythmias in 'out-of-hospital' emergency situations and provide immediate feedback to EMS personnel.

One of the fundamental concerns with EMD that must be discussed is mode mixing. Mode mixing is present when an IMF consists of two or more oscillations of vastly different scales (frequencies), or when a similar scale exists in multiple different IMFs [42]. This is often the result of signal intermittency, in which a signal's frequency components are abruptly stopping or changing. The problems that mode mixing causes are that, firstly, it obscures the physical meaning of individual IMFs since the oscillatory modes that could represent physiological mechanisms are overlapped and interspersed. Also, mode mixing can cause aliasing in the TF map, as the smooth transitions 
from one scale to another are lost [42]. All of this makes it more difficult to interpret the results and draw conclusions about the data being analyzed. In order to address mode mixing, a number of updated EMD algorithms have been developed. However, for the purposes of this thesis, EMD is still the most suitable approach. The biggest challenge with mode mixing is that is obscures the physiological meaning of individual IMFs by blending oscillatory patterns together. However, the focus of this work was on relative feature extraction among sets of IMFs and instantaneous features extracted from the HS TFRs for VA classification. The features extracted were thus meant to highlight relative differences between different groups of VAs for discrimination, and were not focused on decoding meaningful mechanistic insights regarding VF. In addition, maintaining low computational cost was essential to the real-time application of the proposed methodology for use in emergency settings. Noise assisted EMD methods are significantly slower because entire epochs of IMF realizations need to be generated before the final IMFs are computed (explained later). Due to the time critical nature of studying VAs, using noise assisted EMD method would be computationally prohibitive to the fundamental goals of this work. For these reasons, EMD was the most appropriate choice for this thesis.

EMD-based analysis methods have been used previously in many biomedical applications, including for classifying VAs. A group of highlighted works relevant to this thesis is described here. Huang et al. [17] used the HS to study AF signals. The IF distribution was calculated by computing a weighted average of the instantaneous frequencies of the individual IMFs, and from the IF distribution features were extracted to successfully discriminate self-terminating from nonterminating AF [17]. Arafat et al. used an EMD approach to classify NSR from VF using the orthogonality of the first 3 consecutive IMFs with a high degree of accuracy [14]. Bai et al. used an EMD based method to discriminate NSR, VT and VF. They extracted spectral entropy and the energy rate (relative to total energy) of the first IMF only, and used a Bayes classifier to classify the 3 rhythms [15]. Kaleem et al. used EMD to extract a set of energy, temporal and spectral based features from IMFs to classify pathological speech from normal speech [41]. EMD has also been used extensively in electroencephalogram (EEG) analysis, especially for epilepsy detection. They 
often use the HS to track instantaneous frequencies and amplitudes. A review of EEG analysis works may be found here [43].

\subsubsection{Additional EMD-based Methods}

Ensemble Empirical Mode Decomposition (EEMD) is an updated EMD algorithm developed to address the key problem of mode mixing in the IMFs [42]. EEMD resolves this problem by computing the IMFs over a large number of realizations of the same signal with different amounts of finite Gaussian white noise added to it. The ensemble average of each of the IMFs is taken, and those are treated as the 'true' IMFs [42]. Adding the white noise ensures that no scales are missing from the signal's spectrum. EEMD is essentially a noise-assisted data analysis method. The white noise is cancelled out from the final IMFs when they are averaged together. The algorithm for EEMD is described as follows [44]:

1. Let $f[n]$ be the input signal, then $f^{i}[n]=f[n]+w^{i}[n]$, where $w^{i}[n]$ is the $i^{t h}$ realization $(i=1, \ldots, I)$ of Gaussian white noise.

2. Each $f^{i}[n]$ is decomposed by EMD to produce the modes $I M F_{k}^{i}[n]$ where $k=1, \ldots, K$ indicates each mode (see EMD algorithm above).

3. Compute $\overline{I M F_{k}}[n]=\frac{1}{I} \sum_{i=1}^{I} I M F_{k}^{i}[n]$, where $\overline{I M F_{k}}[n]$ is assigned as the 'true' IMF of the $k^{t h}$ mode of $f[n]$ corresponding to the ensemble average of all $I M F_{k}^{i}$.

By adding Gaussian white noise to the signal, it provides a uniform reference scale distribution from which to extract the IMFs. This prevents there from being disparate scales by populating the entire TF plane uniformly, and effectively solving the problem of mode mixing [42].

Despite the improvements that EEMD makes over EMD, there are some new issues created. Firstly, because the IMFs are an amalgamation of a whole set of different EMD decompositions, it is not possible to exactly reconstruct the original signal. Another issue is that variations in the amounts of white noise added could alter the IMF decomposition, and so it is no longer a fully adaptive technique. The main complication with EEMD in regards to this thesis is the increase in computational time. In order to accurately determine the true IMFs, a large number of realizations 
is required so that the white noise if effectively removed from the final ensemble average. These $I$ realizations require that the original EMD algorithm be repeated $I$ times, essentially increasing the computational time of EEMD by a factor of $I$. This drastic increase in the computational time of EEMD makes it less suitable for online analysis compared to EMD. The objectives of this thesis require a methodology that is fast enough to be used in real-time emergency settings, and EEMD does not satisfy this requirement.

In addition to EEMD, there have been other recent developments to the EMD algorithm to address other issues. One of the main problems with EEMD is the residual noise left over in the IMFs after the ensemble averaging. An algorithm developed by Yeh et al. (2010) called complementary ensemble EMD (CEEMD) was developed to deal with this issue [45]. CEEMD works by generating two sets of IMF ensembles, one with positive and one with negative noise added to the signal. When the ensemble average of these two sets of IMFs is computed, the white noise is more effectively cancelled out and the residual noise level is significantly reduced compared to EEMD [45]. Although the resulting IMFs are very similar to EEMD, with CEEMD the signal reconstruction is more accurate as the residue noise is greatly reduced. However, signal reconstruction from the IMFs is also possible using original EMD. Since the computation of an ensemble of IMFs is still required, the computational cost is still significantly higher than EMD.

Another updated EMD algorithm is the complete ensemble EMD with adaptive noise (CEEMDAN). Created by Torres et al. (2011) to deal with the issues of EEMD, the CEEMDAN algorithm differs from EEMD in a few ways. The first IMF is computed the same way as in EEMD, and the first IMF is subtracted from the original signal to obtain the first residue, $r_{1}(n)$. However, white noise is then added directly to the residue from here, and an ensemble of EMD decompositions is done to obtain the first mode of $r_{1}(n)$. The second IMF is then the ensemble average of these first modes. The second residue is calculated by subtracting the second IMF from the first residue, and this process is repeated until all of the possible IMFs are extracted (see [44] for the step-by-step algorithm). Another benefit of CEEMDAN over EEMD is that is provides an exact reconstruction 
of the original data from the IMFs. Because CEEMDAN guarantees a complete decomposition, it requires a smaller ensemble size than EEMD, which does reduce the computational cost. Despite these improvements, the computational speed of EMD cannot be exceeded by these noise averaged algorithms, and so EMD remains the best choice for the real-time analysis proposed in this thesis.

Lastly, a recent mode decomposition method worth mentioning is called Variational Mode Decomposition (VMD). It is a decomposition algorithm developed by Dragomiretskiy and Zasso (2014) [46]. Based on Wiener filtering, the VMD algorithm was designed to address the sensitivity to noise and sampling present in EMD. VMD decomposes data into a finite set of modes based on the property of narrow bandwidth. The center frequency is used as the starting point for decomposition. Both the modes and center frequencies are extracted by solving the variational optimization problem [47]. VMD has a few advantages over EMD, in that its modes are extracted independently and non-recursively from one another. It was also shown in some simple examples to be more noise robust and better able to separate tones than EMD [46]. Some drawbacks of VMD are that it does not directly deal with global trends within a signal, and it is not suitable for analyzing long-duration non-stationary signals without segmenting them. It also requires the user to select the number of modes prior to decomposition [46]. All of these factors make VMD a less adaptive technique than EMD, and less suitable for analyzing non-stationary signals like VF.

\subsubsection{Hilbert Energy Spectrum and TFR}

The IMFs extracted from EMD can be used to construct a TFR via the HS. Combining the use of EMD and the HS is also known as the Hilbert-Huang Transform (HHT) [48]. The HS is an energy density distribution that is a function of both time and frequency. The HS is defined by the following equation $[48,49]$ :

$$
\begin{gathered}
H S_{j}(\omega, n)= \begin{cases}a_{j}^{2}(n), & \omega=\omega_{j}(n) \\
0, & \text { otherwise }\end{cases} \\
H S(\omega, n)=\sum_{j=1}^{k} H S_{j}(\omega, n)
\end{gathered}
$$


Where $a_{j}(n)$ and $\omega_{j}(n)$ are the instantaneous amplitude and instantaneous frequency of the $j^{\text {th }}$ IMF, respectively. Note that the IMFs are made analytic by the Hilbert transform prior to the HS calculation. Another important thing to note is that the instantaneous amplitude $a_{j}^{2}(n)$ is squared in the above equation, which makes it an energy spectrum as opposed to an amplitude spectrum [48]. An example of the HS extracted from a VF sample is shown in Figure 2.3. More synthetic signal examples are provided in Appendix A, Figure A.1. The HS has some properties that are superior over other TFRs. For instance, the TF space is divided into equal-sized bins specified by $\Delta t \times \Delta \omega$. The $\Delta t$ can be as small as the sampling rate step, and $\Delta \omega$ can assume any value on a continuous scale up to the Nyquist frequency [48]. So, the TF resolution can be controlled more precisely than any Fourier-based methods. Due to this capability, the HS is able to produce better TF resolutions compared to traditional TFDs. From the HS, instantaneous features may be extracted from its TF plane, like IF. However, as Huang explains in [48], the HS has a major advantage in defining the IF compared to the WVD. When observing the equation for the WVD, it has a striking similarity to the Fourier power spectrum with a slight difference in shifting. It is commonly known that Fourier analysis is unsuitable for non-stationary signals due its inability to resolve time variation, and so the physical meaning of the IF extracted from WVD is questionable [48]. The IF extracted from the WVD would then simply be the average of all frequencies at a particular time, with no detailed information about specific frequencies present.

As we saw in the previous section on EMD, there have been a number of works published that used Hilbert spectral analysis to study biomedical signals [17], [41]. To the author's knowledge, no works have been published that demonstrate the use of EMD-HS instantaneous features to study VAs over time. By utilizing the HS, instantaneous TF features can be extracted that are capable of tracking the progression of time-varying spectral content of VAs. This could be helpful in identifying particular arrhythmias, as well as detecting when an arrhythmia has evolved into another type. 

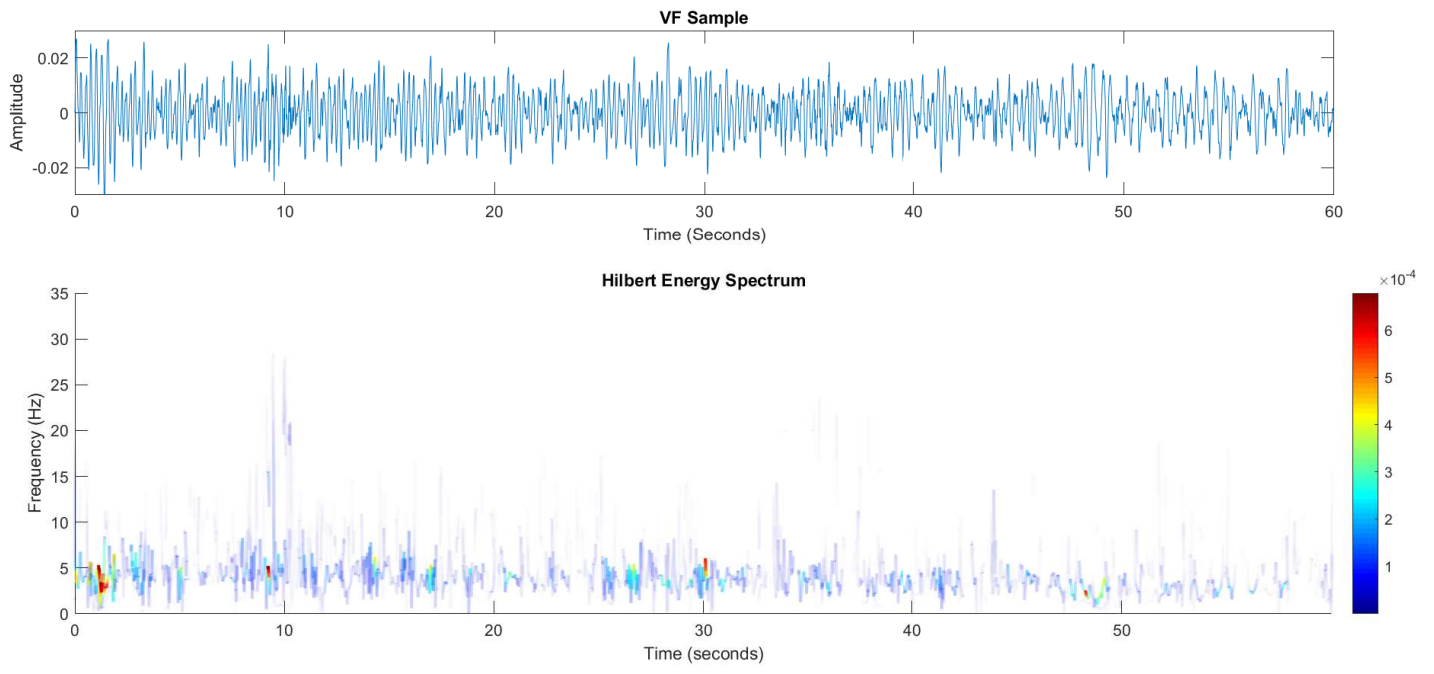

Figure 2.3: The plot of a VF sample with its Hilbert Energy Spectrum below it.

\subsubsection{Instantaneous Features}

The ability to observe the changes in the quantity of a particular feature over time adds another dimension to the analysis of time-series data. Instantaneous features as defined in this context are quantities related to signal characteristics that are also a function of time. This means that it is possible to observe how a quantity is changing over the time span of a signal. In this section, we will limit ourselves to instantaneous features that have proven successful previously at characterizing biomedical signals, or that show promise to do so. We will start with the TF instantaneous features that can be extracted from TFRs.

\section{Instantaneous Time-Frequency Features}

Instantaneous TF features can be extracted from TFRs like the HS. One method of extraction is by the moment method. The time-moments of a TFR generate spectral based features that are functions of time. One of the most useful instantaneous features that is derived in this manner is the IF [50,51]. The IF is known as the first time-moment of a TFR. The discrete-time equation for calculating the IF is given as $[19,52]$ :

$$
I F(n)=\frac{\sum_{\omega} \omega H S(\omega, n)}{\sum_{\omega} H S(\omega, n)}
$$


In which $H(\omega, n)$ is the TFR analyzed, and $\omega$ is the index for the frequency range. The IF can be thought of as the mean frequency of the signal at every time instant. Sometimes referred to as the center of gravity in frequency [50], it represents the general trend of frequency over time. The IF has been proven useful for studying VF, because it can capture the changes happening in the timevarying spectral content of VF over time. These works that were discussed previously used IF to study VAs $[4,18,19]$. The information gleaned from IF could be used to identify and discriminate types of VAs, as well to track the progression of VAs to monitor when a transition occurs from one VA to another.

Another instantaneous TF feature that can be derived by the moments of a TFR is the squared instantaneous bandwidth $\left(I B^{2}\right)$. The $I B^{2}$ is based on the second conditional time-moment of the TFR, and its discrete equation is defined below [50,51,53]:

$$
I B^{2}(n)=\frac{\sum_{\omega} \omega^{2} H S(\omega, n)}{\sum_{\omega} H S(\omega, n)}-I F(n)^{2}
$$

$I B^{2}$ is a bandwidth parameter that quantifies the spread of frequencies at each time. The $I B^{2}$ feature can give insight into how the temporal complexity of a signal is changing over time. The more complex a signal is, the higher the bandwidth tends to be. As the complexity of VF tends to change over time, this $I B^{2}$ parameter could be promising for characterizing VAs and their dynamic behaviour over time.

\section{Instantaneous Energy}

Instantaneous energy (IE) is another simple time dependent feature that could provide important information about the changes in a signal over time. The calculation of IE is simply the square of the magnitude of the signal [54]. The formula for calculating IE for a signal $f(n)$ can be defined as:

$$
I E(n)=|f(n)|^{2}
$$

By monitoring the energy at every time instant, the IE gives an indication as to how the energy content of a signal in changing over time. IE has been used in the past for biomedical signal 

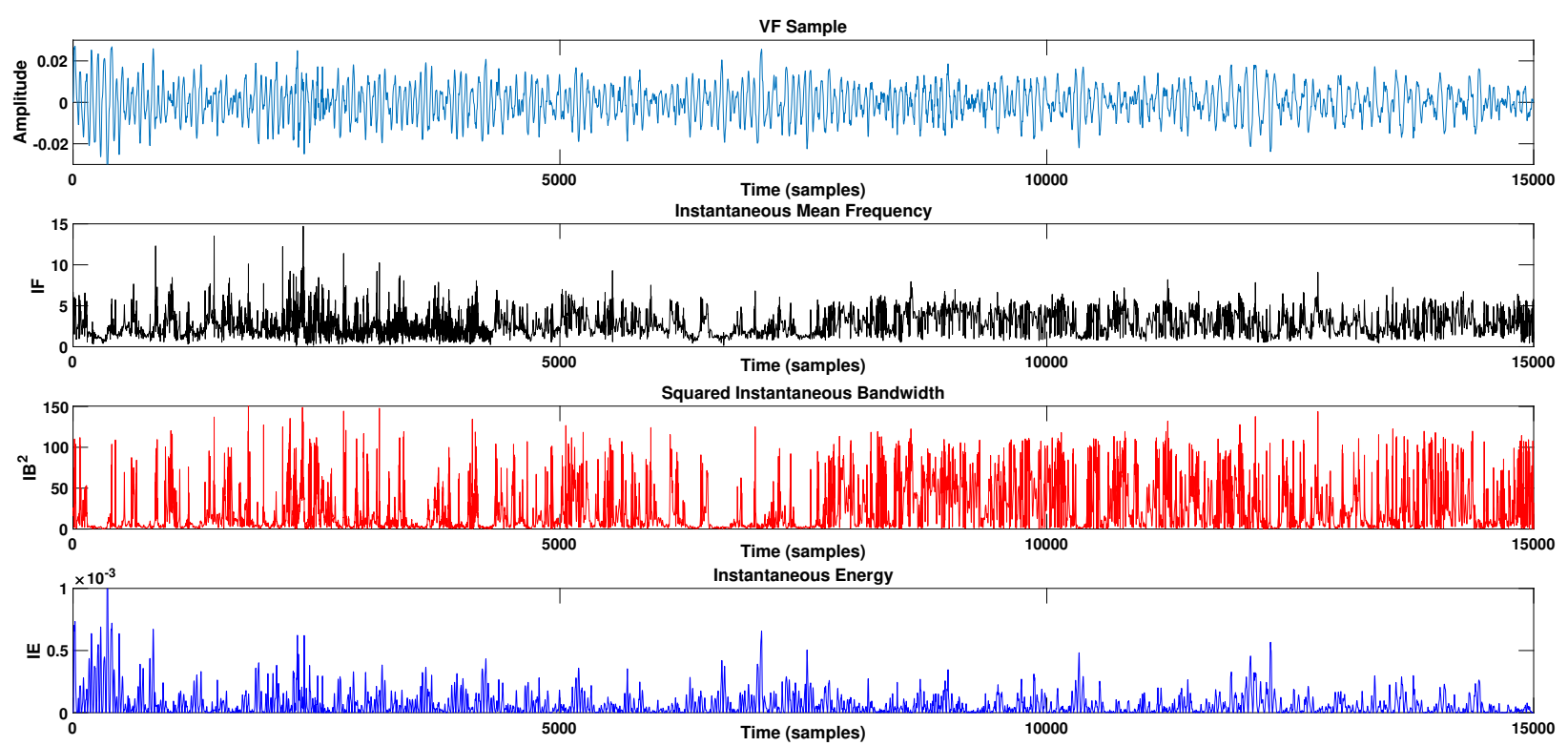

Figure 2.4: The plot of a VF sample with along with its 3 instantaneous features: IF, $I B^{2}$, and IE.

applications. It was used in heart sound segmentation to detect sounds ' $\mathrm{S} 1$ and 'S2' by capturing the energy of ECG signals [54]. IE has also been used to classify sleep stages by measuring EEG band energies [55]. The insight gained from IE previously lends to the belief that it could also be useful for studying VAs. As VF progresses, the condition of the heart tends to worsen from a lack of coronary circulation, and this may be reflected in the ECG. The IE feature would be capable of capturing this time-varying behaviour in VF. Fluctuations in the IE over time could also be indicative of complexity within the ECG signal, which may help with arrhythmia discrimination. Figure 2.4 illustrates the IF, $I B^{2}$, and IE extracted from a VF ECG sample.

\subsection{Pattern Classification and Statistical Analysis}

In order to use a set of features to separate groups of data, we need to implement a pattern classifier. A pattern classifier needs to create the best discrimination between groups of interest. Doing so will allow the system to make accurate decisions regarding how to sort new instances of information. This is especially crucial when studying VAs. As discussed earlier, in many previous studies involving VAs a set of common features is extracted from all of the signals, and they are used to train a pattern classifier that segregates the VAs into 2 or more groups (i.e. NSR, VT, 
VF). Accurately discriminating the different arrhythmias is essential in helping medical personnel choose the correct treatment option to administer to a patient. Classification accuracies are assessments used to quantify the performance of a pattern classifier. They can also compare the performance of different features used to perform the same task. In this section we will discuss the well-known pattern classification method used in this thesis called Fisher's Linear Discriminant Analysis (LDA) [56]. We will also touch upon how we will assess the performance of this classifier, as well as some statistical approaches that we will use to quantify the the results of our analysis.

\subsubsection{Fisher's Linear Discriminant Analysis}

LDA is a machine learning algorithm that creates linear boundaries for separating classes of data [56]. It is a supervised approach, meaning that it is initially trained with data in which the correct class labels are a priori knowledge. For a biomedical application, these data are pre-labeled by experts in the medical field. It is the role of the LDA classifier to discriminate new instances of data based upon the characteristics that will highlight the differences that the clinical experts used to label the training data.

Since LDA is only capable of creating linear boundaries for separating groups of data, the features used must be strong discriminators in order for classification to be successful. If the features are not highly discriminant, then LDA will perform poorly at classifying the data. Thus, it is es-

sential that the features used to train the system are meaningful and powerfully discriminant. This way, the focus of analysis is less on the machine learning approach, and more on the usefulness of the features extracted from the data.

When attempting to separate multiple groups $c$, the number of linear discriminant functions required is $(c-1)$. Visually, one can picture a single line separating 2 groups, 2 lines separating 3 groups, etc. Multiple discriminant functions will eventually separate the feature space into regions, where each class is assigned to a region. However, it is possible for a region to be ambiguous and 
belong to no class. LDA can be thought of as projecting $x$, a $d$-dimensional feature set, onto a new feature set $y$, by scaling it with weight vector matrix $w$. The matrix $\mathbf{W}$ is the matrix containing all of the weight vector matrices $w_{i}$ for each class, with a size of $d \times(c-1)$. The projection of $x$ onto $y$ is given as [56]:

$$
\mathbf{y}=\mathbf{W}^{\mathbf{t}} \mathbf{x}
$$

To find the optimal weight vectors $\mathbf{W}$, the criterion function $\mathbf{J}(\mathbf{W})$ is used. The equation for $\mathbf{J}(\mathbf{W})$ is given below. Within its calculation are two parameters, the between-scatter matrix $S_{B}$ and the within-scatter matrix $S_{W}$. These matrices provide information as to the amount of variability between the classes themselves and between the samples within each class, respectively. The equations for $S_{B}$ and $S_{W}$ are shown below.

$$
\begin{gathered}
J(W)=\frac{\left|W^{t} S_{B} W\right|}{\left|W^{t} S_{W} W\right|} \\
S_{B}=\sum_{i=1}^{c} n_{i}\left(m_{i}-m\right)\left(m_{i}-m\right)^{t} \\
S_{W}=\sum_{i=1}^{c}\left(\sum_{x \in D_{i}}\left(x-m_{i}\right)\left(x-m_{i}\right)^{t}\right)
\end{gathered}
$$

In Equations 2.14 and 2.15, $n_{i}$ is the number of samples in class $i, m_{i}$ is the mean for each class, and $m$ is the total mean of all mean values $m_{i}$. The goal is to maximize the value of $\mathbf{J}(\mathbf{W})$ in order to optimize the weight vectors. When the weight vectors are optimized, the inter-class separation will be maximized, and the intra-class variance will be minimized. The weight vectors thus provide the best projection of $x$ onto $y$ and form the linear boundaries of the LDA classifier.

The LDA classifier is trained to produce the optimal boundary based on the training set that was used. However, this does not necessarily mean that the boundary formed will produce the best classification result for the testing set. In order to remove bias created by the selection of a training and testing set, cross-validation is needed to generalize the results. 


\subsubsection{Cross-Validation}

Cross-validation (CV) is the process of dividing the $n$ samples of your dataset into $m$ equally sized sets of data. The training process is done using all but one of these $m$ sets, and the remaining set is used to test the classifier. This procedure is repeated $m$ times until all of the sets have been tested on. CV is a heuristic approach, and implementing it does not guarantee improved results. The number of sets and samples within each set may be experimented with. The validation error can be calculated to estimate the accuracy using a given number of sets [56].

The extreme form of $\mathrm{CV}$ is known as the leave-one-out method (LOO). In LOO, a single sample is left out and the classifier is trained with all of the remaining samples. If there were $n$ samples, then LOO would train with $n-1$ samples and test with the one remaining. This procedure is repeated $n$ times for all samples. The classification accuracy is assessed by the testing results of each sample. The leave-one-out mean is computed as [56]:

$$
\mu_{i}=\frac{1}{n-1} \sum_{j \neq i}^{n} x_{j}
$$

This is the sample average of the dataset if the $i^{\text {th }}$ sample is left out. The Jackknife estimate is the mean of the leave-one-out means, and its formula is:

$$
\mu_{(.)}=\frac{1}{n} \sum_{i=1}^{n} \mu_{(i)}
$$

LOO ensures that the testing results are as generalized as possible, and is especially useful for assessing small datasets.

\subsubsection{Classification Accuracy}

Classification accuracy is used to assess the performance of a classifier trained with a certain set of features. The equation for classification accuracy is [57]:

$$
\text { Accuracy }=\frac{T P+T N}{T P+F P+T N+F N}
$$

Where $T P$ stands for True Positive, $T N$ for True Negative, $F P$ for False Positive, and $F N$ for False Negative. In the example of classifying healthy and sick patients, a $T P$ would be a patient 
correctly diagnosed as being sick. A $T N$ would be a healthy patient correctly diagnosed as a healthy patient. A FP would indicate a healthy patient who was misclassified as a sick patient, and a $F N$ would be a sick patient who was misdiagnosed as a healthy patient. Using these 4 categories, the sensitivity and specificity of a classifier is defined as [57]:

$$
\begin{aligned}
& \text { Sensitivity }=\frac{T P}{T P+F N} \\
& \text { Specificity }=\frac{T N}{T N+F P}
\end{aligned}
$$

These two values give more insight into the performance of the classifier at discriminating each group individually. This is a useful visualization in cases where it is more crucial to detect one group over another. When combined together they produce the overall classification accuracy.

\subsubsection{Receiver Operating Characteristic}

The Receiver Operating Characteristic (ROC) is a quantifiable method for analysis used to evaluate the effectiveness of a pattern classifier. They illustrate how a change in the decision boundary between classes affects the specificity and sensitivity. The sensitivity or TP rate is plotted on the vertical axis while (1-specificity) or FP rate is plotted on the horizontal axis [57]. The diagonal line $y=x$ bisecting the ROC space represents a random classifier that assigns a label arbitrarily. A classifier that occupies a point in ROC space above the diagonal line performs better than random [57]. An ROC curve is generated by moving the threshold or boundary and recalculating the TP and FP rates of the classifier, plotting the new point in ROC space each time. The higher above and to the left an ROC curve lies relative to the diagonal line, the better that classifier's performance is. A numerical way of comparing ROC curves is by the 'area under the curve' (AUC). The AUC is a scalar value that theoretically always lies between 0.5 and 1 , and it provides a simple way of quantifying the ROC performance [57]. The higher the AUC is, the more robust the classifier is at discriminating the groups. The AUC proves quite useful for comparing classifiers, especially when evaluating specific features used for a classification task. A higher AUC value will indicate that one feature performs better than another, and helps determine which features to use for a given classification problem. The AUC could also evaluate a classifier that uses multiple features 
together, allowing the user to explore the effectiveness of various combinations of features to see which is the most robust.

\subsection{Chapter Summary}

This chapter provided an overview of the different TF analysis techniques explored for use in this thesis. It began by reviewing the STFT and its drawbacks, followed by Wavelet analysis. We then explored TFDs, specifically the WVD and its variations, along with a few other notable distributions. Despite their ability to produce TFRs, their computational complexity proved undesirable for real-time applications. Data-driven techniques were investigated, particularly EMD and its updated algorithms. EMD proved to be the best suited for its low computational cost and TF analysis derived from the HS. We then discussed the HS and its computation, as well as some instantaneous features suited for tracking VAs. Lastly, we reviewed our pattern classification method of LDA and leave-one-out cross-validation (LOOCV), and some statistical and performance measures for assessing the performance of the classifier. 


\section{Chapter 3}

\section{EMD-IMF Energy Features for VA Classification}

$\mathrm{T}$

WE optimal treatment options for patients suffering from VAs varies from one type of arrhythmia to another. It is crucial that a patient's VA is accurately diagnosed so that the most appropriate treatment is administered. It is also important that this diagnosis is done swiftly, especially for VF patients where the survival window is a matter of minutes. As shown in the literature review, many works have been done to classify VT from VF. The more difficult problem lies in accurately detecting different types of VF, such as OVF and DVF. Detecting these VF subclasses will give insight to medical personnel as to the level of organization, response to therapy, and severity of the VA which may assist them in optimizing resuscitation. This methodology could also be used to improve ICD arrhythmia classification, optimizing the selection of therapy options while implanted in patients. This chapter will present the results for two types of VA analysis: long-term (in-hospital) and short-term (out-of-hospital). It will be using non-instantaneous EMD features extracted directly from IMFs for quantifying dynamic VA changes over time. In the longterm analysis, we will analyze the VAs over their full duration in order to identify dynamic markers that may assist doctors or clinicians in diagnosing the VAs in an offline setting. In short-term analysis, we will extract features over short windows and perform cumulative averaging to monitor the progression of the VAs over time. This feedback may be used by EMS personnel to track the progression of VAs, or monitor the efficacy of therapies on patients to assist them in making appropriate adjustments, accordingly. The proposed work presented in this chapter is highlighted in 


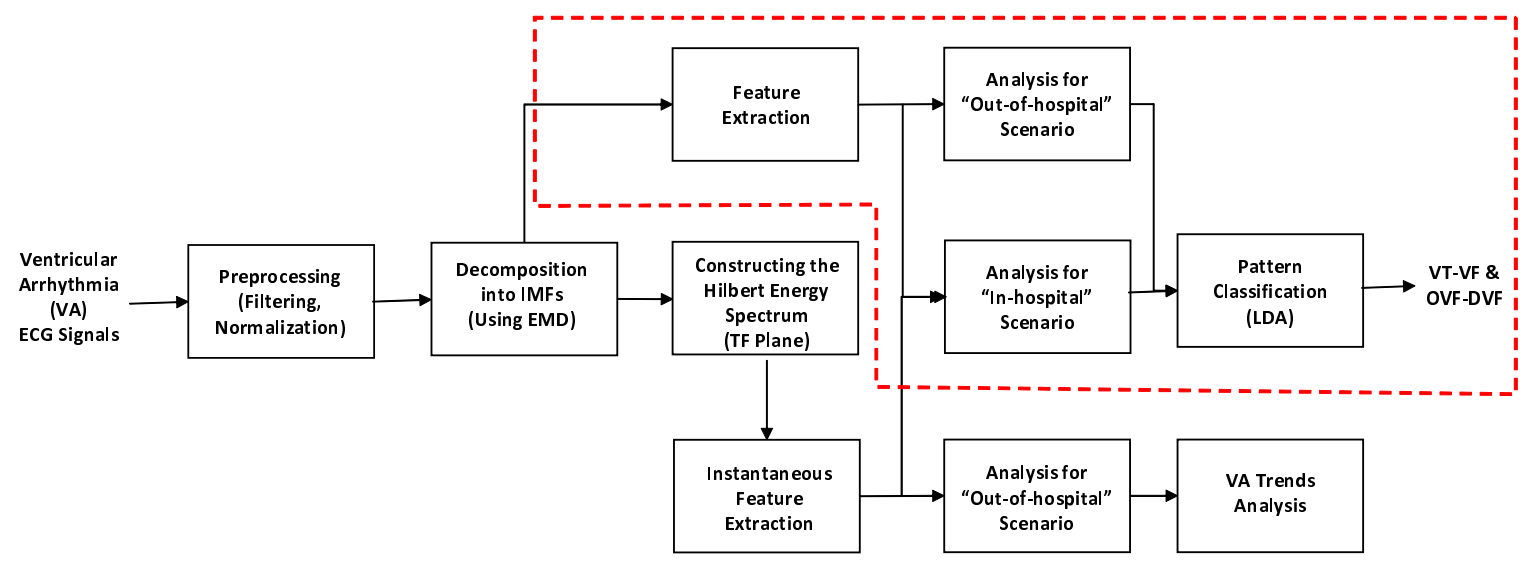

Figure 3.1: Block diagram highlighting the proposed work discussed in this chapter.

the block diagram in Figure 3.1.

\subsection{Methodology}

This section will present the entire procedure used to acquire the results of this chapter. It will start with an an overview of the the ECG database that was used. The steps for the EMD method used for extracting the IMFs will then be explained. Following that a discussion of the feature extraction will be presented along with the equations and methods used to acquire the features. This section will close with an explanation of the pattern classification techniques to be used to test the results of this proposed approach.

\subsubsection{ECG Database \& Pre-processing}

The database used for this study consisted of 61 ECG segments of 3 different types of VAs: 22 VT, 17 OVF, and 22 DVF segments. They were extracted from the MIT-BIH ventricular arrhythmia database and the Creighton University ventricular tachyarrhythmia database [58]. Each of these segments is 60-s in length and were originally sampled at $250 \mathrm{~Hz}$ (i.e, 15000 time samples each). We choose longer VA segments to include the natural variations and compositions that evolve over time to mimic the real-world scenario to track dynamic VA changes, unlike previous works that decided on the type of VA by observing shorter windows. The arrhythmia labels for these signals 
were verified by our clinical collaborators from Toronto General Hospital, University Health Network. Due to the length of these ECG segments and the natural variations that exist over time, they were labeled as 'mostly' VT or 'mostly' OVF or 'mostly' DVF for the purpose of this work. This is highly relevant in a real-world scenario as VA evolves over time and hence labeling/tracking them on a cumulative basis over time would be ideal.

Prior to analysis, each of these ECG signals were band-pass filtered between $0.3 \mathrm{~Hz}$ and 30 $\mathrm{Hz}$ to remove high frequency and low frequency artifacts. They were also energy normalized to ensure that all of the signals maintained the same cumulative energy. This was done to remove any potential bias caused by the absolute amplitude of the signals. Figure 3.2 below illustrates ECG samples for each of the 3 different VA types in this database.
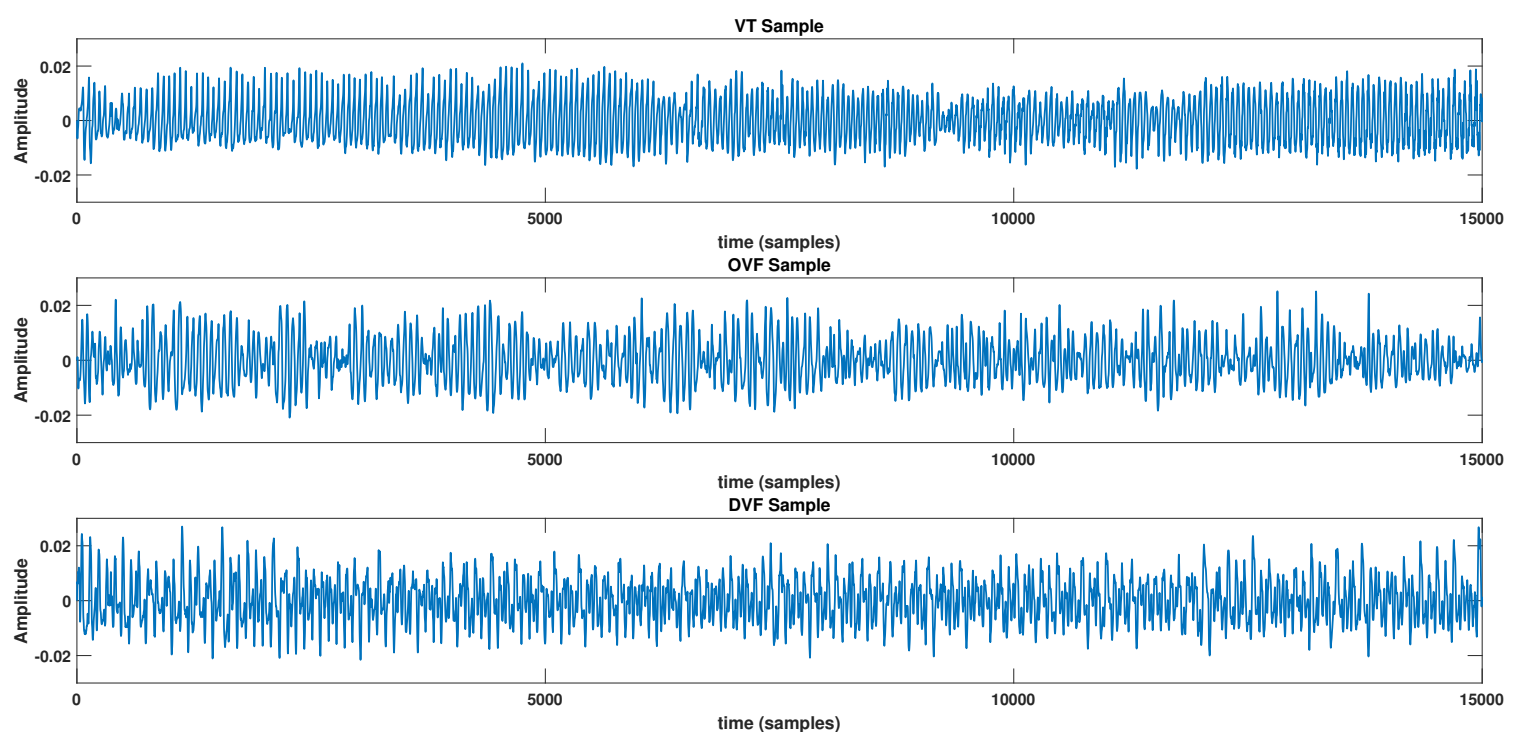

Figure 3.2: Sample ECG segments for VT, OVF and DVF from the proposed database.

\subsubsection{EMD \& Extracting IMFs}

Following the pre-processing stage, the ECG segments were processed by the EMD algorithm to decompose them into IMFs. The upper and lower envelope construction was done using the piecewise cubic Hermite interpolating polynomial (PCHIP) method [59]. The alternative was cubic spline method of interpolation. The PCHIP method was shown to have a reduced computational 
complexity compared to cubic spline method [59]. It also outperformed cubic spline in its ability to reduce overshooting in biological signals, and for these reasons it was selected for this analysis.

There were two stopping criteria used for the sifting process of the EMD algorithm. The first stopping criterion was a Cauchy type criterion called relative sifting tolerance (RelTol). Originally proposed by Huang et al. [40], the equation for RelTol was defined as [60]:

$$
\operatorname{RelTol}_{k}=\frac{\left\|c_{k-1}(t)-c_{k}(t)\right\|^{2}}{\left\|c_{k-1}(t)\right\|^{2}}
$$

Where $c_{k-1}(t)$ is the previous IMF, and $c_{k}(t)$ is the current $k^{t h} \mathrm{IMF}$. When the value of RelTol is less than the specified threshold, then the sifting process is ended. The criterion allows sifting to proceed until the point where the difference between successive IMFs is less than a specified limit. The threshold was set as 0.2 , which was the value recommended by Huang et al. [40]. This value was chosen such that the standard deviation between successive IMFs was large enough that the IMFs retain some physical sense in their amplitude and frequency modulations. The other stopping criterion was the maximum number of sifting iterations. This value represented the maximum number of sifting iterations an IMF may go through before the sifting process halts. The maximum number of iterations was set to 100. However, the signal characteristics of the ECG segments in the database dictated that this value was never reached in our analysis.

There were also a couple more EMD decomposition criteria that controlled the number of IMFs that were decomposed. The first criteria was the maximum number of extrema in the residual signal. If the number of maxima and minima in the residual is below the threshold, the residual is considered a monotonic function and the EMD algorithm is ended. This threshold was set to 1. This was based on the requirements of an IMF, in which a maxima and minima must exist within a signal to be considered an IMF. The second criterion was the maximum energy ratio (MER). The MER is the ratio between the energy of the original signal at the beginning of EMD and the mean envelope energy. The equation for this value was defined as [61]:

$$
M E R_{k}=10 \log _{10}\left(\frac{\|f(n)\|^{2}}{\left\|e_{k}(n)\right\|^{2}}\right)
$$


Where $f(n)$ is the original signal at the start of sifting, and $e_{k}(n)$ is the average envelope energy for the $k$ IMFs. This value provides a stopping mechanism that is independent of iteration count, and ensures that the energy of the IMFs do not become so small as to become insignificant. The threshold was set to $20 \mathrm{~dB}$, and if the MER exceeds it, the EMD algorithm will stop. This value was chosen such that the decomposition will halt when the energy of the residual signal becomes less than $1 \%$ of the original signal's energy.

For the long-term (in-hospital or offline) analysis, the extracted IMFs were organized from highest energy to lowest energy, and only the top 7 IMFs were chosen from every segment, and were carried forward. This was done because a different number of IMFs were extracted from each signal depending on its inherent complexity. The number of IMFs extracted from a given signal ranged from 7 to 9 . By limiting the analysis to the top 7 IMFs, it removes any bias created by comparing signals with varying numbers of IMFs. Because the vast majority of the ECG segments' energy ( $>90 \%$ ) was focused in those top 7 IMFs, minimal information was lost regarding signal characteristics. From these IMFs, features that quantify dynamic characteristics of VA were extracted to discriminate the 3 VA types.

For short-term (out-of-hospital) analysis, the IMFs were decomposed from each of the 4-s segments. The IMFs were sorted from highest to lowest energy, and only the top 3 IMFs were used in the analysis. Since the segments were shorter and thus less complex, the minimum number of IMFs extracted from a given segment was 3. Limiting the IMFs was done to ensure that a uniform number of IMFs were used from each segment. For the short-term analysis, the VA signals were first segmented into 4-s segments, and EMD was applied on each of them individually to simulate the real-time acquisition of data. Then features were extracted and a cumulative averaging strategy was employed to provide feedback on the type of arrhythmia with the evolution over time. From a practical implementation context, the method will perform cumulative averaging of features that capture VA dynamics and provide feedback with time progression. Although this approach is noninstantaneous, due to computationally efficient EMD, the approach lends well for near real-time 
applications. The next chapter will present the instantaneous features (via HS TFR) and their application for both long-term and short-term analysis.

\subsubsection{Feature Extraction}

Energy Based IMF Features

Based on the analysis of IMFs of VA ECG segments, it was observed a crucial clue lies in the way the energy of the signal is distributed over the IMFs for the different types of VA. This motivated us to look into features that can quantify the relative energy distribution over the IMFs. From the IMFs decomposed from each of the ECG signals, two energy ratio based features were computed. They were designed to quantify the distribution of energy across the set of IMFs. The first feature extracted was called the Energy Ratio Variance (ERV). This feature was inspired by previous work done by our research group [62], however using ICA. The ERV is calculated by finding the variance of the normalized energies for each of the IMFs used in the analysis. ERV was defined as:

$$
E R V=\frac{1}{k-1} \sum_{j=1}^{k}\left(E_{j}-\bar{E}\right)^{2}
$$

Where $E_{j}$ is ratio of the energy of the $j^{\text {th }}$ IMF normalized by the total energy of all IMFs used. The ERV feature quantifies the amount of complexity of a signal. For instance, a signal that has the majority of its energy concentrated around one IMF, i.e. mono-component, will in turn have a higher ERV than a signal that has its energy distributed more evenly over multiple IMFs, i.e. multi-component. The complexity of VT, OVF and DVF tends to be different, with VT having the lowest and DVF having the highest. Thus, we expect that the ERV feature will be able to quantify this difference between the groups effectively.

Another extracted feature similar to the ERV is the energy ratio skewness (ERS). Instead of taking the variance of the energy ratios of the IMFs, it computes the skewness measure of those ratios. We treat the energy ratio values as samples in a probability distribution. The ERS was 
calculated as [63]:

$$
E R S=\frac{1}{k} \sum_{j=1}^{k} \frac{\left(E_{j}-\bar{E}\right)^{3}}{\sigma^{3}}
$$

Where $\sigma^{3}$ is the cubed standard deviation of the normalized energy ratios. The ERS indicates how far away the energy ratios are spread from the mean value. A skew below the mean is negative, and a skew above the mean is positive. This feature also helps to quantify the amount of variability in the energy distribution of the IMFs for a given signal.

To use the ERV and ERS to track dynamic changes in VAs over time, they were calculated over sliding windows for each of the segments, windowing all the IMFs and computing one value for each window. Two different window sizes were used, which were 250 samples (1-s) and 1000 samples (4-s), with the slide width of 10 samples. Any window size may be chosen based on the time resolution desired, but 1-s was chosen because it was short enough to capture rapidly occurring changes over time, while 4-s was chosen to capture the slower changes over time. For illustration, Figure 3.3 shows the 1-s windowed ERV values for 3 different VA samples. It is apparent that the ERV values were the highest for VT, lower for OVF, and the lowest for DVF. While this tracking may not be instantaneous, it still indicates overall trends in energy distribution over time. Statistical measures may be extracted from the windowed ERV and ERS to help quantify the dynamic behaviour of the signals and compare differences between the arrhythmias.

\section{Statistical Measures}

From the windowed ERV and ERS, a number of statistical measures over time were calculated. Since it was desired to highlight differences in the characteristics of the arrhythmias, these statistical features will help quantify any observed differences. Features like mean, median, variance, RMS and slope of the time-windowed ERV and ERS features were computed. The equations for the features extracted from the windowed ERV are given below. The mean of the windowed ERV was calculated as: 

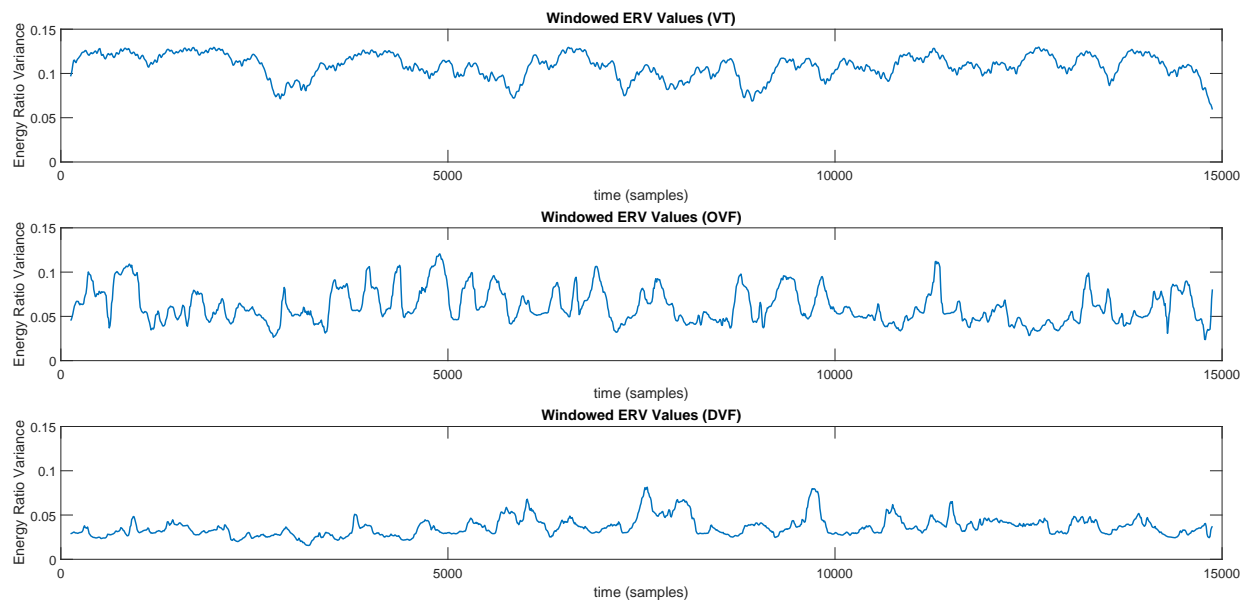

Figure 3.3: The sliding (1-s) windowed ERV values for a sample VT, OVF, and DVF signal.

$$
E R V_{\text {mean }}=\frac{1}{m} \sum_{j=1}^{m} E R V(j)
$$

The median of the windowed ERV was calculated as:

$$
E R V_{\text {median }}=E R V_{\text {sorted }}\left(\frac{m+1}{2}\right)
$$

Where $E R V_{\text {sorted }}(m)$ is the ERV values arranged from lowest to highest. The variance of the windowed ERV was computed as:

$$
E R V_{\text {variance }}=\frac{1}{m-1} \sum_{j=1}^{m}(E R V(j)-\overline{E R V})^{2}
$$

The RMS-value of the windowed ERV was computed as:

$$
E R V_{R M S}=\sqrt{\frac{1}{m} \sum_{j=1}^{m}\left[E R V(j)^{2}\right]}
$$

The slope of the windowed ERV was computed as:

$$
E R V_{\text {slope }}=\frac{E R V(\text { end })-E R V(1)}{t_{\text {end }}-t_{1}}
$$

Likewise, the statistical measures were also extracted from the windowed ERS. 
These values will indicate variations in the general trends of ERV and ERS over time. For instance, by computing variance of the windowed ERV, it allows us to quantify the variability in the energy complexity of these arrhythmias over time. For the long-term (in-hospital or offline) analysis, since we have access to long durations of VA segments in practice, we can perform quantification of dynamic characteristics of VA on a variety of ways including windowed analysis over shorter segments. Hence, in this study these features were extracted for both the 1-s and 4-s windowed ERV and ERS. For instance, the features denoted as 'ERV mean (4s)'describes the mean of the 4-s windowed ERV, whereas 'ERS median (1s)' defines the median for the 1-s windowed ERS. The ERV and ERS were also computed over the total length of each segment, and are denoted as 'ERV total' and 'ERS total'.

In the short-term analysis portion however, the ERV and ERS features were computed over windowed segments since in real-time scenarios access to prior data to perform analysis similar to long-term analysis is not available. The ERV and ERS were extracted over 4-s segments only. A window size of 4-s was chosen to make this analysis comparative to previous works that quantified VA organization over the same window size. Moreover sliding windows will not be efficient as the features in this chapter are non-instantaneous and hence is not expected to change drastically with sliding windows. However, to be true to providing immediate feedback for an 'out-of-hospital' scenario, we employed a cumulative averaging strategy to update the VA type as the time progresses. That is, this continuous update over cumulative temporal dynamics on arrhythmia detection can be controlled by the user. Also as there was no need to delineate between different window sizes, 'ERV variance', for example, in short-term analysis specifies the variance of the 4-s (segmented) windowed ERV. These statistical measures were computed similarly to long-term analysis, and then used to train the LDA-based pattern classifiers to discriminate between the 3 VA groups.

\subsubsection{Pattern Classification}

In order to discriminate the 3 VA types, a two stage binary classification scheme using an LDAbased classifier was implemented. In the first stage, the VT group was discriminated from the 
combined VF group (OVF and DVF). In the second stage, the misclassified VF samples were removed, and the remaining successfully classified VF samples were discriminated into OVF and DVF. The LOOCV method was also used to test the results of the classifier. The following section will be present the results for the long-term and short-term analysis.

\subsection{Results}

In this section the results of this analysis will be presented. It will be presented in two parts. The first part will contain the results of the long-term analysis for classifying the VAs over their full 1-minute duration for 'in-hospital' settings. The second part will present the short-term analysis results where the VA signals were segmented to provide a near real-time feedback on the VA classification for 'out-of-hospital' settings.

\subsubsection{Long-Term (In-Hospital or Offline) Analysis}

In the first classification stage, the VT group was classified from the VF group. In total, there were 22 VT signals and 39 VF signals. Using the LDA-based classifier, the best result was obtained using a combination of 3 features. The combination of features that produced better separation between VT and VF groups was the following: ERS total, ERV slope (4s), and ERV variance (1s). The boxplots for these 3 features are pictured below in Figures 3.4a, 3.4b, and 3.4c respectively.

In Figure 3.4a, it was observed that the ERS total feature was higher for the VT group compared to the VF group. The ERS gives an indication as to the amount of skew present in the energy ratios of the IMFs. The VT group generally had a more positive skew, because there is typically one dominant IMF that shifts the energy distribution above the mean. Whereas in the VF group, there is typically a more balanced distribution of energies across the IMFs, so the skew is not as pronounced. This result was consistent with VT being more mono-component and VF being more multi-component.

For the ERV slope (4s) feature, it was shown to be higher (more positive) for the VT group compared to the VF group in Figure 3.4b. The ERV slope gives insight into how the level of com- 

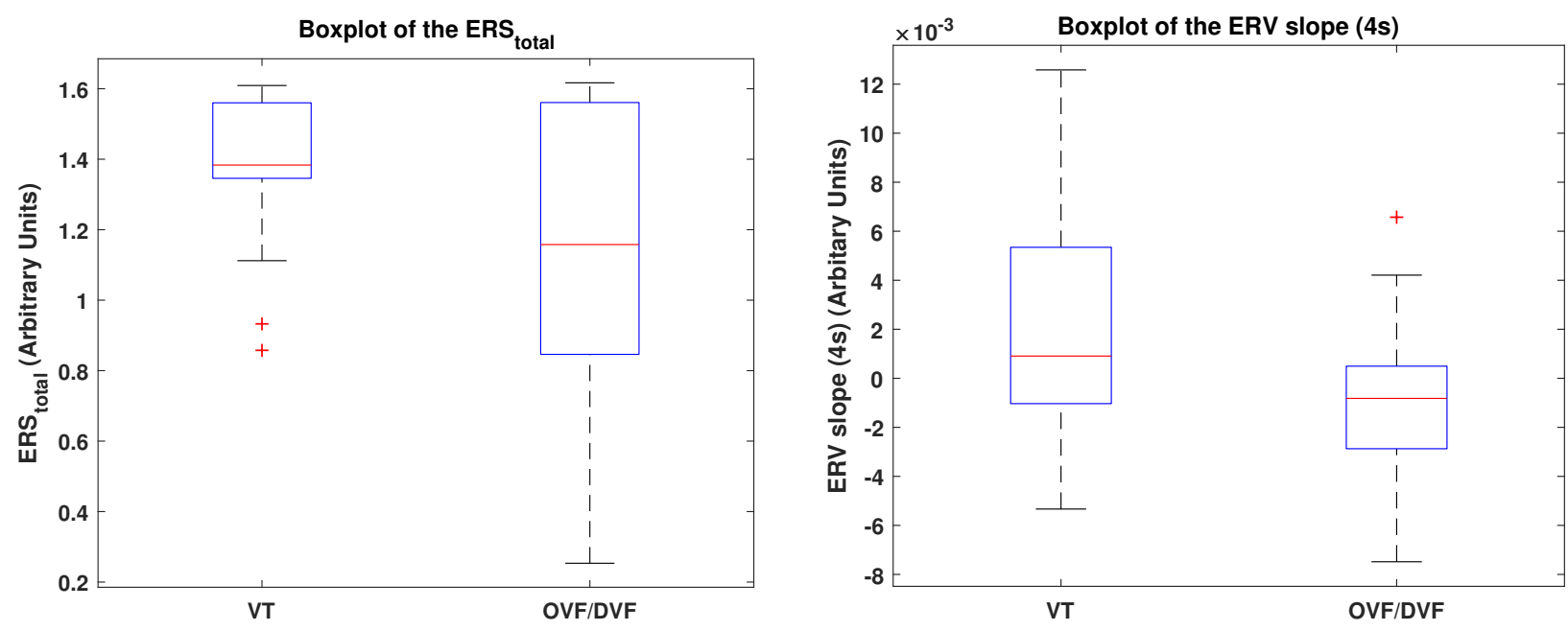

(a) ERS total.

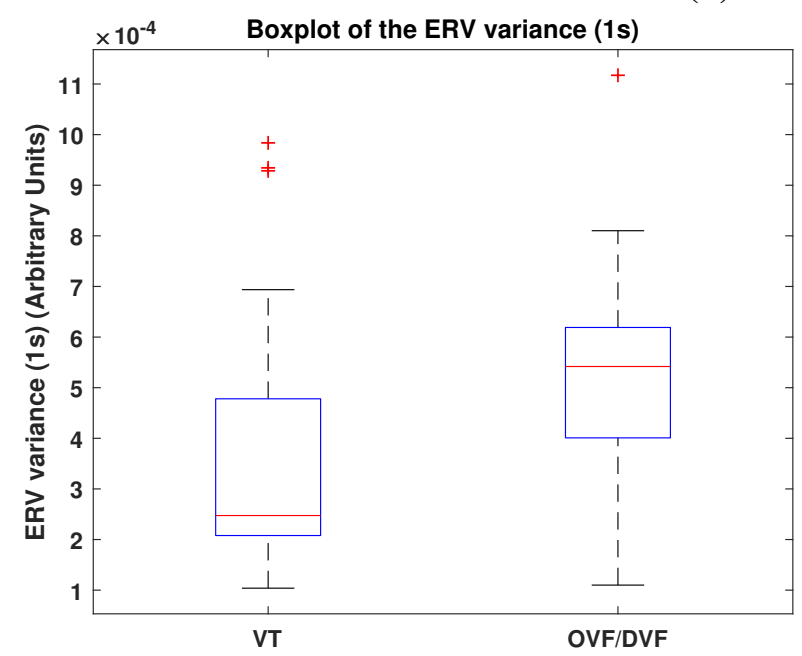

(c) ERV variance (1s).

Figure 3.4: The boxplots for the 3 features used for the VT vs. VF classifier. 
plexity within the VA segment is changing over time. In the VT group, the slope stays relatively close to zero and even strays positive, showing that the level of complexity is remaining almost unchanged or decreasing. However in the VF group, the slope more negative, indicating that the level of complexity is increasing. Over time, it is expected that VF will devolve and become more multi-component, resulting in a decreasing ERV value. Thus, this result aligned with previous expectations of VT and VF over time.

Thirdly, in the boxplot for ERV variance (1s) in Figure 3.4c, the VF group was shown to be higher than the VT group. ERV variance demonstrates the amount of volatility occurring in the organizational complexity of a signal over time. It is known that VF is a more disorganized phenomenon than VT, and thus it was expected that VF would have a higher ERV variance over time. The utilization of 1-s windows helped to capture any rapid changes occurring in the organization of the VAs over time. The ERV and ERS features used demonstrated that quantifying the distribution of energy among the IMFs proved useful for classifying VT and VF. This result validated the notion that the organizational complexity is a strong discriminating factor between these 2 arrhythmia types, showing that the VF was more organizationally complex than VT. Overall, this approach utilized the quantifiable difference in the energy concentrations across the IMFs to create a methodology with a strong capability of discriminating between VT and VF.

Table 3.1: VT vs. VF classification accuracy for leave-one-out cross-validated data.

\begin{tabular}{|c|c|c|c|c|}
\hline Method & Groups & VT & VF & Total \\
\hline Cross-validated & VT & $\mathbf{1 9}$ & 3 & 22 \\
\hline & VF & 4 & $\mathbf{3 5}$ & 39 \\
\hline$\%$ & VT & $\mathbf{8 6 . 4}$ & 13.6 & 100 \\
\hline & VF & 10.3 & $\mathbf{8 9 . 7}$ & 100 \\
\hline
\end{tabular}

A summary of the cross-validated results using the above 3 features is shown in Table 3.1. The classification accuracy achieved was $88.5 \%$. The standard measures of sensitivity and specificity are not shown, since those measures imply that there is a normal and an abnormal case. However, in this analysis both cases are considered abnormal, so those measures would not be meaningful here. As seen in Table 3.1, 3 out of 22 VT samples and 4 out of 39 VF samples were misclassified. 
This result was as expected, since it was well-documented in literature that VT may be classified from VF with high accuracy using simple features. However, a challenging problem still remains in classifying PVT and OVF. These two VAs are quite similar in structural and temporal complexity, and there were PVT samples present in the database. So, achieving a high classification accuracy for this stage was a promising result.

The proposed approach also has additional advantages compared to previous methods:

1. The first advantage lies in the completely data-driven nature of this approach. Since EMD was used to derive IMF energy-based features, the results did not rely on any pre-defined basis function to perform the analysis. The IMFs were decomposed strictly on the basis of their inherent properties and the natural oscillatory modes present within the signals themselves.

2. Another benefit of this work is that it has a very low computational complexity relative to previous methods. EMD may be implemented with a computational complexity on the same order as the FFT [16]. This means that due to its speed this method could be implemented in near real-time applications to aid in discrimination of VAs so that EMS or ICDs provide the correct treatment modalities for patients.

3. The third advantage is the ERV/ERS features were calculated for short 1-s and 4-s intervals over a sliding window, and it helped in the discrimination between VT and VF. This indicated that this may be useful for short-term analysis as well. By computing these measures over short windows, this approach has the potential to provide low-latency feedback to EMS personnel on the arrhythmia of patients to help them decide on the most appropriate treatment options to use. This will be demonstrated further in the subsequent section on short-term analysis.

The ROC curve for the total classifier is shown in Figure 3.5. The ROC curve's global pattern indicated that the classifier had strong discriminating power for these two groups of data. The AUC was 0.911 , which indicated a strong classifier performance. The p-value for the linear discriminant 


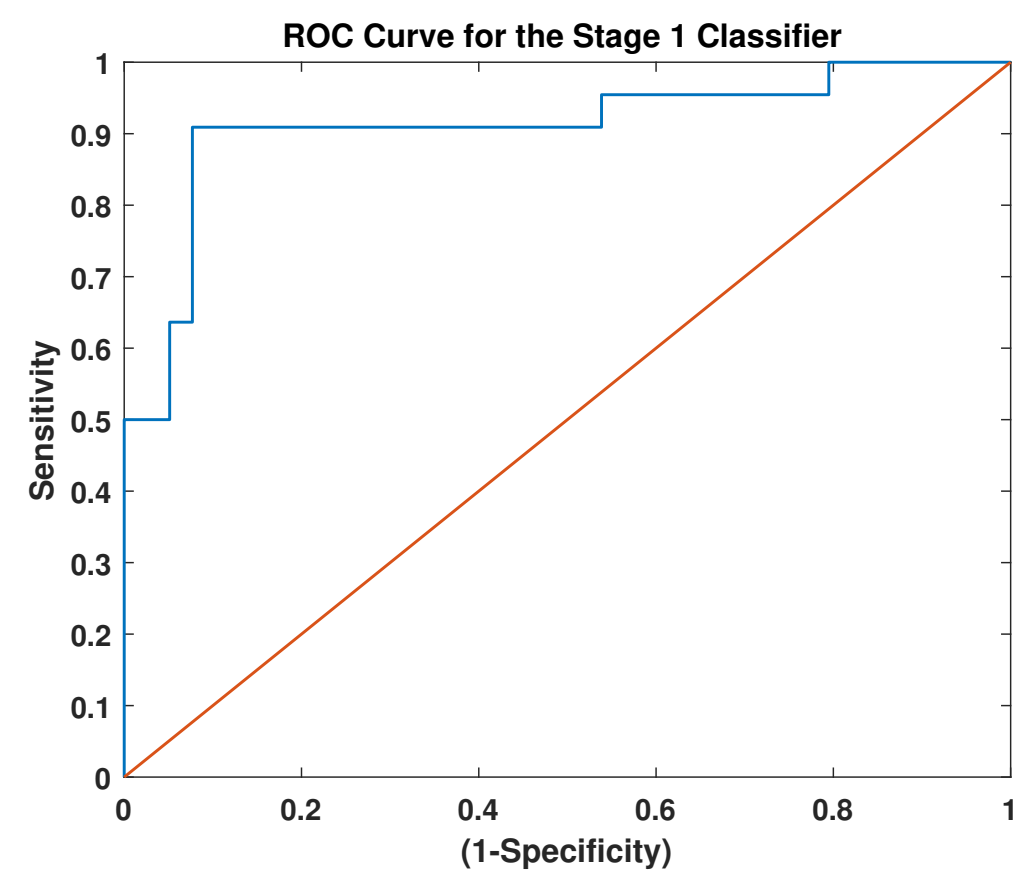

Figure 3.5: The ROC curve for the VT vs. VF classifier.

scores of this classifier was also significant $(p<0.01)$.

In the second classification stage, the OVF group was classified from the DVF group. Discriminating OVF and DVF has proven to be a difficult problem, due to the natural overlap between them in nature. OVF will often devolve into DVF over time, making it challenging to identify exactly when the distinction has occurred. Due to these challenges, sophisticated measures are often necessary to classify these VF sub-types.

The four misclassified VF samples from the previous stage was removed prior to this stage. In total, there were $15 \mathrm{OVF}$ and $20 \mathrm{DVF}$ signals used. The single feature that produced the highest discrimination between OVF and DVF was: ERS median (1s). The boxplot for this feature is shown below in Figure 3.6.

In the boxplot in Figure 3.6, the median of the windowed (1s) ERS was higher for the OVF group compared to the DVF group. The ERS median feature gave insight into the average amount of skew present in the IMFs' energy ratios over the sliding segments of the signal. The OVF group 


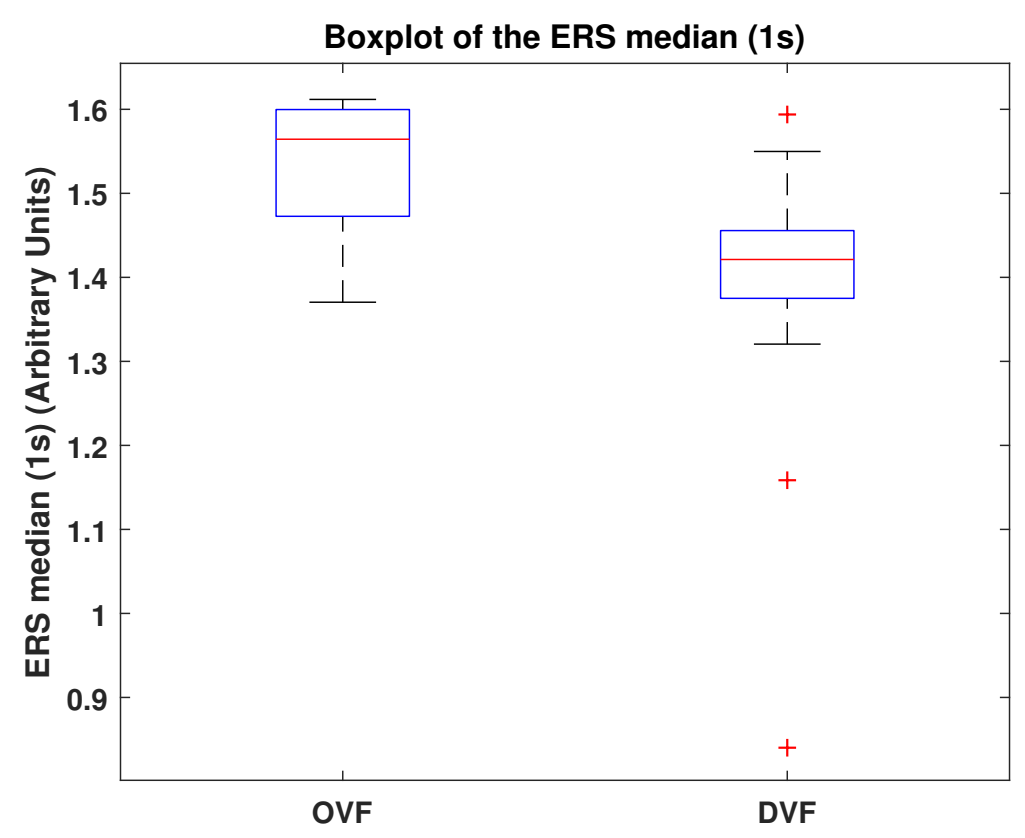

Figure 3.6: The boxplot for the ERS median (1s) feature between OVF and DVF groups.

presented with a more positive skew than the DVF group, indicating a greater amount of shift in the energy ratios above the mean. Since it was expected for DVF to be more organizationally complex and multi-component than OVF, this result made sense. A higher level of organizational complexity was observed for the DVF group compared to the OVF group.

The table summarizing the cross-validated results is shown in Table 3.2. The overall classification accuracy obtained here was $80.0 \%$. In Table 3.2, it is shown that 3 out of 15 OVF and 4 out of 20 DVF signals were misclassified. Given the difficulty of this classification task, a drop in performance compared to the first classification stage was expected. However, classifying OVF and DVF is a challenging problem with many potential implications, and so it is of more significance in VA treatment.

Table 3.2: OVF vs. DVF classification accuracy for leave-one-out cross-validated data.

\begin{tabular}{|c|c|c|c|c|}
\hline Method & Groups & OVF & DVF & Total \\
\hline Cross-validated & OVF & $\mathbf{1 2}$ & 3 & 15 \\
\hline & DVF & 4 & $\mathbf{1 6}$ & 20 \\
\hline$\%$ & OVF & $\mathbf{8 0}$ & 20 & 100 \\
\hline & DVF & 20 & $\mathbf{8 0}$ & 100 \\
\hline
\end{tabular}




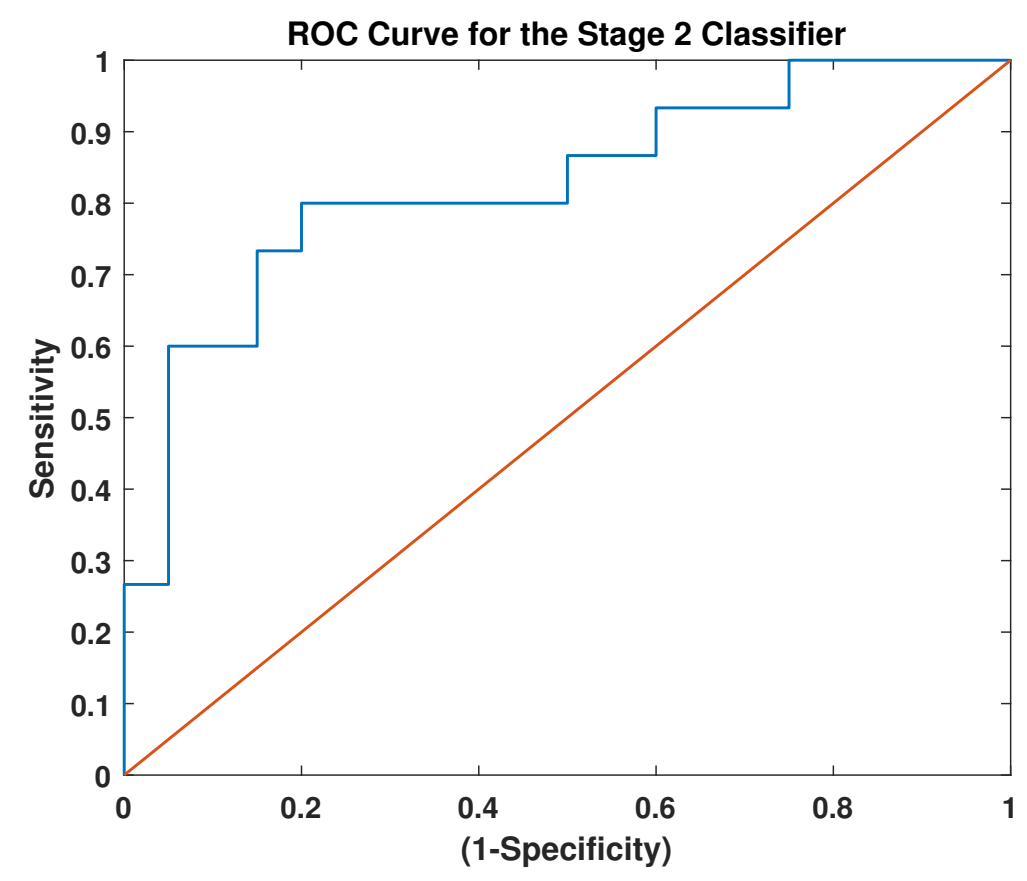

Figure 3.7: The ROC curve for the OVF vs. DVF classifier.

The ROC curve for the classifier used in this secondary stage is shown in Figure 3.7. The global pattern remained well above the diagonal bisector, indicating a robust discriminating power. The AUC for this classifier was 0.827 , which demonstrates a strong classifier performance. The p-value for the single feature used to train the classifier was also statistically significant $(p<0.01)$. These statistical measures helped to verify the successful classification performance. In addition, this approach achieved a much faster computational speed than previous methods, suiting it to many real-world applications like programming into ICDs.

In order to verify the efficacy of these results, a comparative analysis was performed where previous well-known features from literature were used to compare the results obtained. The comparative features extracted were: dominant frequency (DF), temporal entropy (EN), and spectral entropy (SE). The features were extracted over 1-s and 4-s sliding windows similar to how the ERV and ERS features were extracted. They were used to train the same LDA-based classifier, and the classification results were obtained by LOOCV. For the first stage of VT vs. VF classification, four features were used to train the classifier: DF mean (1s), EN median (4s), SE mean (4s), and SE 
median (1s). The summary of the classification results is shown below in Table 3.3. In the second classification stage between OVF and DVF, the best performing feature used to train the classifier was: DF median (1s). The summary table of the results is shown in Table 3.4.

Table 3.3: VT vs. VF classification accuracy for the comparative features.

\begin{tabular}{|c|c|c|c|c|}
\hline Method & Groups & VT & VF & Total \\
\hline Cross-validated & VT & $\mathbf{2 0}$ & 2 & 22 \\
\hline & VF & 4 & $\mathbf{3 5}$ & 39 \\
\hline$\%$ & VT & $\mathbf{9 0 . 9}$ & 9.1 & 100 \\
\hline & VF & 10.3 & $\mathbf{8 9 . 7}$ & 100 \\
\hline
\end{tabular}

For the first stage, a total classification accuracy of $90.2 \%$ was achieved, and in the second stage a total accuracy of $66 \%$ was obtained. Compared to the results achieved in the proposed methodology, the comparative features achieved a slightly better classification result for VT versus VF, improving by 1 VT sample. However, the comparative features required four features to train the stage 1 classifier, whereas only 3 features were needed using the proposed method. Also, the SE feature was computationally expensive to extract, whereas the proposed features were not. In addition it should be noted that it is a well known fact that rhythm or frequency features are highly discriminatory for VT and VF classification which might explain spectral features performing better than the proposed non-spectral energy features. In the second stage between OVF and DVF, the proposed approach significantly outperformed the comparative features with an accuracy of $80 \%$ compared to $66 \%$. As well, none of the comparative features extracted for OVF vs. DVF were statistically significant, whereas the ERS median feature was.

Table 3.4: OVF vs. DVF classification accuracy for the comparative features.

\begin{tabular}{|c|c|c|c|c|}
\hline Method & Groups & OVF & DVF & Total \\
\hline Cross-validated & OVF & $\mathbf{1 1}$ & 6 & 17 \\
\hline & DVF & 6 & $\mathbf{1 2}$ & 18 \\
\hline$\%$ & OVF & $\mathbf{6 4 . 7}$ & 35.3 & 100 \\
\hline & DVF & 33.3 & $\mathbf{6 6 . 7}$ & 100 \\
\hline
\end{tabular}




\subsubsection{Short-Term (Out-of-Hospital) Analysis}

In the second part of this proposed approach, short-term analysis was performed by analyzing the 60-s ECG segments over shorter 4-s windows. By analyzing 4-s segments cumulatively incremented over time, it would allow for the user to pause and resume the analysis at any time and obtain a diagnosis regarding the condition of a patient's VA. This would provide EMS personnel with near real-time feedback on the patient's progression and response to any treatments administered. In order to test the robustness of this methodology, the results were obtained using 3 different time durations for the ECG segments: the first 20-s, the first 40-s and the total 60-s of the VA segments. Doing so would allow for simulation and comparison of the performance over multiple time points during real world scenarios.

In order to track the progression of the VAs over time using ERV and ERS, they were calculated using a cumulative averaging method. Cumulative averaging takes the preceding value and averages it with the current value to get the next value. By doing this, it is possible to observe average changes occurring in the ERV and ERS over the duration of the VA signals. Using the 4-s segments of each 60-s VA signal, the initial ERV and ERS values were computed for the first 4 seconds, and then all subsequent values were calculated using cumulative averaging. The formula to calculate the cumulative average value ERV and ERS are defined below:

$$
\begin{aligned}
& E R V(m)=\frac{E R V(m)+E R V(m-1)}{2} \\
& E R S(m)=\frac{E R S(m)+E R S(m-1)}{2}
\end{aligned}
$$

Where $E R V(m)$ is the current ERV value, and $E R V(m-1)$ is the preceding ERV value. Simulating a real world scenario, cumulative averaging will provide the EMS personnel with an update on the VA type/progression at any given time (however restricted to $4 \mathrm{~s}$ window updates). The features of mean, median, variance, RMS, and slope were calculated identically as in Equations 3.5 to 3.9 used for long-term analysis. An additional feature used in this section was the ' $E R V_{\text {final }}$ ' or 
' $E R S_{\text {final }}$ ', which were the final cumulative average values obtained over the choice of monitoring duration. Using these features, the LDA-based classifier was trained, and the VA segments were classified in a two-stage binary scheme, initially classified into VT and VF, and then the correctly classified VF segments into OVF and DVF.

The results of the 3 duration trials is shown in Table 3.5. For the 20-s duration trial, the stage 1 classifier achieved the best performance when trained with the a single feature: ERS median. The total classification accuracy obtained was $63 \%$. In the second classification stage, the misclassified VF samples were removed, and the best result was achieved using the ERV median feature. The total cross-validated accuracy achieved there was $72 \%$. For the 40 -s duration trial, the stage 1 classifier was trained using 2 features: $E R V_{\text {final }}$ and ERV variance. The classification accuracy obtained was 79\%. In the second classification stage, the 9 misclassified VF samples were removed, and the classifier was also trained with 2 features: ERS mean and ERS variance. The total accuracy achieved in that stage was 67\%. Lastly in the 60-s duration trial, the initial stage classifier was trained with 3 features to obtain the best result: ERS mean, ERV variance, and $E R V_{\text {final }}$. The total accuracy achieved in stage 1 was $72 \%$. In the secondary classification stage, the 10 misclassified VF segments were taken out, and the classifier was trained with 4 total features: ERV mean, ERV median, ERS median, and ERS RMS. In total, the classification accuracy achieved in this stage was $81 \%$.

In order to check the validity of these results, the same comparative features from the long-term analysis were also used here to see their performance. The summary of the classification results can be seen in Table 3.6. For the 20-s duration trial, the first stage classifier was trained using 2 features to obtain the best result: DF mean and $E N_{\text {final }}$. The total classification accuracy obtained was $83 \%$. In the second classification stage, with the misclassified VF samples removed, the classifier was trained using one feature: $S E_{\text {final }}$. The total cross-validated accuracy obtained there was $64 \%$. For the 40-s duration trial, the initial stage classifier was trained using 2 features: DF mean and EN mean. The total classification accuracy for the first stage was $88 \%$. In the second classification stage, the classifier was trained using one feature: $D F_{\text {final }}$. The total classification 
Table 3.5: Classification table for 20-s, 40-s, and 60-s duration results using leave-one-out crossvalidated data.

\begin{tabular}{|c|c|c|c|c|c|c|c|c|}
\hline Duration & Groups & $\mathrm{VT}$ & $\overline{V F}$ & Total & Groups & OVF & $\overline{\text { DVF }}$ & Total \\
\hline \multirow[t]{2}{*}{$20-S$} & $\mathrm{VT}$ & 16 & 6 & 22 & OVF & $\overline{6}$ & 2 & 8 \\
\hline & $\mathrm{VF}$ & 18 & 21 & 39 & $\overline{D V F}$ & 4 & 9 & 13 \\
\hline \multirow[t]{2}{*}{$\%$} & VT & 72.7 & 27.3 & 100 & OVF & 75.0 & 25.0 & 100 \\
\hline & $\mathrm{VF}$ & 46.2 & 53.8 & 100 & DVF & 30.8 & 69.2 & 100 \\
\hline \multirow[t]{2}{*}{$40-s$} & $\mathrm{VT}$ & 18 & 4 & 22 & OVF & $\overline{6}$ & 3 & 9 \\
\hline & $\mathrm{VF}$ & 9 & 30 & 39 & $\overline{\mathrm{DVF}}$ & 7 & 14 & 21 \\
\hline \multirow[t]{2}{*}{$\%$} & VT & 86.4 & 13.6 & 100 & OVF & 66.7 & 33.3 & 100 \\
\hline & $\mathrm{VF}$ & 23.1 & 76.9 & 100 & DVF & 33.3 & 66.7 & 100 \\
\hline \multirow[t]{2}{*}{ 60-s } & $\mathrm{VT}$ & 15 & 7 & 22 & OVF & 8 & 3 & 11 \\
\hline & VF & 10 & 29 & 39 & DVF & 2 & 16 & 18 \\
\hline \multirow[t]{2}{*}{$\%$} & $\mathrm{VT}$ & 68.2 & 31.8 & 100 & OVF & 72.7 & 27.3 & 100 \\
\hline & $\overline{\mathrm{VF}}$ & 25.6 & 74.4 & 100 & DVF & 11.1 & 88.9 & 100 \\
\hline
\end{tabular}

accuracy achieved was $60 \%$. Finally in the 60-s duration trial, the first stage classifier was trained using 3 total features: DF median, EN mean and SE median. The total cross-validated accuracy in this initial stage was $90 \%$. In the second classification stage, with the 6 misclassified VF samples removed, the classifier was also trained with 3 features for the best result: EN median, $D F_{\text {final }}$, and DF median. In that stage, the total classification accuracy achieved was $76 \%$.

When comparing the results of the proposed features for the 3 trials, it was evident that the best results were obtained over the longest duration trial of 60-s. In general, the classification accuracies for both stage 1 and stage 2 improved the longer the time duration was. This is because the ERV and ERS features were better able to capture the dynamic changes of the VAs over time the longer the segments continued. However, it was noted that the OVF vs. DVF accuracy for the 20-s duration trial exceeded the results of the 40-s trial, and the accuracy for VT vs. VF was higher for the 40-s trial than the 60-s trial. This may be explained by the fact that the VA segments were not uniform throughout its total length, and evolution in the VA type may be occurring over time. Depending on where in time the analysis began, the results of the classification could change. Because the VA segments are considered 'mostly' OVF or DVF over their full 60-s length, it was 
Table 3.6: Comparative results classification table for $20-\mathrm{s}, 40-\mathrm{s}$, and $60-\mathrm{s}$ duration results using leave-one-out cross-validated data.

\begin{tabular}{|c|c|c|c|c|c|c|c|c|}
\hline Duration & Groups & VT & VF & Total & Groups & OVF & DVF & Total \\
\hline $\mathbf{2 0 - s}$ & VT & $\mathbf{1 8}$ & 4 & 22 & OVF & $\mathbf{1 1}$ & 6 & 17 \\
\hline & VF & 6 & $\mathbf{3 3}$ & 39 & DVF & 6 & $\mathbf{1 0}$ & 16 \\
\hline$\%$ & VT & $\mathbf{8 1 . 8}$ & 18.2 & 100 & OVF & $\mathbf{6 4 . 7}$ & 35.3 & 100 \\
\hline & VF & 15.4 & $\mathbf{8 4 . 6}$ & 100 & DVF & 37.5 & $\mathbf{6 2 . 5}$ & 100 \\
\hline & \multicolumn{7}{|l|}{} \\
\hline $\mathbf{4 0 - s}$ & VT & $\mathbf{1 9}$ & 3 & 22 & OVF & $\mathbf{1 1}$ & 6 & 17 \\
\hline & VF & 4 & $\mathbf{3 5}$ & 39 & DVF & 8 & $\mathbf{1 0}$ & 18 \\
\hline$\%$ & VT & $\mathbf{8 6 . 4}$ & 13.6 & 100 & OVF & $\mathbf{6 4 . 7}$ & 35.3 & 100 \\
\hline & VF & 10.3 & $\mathbf{8 9 . 7}$ & 100 & DVF & 44.4 & $\mathbf{5 5 . 6}$ & 100 \\
\hline & \multicolumn{7}{|l|}{} \\
\hline $\mathbf{6 0 - s}$ & VT & $\mathbf{2 1}$ & 1 & 22 & OVF & $\mathbf{1 2}$ & 4 & 16 \\
\hline & VF & 6 & $\mathbf{3 3}$ & 39 & DVF & 4 & $\mathbf{1 3}$ & 17 \\
\hline$\%$ & VT & $\mathbf{9 5 . 5}$ & 4.5 & 100 & OVF & $\mathbf{7 5 . 0}$ & 25.0 & 100 \\
\hline & VF & 15.4 & $\mathbf{8 4 . 6}$ & 100 & DVF & 23.5 & $\mathbf{7 6 . 5}$ & 100 \\
\hline
\end{tabular}

expected that the classification results would be the most stable for the 60-s trial. The proposed approach at times performed better at classifying OVF and DVF than VT and VF in the same trial. Although the result for VT versus VF were not ideal, it was promising to see that the proposed method worked comparatively well at discriminating OVF and DVF, the more pressing problem.

When contrasting the results between the proposed ERV/ERS features and the comparative features, the comparative features outperformed the proposed features in VT vs. VF classification for all three trials. Again, as explained earlier this is due to the fact that rhythm or frequency features are highly discriminatory for VT and VF classification which is evident that the spectral features performed better than the proposed non-spectral energy features. However, the proposed features outperformed the comparative features in the OVF vs. DVF classification during all three trials. This trend indicated that the comparative features were better able to solve the simpler problem of separating VT and VF by spectral differences. However, the proposed features were better suited for the more challenging problem of classifying OVF and DVF. Although, it should be noted that since the VT/VF classification accuracy was lower for the proposed features, there were less VF 
samples to classify in stage 2. Nevertheless, this result showed that the ERV and ERS features were able to capture dynamic changes and differences in the organizational complexity of the two VF sub-types.

Although the results of the short-term analysis were not as strong as the long-term analysis, this approach has some key advantages. Firstly, this short-term approach is able to quantify the VAs over time, and provide almost instant feedback to the user when desired. The short-term analysis may be halted and resumed at any time, allowing the user to receive the diagnostic feedback without having to wait for the full 60-s duration as in long-term analysis. Although the classification results tend to improve the longer the analysis window is, it still provides more flexibility for feedback applications. Secondly, because this EMD-based approach is computationally efficient, it would be able to provide near real-time feedback to EMS personnel in emergency situations. This methodology could be used by EMS to monitor the condition of a patient during the course of treatment, and provide diagnostic feedback to aid them in selecting and altering treatment options depending on the results. For instance, this approach could be used to check the condition of a patient prior to and after a defibrillation to see how the patient's heart responded to the shock therapy. If the results were positive, the personnel could change the treatment to pacing, or if it was shown to be unsuccessful they could administer another shock. Overall, this proposed methodology showed potential to be useful for a number of clinical applications.

\subsection{Discussion}

During the long-term analysis, a high classification accuracy of $88.5 \%$ was achieved for VT vs. VF classification. This was in alignment with previous works that attempted to solve the same problem $[8,15]$. Unlike previous approaches that utilized decomposition techniques predicated on the choice of a pre-defined basis function, the EMD algorithm decomposes the data into IMFs based on the inherent properties of the data itself. The IMFs generated are the natural oscillatory modes present within the signal. The other benefit of utilizing EMD is its low computational complexity. Because of this, it was able to decompose the 1-minute ECG signals into IMFs swiftly, and facili- 
tate the extraction of dynamic features. The windowed ERV features extracted over 1-second and 4-second intervals on a sliding window (10 samples) also aided strongly in the classification task. This demonstrated the potential of these energy-ratio based features to quantify the complexity of VT and VF over short time-spans. This proposed approach could be used to provide feedback to assist medical personnel in diagnosing VAs and selecting the most appropriate therapy option for patients, or by doctors for post-analysis in offline settings.

In the second classification stage between OVF and DVF, a classification accuracy of $80 \%$ was achieved. This result was comparable to previous studies that attempted this challenging classification problem. Due to the natural overlap between OVF and DVF, discriminating these two groups required a more sophisticated approach. In a previous work by Balasundaram et al., they used wavelet-based features and SVD to classify OVF from DVF [8]. The features used by Balasundaram et al. [8] were also tested on the database used in this work. In the initial stage of VT versus VF, a total classification accuracy of $93 \%$ was obtained, and in the second stage of OVF versus DVF, a total accuracy of $84 \%$ was achieved. These results showed an improvement over the results achieved by this proposed approach. However, this proposed approach has a few key advantages. The computational time required for Balasundaram's approach was much greater. When comparing our approaches on one 60-s signal with the same hardware and software environment, this proposed approach was approximately 500 times faster. This makes the proposed method more suitable for providing near real-time feedback. Also, this approach is completely data-driven, since EMD decomposed the ECG segments into IMFs based solely on the inherent properties of the segments themselves. Balasundaram's wavelet-based features on the other hand required the choice of a wavelet basis function, which also makes their approach less adaptable to application on different VA databases.

Bai et al. also performed classification of NSR, VT, and VF using and EMD based approach [15]. They were also able to achieve a high classification accuracy for discriminating those 3 rhythms. However, the problem of classifying OVF and DVF is much more challenging, and 
possesses implications that are more significant for impacting resuscitation success. This proposed approach was able to discriminate OVF and DVF successfully using a computationally efficient approach.

Especially within cases of VF, selection of the most suitable treatment options is crucial to the survival of patients. This approach provides a diagnostic tool capable of assisting EMS personnel in classifying sub-classes of VF in near real-time, aiding the selection of appropriate treatment options. This approach could also be implemented in ICDs, automatically diagnosing the type of arrhythmia a patient is experiencing and administering the correct therapy modality (electrical pacing or shock).

The short-term analysis approach, by its windowed analysis of the VA segments, was capable of performing near real-time classification of VAs, including VF. To the author's knowledge, this is the first proposed approach that attempted to used EMD-based features to sub-classify VF for near real-time applications. To test the results, a series of three trials was performed, one for 20-s, 40-s and 60-s durations. It was shown that as the length of duration increased, the classification results seemed to improve. This result suggested that the longer the duration, the better able the ERV and ERS features were at capturing the dynamic behaviour of the VAs over time. The features extracted captured the global pattern within each 4-s segment, and the cumulative averaging method introduced a relative difference measure that captured the progression of these values over time. The proposed features performed well in the 60-s duration trial, which aligned with the positive results obtained in the long-term analysis. Although the results were not as strong over the 40-s and 20-s trials, this approach showed the potential for some useful applications. Using these near real-time features to perform cumulative averaging meant that the analysis could be halted at any time and a diagnosis may be obtained. It may also be resumed at any time and perform further analysis. The only caveat being that it needed a 4-second buffer in order to calculate the initial ERV and ERS values. 
This approach could be used to monitor the VA of a patient in 'out-of-hospital' settings, passively acquiring data until the EMS decide that they want to check the diagnosis. This would give the EMS a real-time diagnostic feedback on the condition of a patient. They could acquire a diagnostic reading before administering a treatment, and then re-diagnose the patient to see if the treatment had any effect or not. This method could also be used by ICDs to quickly diagnose the VA of patients and aid in the proper selection of either pacing or shock therapies to return a patient heart rhythm to normal. Overall, the real-time application of this approach makes it stand out among many other VA classification techniques, and with further testing and validation could hopefully be used in the field to help improve the survival rates of VA patients during emergency scenarios.

The proposed approach provided the promising beginnings of a potential data-driven approach for classifying VAs. However, in order for this approach to be truly data-driven, a much larger VA database containing hundreds of segments would be required to train the system thoroughly. However, due to the complex ethical and practical constraints of acquiring human VF data, obtaining such a database was not possible. Due to the lethal nature of VF, acquiring data from live patients is very challenging. In spite of these challenges, a database containing 3960 -s long VF segments was obtained. This is quite expansive when compared to previous works.

\subsection{Chapter Summary}

In this chapter, we presented the results of long-term and short-term analysis of VAs using noninstantaneous features. A two-stage binary classification scheme was used, where initially VT was classified from VF, and secondly the correctly classified VF signals were sub-classified into OVF and DVF. Energy-ratio based features called ERV and ERS were extracted from the IMFs (decomposed by EMD) of the ECG segments, and were used to train the LDA-based classifier. For long-term analysis, an accuracy of $88.5 \%$ was achieved in the first stage, and in the second stage an accuracy of $80 \%$ was obtained. The significance of this approach stems from the fact that it is a completely data driven decomposition, based on the intrinsic properties of the data itself, and that it 
is far less computationally expensive than other previous methods used for this application. In the short-term analysis, near real-time features were used to detect VAs over 3 trials of different time durations. This method showed promise in providing near real-time feedback to medical personnel on the progression of VAs, and assist them in selecting and modifying treatment options. That gives this proposed approach the potential for use by EMS to aid in diagnosing arrhythmias and choosing appropriate treatment options. 


\section{Chapter 4}

\section{EMD Instantaneous Features for VA Classification and Trends Analysis}

$\mathrm{I}$

$\mathrm{N}$ the previous chapter, although for the short-term analysis we classified VAs using windowed segments and cumulative averages of features, these features do not qualify as true instantaneous markers of VA progression. This chapter will present EMD based instantaneous features for VA characterization. These instantaneous features were extracted from the HS, which was constructed from the decomposed IMFs. These instantaneous features allow for the quantification of the evolution of the organizational changes occurring in the signals, which may aid in the discrimination of these arrhythmias, particularly in sub-categorization of VF. Throughout this chapter, we will present the results of using instantaneous TF features to classify VAs. In the first part of the chapter, the results of using instantaneous features for long-term analysis aimed to assist clinicians 'in-hospital' will be presented. This approach will analyze the VA segments over their full duration to characterize their dynamic behavior over time. We will attempt to identify markers that will aid doctors during 'in-hospital' settings to more accurately diagnose VA types/progressions and plan their choice and modification of treatment options. The next part of the chapter will focus on short-term analysis directed for use in 'out-of-hospital' emergency settings. A trends analysis will be performed, where the instantaneous features will be computed over sliding segments of the ECG and used cumulatively in a scoring method to track the trends of VAs over time. These identified trends will help to inform EMS personnel about the progression of patients' conditions and provide feedback for the selection of appropriate therapy options in the field. Lastly, the long-term 


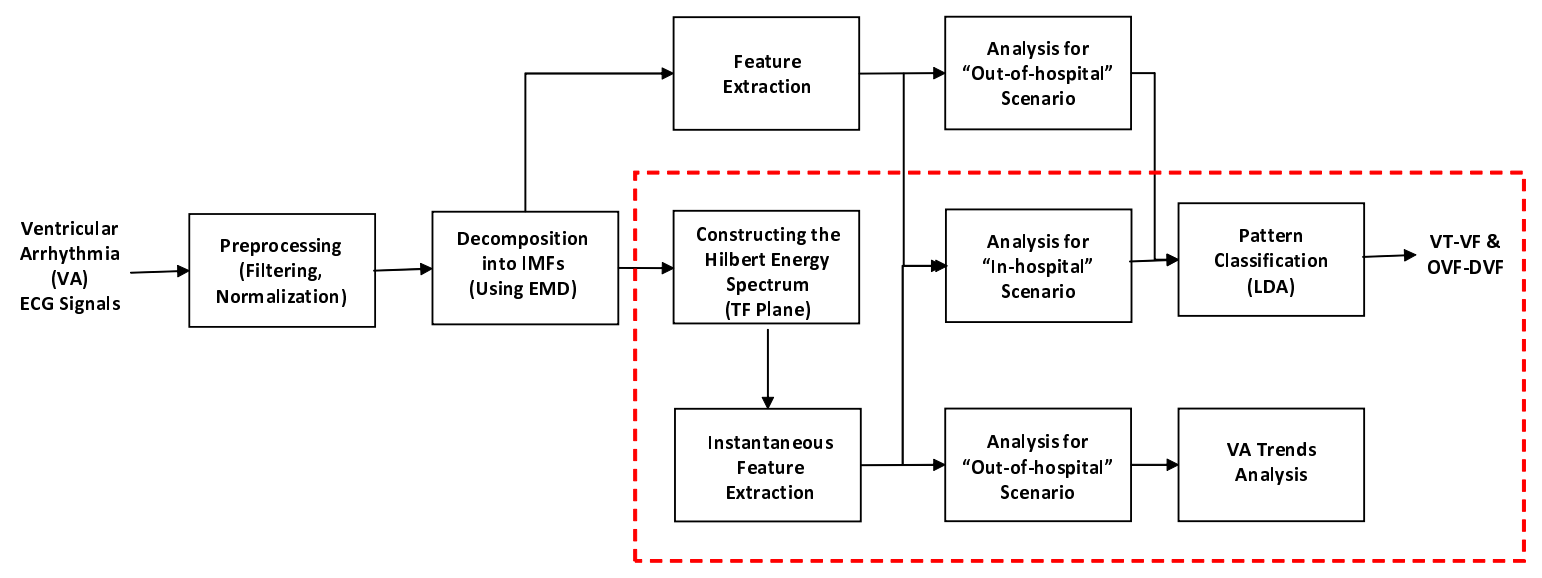

Figure 4.1: Block diagram highlighting the proposed work discussed in Chapter 4.

analysis parts used in Chapter 3 and Chapter 4 will be combined to produce a classification scheme for VT-VF and OVF-DVF. The block diagram highlighting this part of our study is shown below in Figure 4.1.

\subsection{Methodology}

This section will present the entire procedure for the approach presented in this chapter. It will start with a review of the the ECG database that was used. Then the construction of the HS and its parameters will be explained. Next, the feature extraction methods will be discussed along with the equations and methods used to acquire the features. This section will conclude with an explanation of the pattern classification techniques to be used to test the results of this proposed approach.

\subsubsection{ECG Database \& Pre-Processing}

The same database that was used in Chapter 3 was also utilized here. The 61 VA segments consisting of 22 'mostly' VT, 17 'mostly' OVF, and 22 'mostly' DVF segments of 60-s length were used. The long duration of these VA segments means that natural variations will occur that may be captured over time by the extracted instantaneous TF features. By capturing the dynamic changes in the temporal and spectral behaviour, this will help to discriminate between the 3 types of VAs. 
The ECG segments were band-pass filtered between 0.3 and $30 \mathrm{~Hz}$ to remove low frequency drift and high-frequency oscillations as previously.

These 60-s VA segments were also windowed for use in the short-term VA trend analysis. This is because we also wished to devise a near real-time feedback method for real world scenarios to track the progression of these VAs. Although the features are instantaneous, we do not get the data a priori in real world scenarios to compute the HS and derive the instantaneous features and hence a short sliding window was necessary to get the time update of the instantaneous features.

\subsubsection{Constructing the Hilbert Energy Spectrum}

The IMFs for every ECG segment were decomposed using EMD. The details of the EMD algorithm may be recalled from Section 3.1.2 of the previous chapter. As mentioned earlier, only the top 7 IMFs with the highest energy were used from each segment. This was done so that every signal being analyzed had the same number of IMFs in its set in order for the comparison between them to be uniform. Since the vast majority of the signal's energy was concentrated in the first few IMFs, the information potentially discarded was negligible. These top 7 IMFs were then used to construct the HS. The instantaneous amplitudes and instantaneous frequencies of each IMF were calculated, and used to generate the HS according to Equation 2.8. The frequency range was from 0 to $\frac{F s}{2}(125 \mathrm{~Hz})$ and the frequency resolution was $0.122 \mathrm{~Hz}$, generating 1024 rows in the frequency domain. Once the HS was constructed, the instantaneous TF features could be extracted, which is explained in the following sub-section.

In the short-term analysis, the HS was constructed for each segment of the total VA signals. The first 4-s of each ECG segment were used to construct the initial HS. Subsequently, the ECG segment was expanded over a sliding window, making the ECG segment progressively longer. Each time, the HS was recalculated and used for the further extraction of instantaneous TF features. The same specifications for the HS were used for long-term and short-term analysis. 


\subsubsection{Feature Extraction}

\section{Extracting Instantaneous Features}

From the TF plane of the HS, the instantaneous TF features were extracted. The first was the $I F$, which was extracted by the moment method defined in Equation 2.9. The frequency range of the IF was chosen from 0 to Fs/8 $(31.25) \mathrm{Hz}$, and the resolution was $0.122 \mathrm{~Hz}$. This range was chosen based on the pre-processing stage, in which the ECG signals were band-pass filtered from 0.3 $\mathrm{Hz}$ to $30 \mathrm{~Hz}$. It was also known the the frequency of VF will never exceed $30 \mathrm{~Hz}$. The second instantaneous feature extracted was the $I B^{2}$, which was also extracted by the moment method described in Equation 2.10. The same frequency range and resolution was used for the $I B^{2}$ as the $I F$.

The other instantaneous feature that was extracted separately from the HS was the $I E$. For the long-term analysis, the $I E$ was calculated from the the sum of the top 2 most energetic IMFs extracted from each of the normalized and filtered ECG segments. The IE was computed by taking the square of the magnitude of the analytic IMFs, according to Equation 2.11. The instantaneous energy was hereby denoted as $I E_{12}$ due to its construction. The equation for the calculation of $I E_{12}$ is given as:

$$
I E_{12}=\sum_{j=1}^{2}\left|z_{j}(n)\right|^{2}
$$

In Equation 4.1, the $z_{j}(n)$ is analytic form of the $j^{t h}$ IMF, sorted from highest to lowest energy. Combinations of the IMFs were experimented with to compute the $I E$, but the best results for the separation between the VA groups was obtained by using the top 2 IMFs. Within those top 2 IMFs, most of the signal's energy is concentrated. The $I E_{12}$ was similarly used to observe the dynamic changes of the VAs over time. Figure 4.2 shows three sample VA segments with their instantaneous TF features pictured below them. The $I E_{12}$ was similarly used to observe the dynamic changes of the VAs over time. From these instantaneous features, statistical measures were calculated in order to quantify the behaviour of these time-varying features. That is explained in the following sub-section. For the VA trend analysis, the $I E_{12}$ was used along with the IF and $I B^{2}$ to score each 

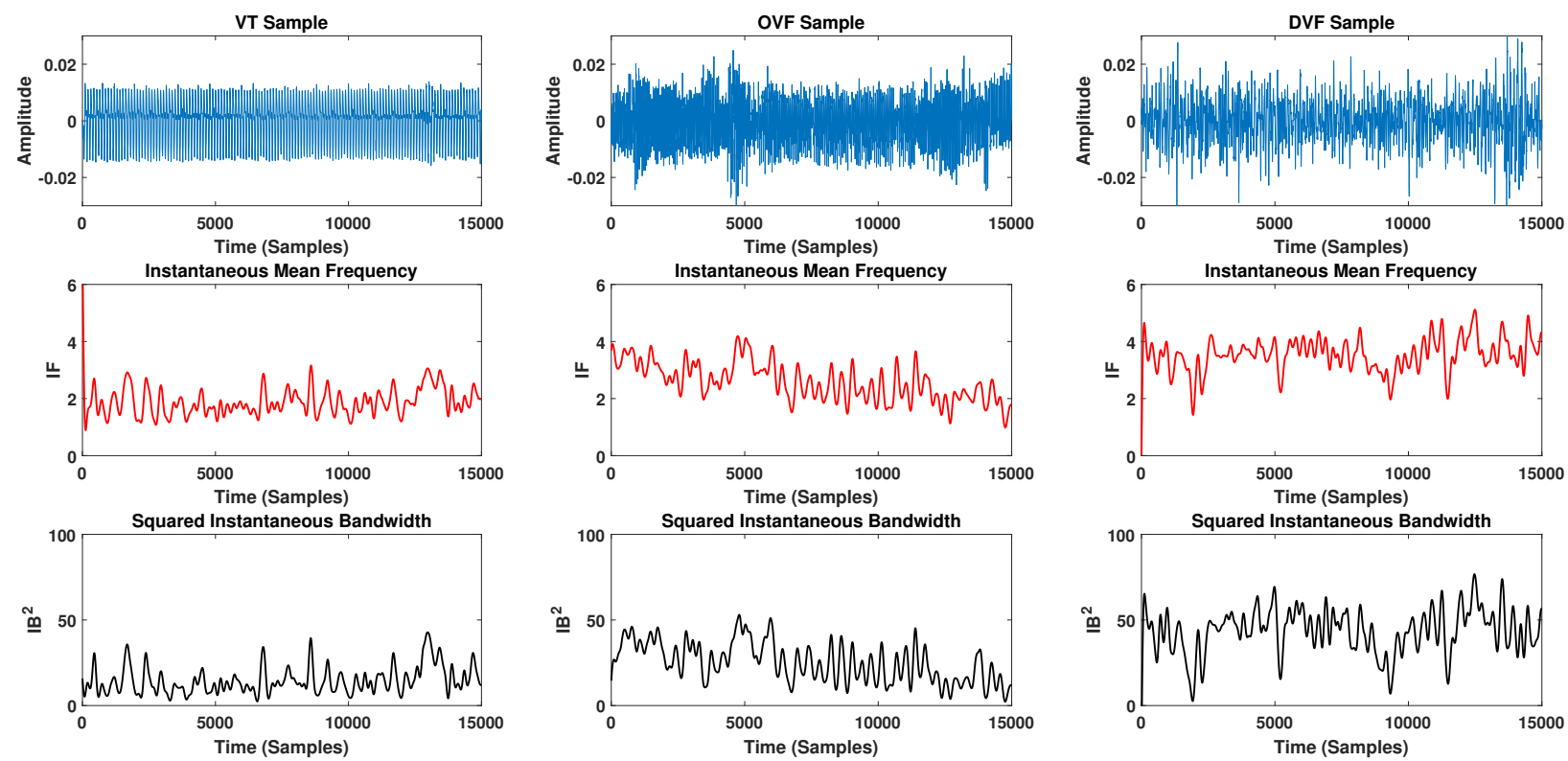

Figure 4.2: Three sample VA segments (VT, OVF, and DVF) with their extracted IF and $I B^{2}$ pictured underneath them. The IF and $I B^{2}$ were low-pass filtered for better visual illustration.

VA segment and create a trend over time. This will be further explained in a later section.

\section{Statistical Measures}

From the $I F, I B^{2}$ and $I E_{12}$, statistical measures were calculated in order to quantify the changes occurring in those features over time. These statistical measures will aid in highlighting any differences in the time-varying behaviour of the 3 VA groups. Some statistics that were calculated from the 3 instantaneous features were: mean, median, variance, RMS-value and slope. These computed quantities were used used to train the pattern classifier. The equations to calculate these features are shown below: The mean of the IF was computed as:

$$
I F_{\text {mean }}=\frac{1}{N} \sum_{j=1}^{N} I F(j)
$$

The median of the IF was calculated as:

$$
I F_{\text {median }}=I F_{\text {sorted }}\left(\frac{n+1}{2}\right)
$$

Where $I F_{\text {sorted }}(n)$ is the IF values arranged from lowest to highest. 
The variance of the IF was computed as:

$$
I F_{\text {variance }}=\frac{1}{N-1} \sum_{j=1}^{N}\left(I F(j)-I F_{\text {mean }}\right)^{2}
$$

The RMS-value of IF was calculated as:

$$
I F_{R M S}=\sqrt{\frac{1}{N} \sum_{j=1}^{N}\left[I F(j)^{2}\right]}
$$

The slope of the IF was computed as:

$$
I F_{\text {slope }}=\frac{I F(N)-I F(1)}{t_{N}-t_{1}}
$$

Similarly, the above statistical measures were extracted for $I B^{2}$ and $I E_{12}$.

In order to better observe the changes that were occurring in the $I F$ and $I B^{2}$, their gradients were also computed. By taking the gradient of the instantaneous TF features, it magnifies any rapid changes occurring over its time span. The gradient of IF and $I B^{2}$ were calculated using the central difference. The equation used to compute the gradient of IF is as follows:

$$
\text { Gradient }_{I F}(j)=\frac{1}{2}(I F(j+1)-I F(j-1))
$$

Where IF(j) is the $j^{\text {th }}$ time sample in IF. This is repeated for every value of IF. The edge values of 1 and $\mathrm{N}$ were computed by single-sided differences. This formula was used identically to calculate the gradient of $I B^{2}$.

Computing the gradient makes it easier to visualize and quantify differences in the amount of variability in these instantaneous TF features, which may prove useful for discriminating the 3 VAs. The behaviour of the gradients of IF and $I B^{2}$ were then quantified by extracting statistical measures, similar to the procedure for the original instantaneous TF features. The statistical measures extracted from the gradients were: mean and median. Additionally, the IF and $I B^{2}$ signals were also low-passed filtered ( $10 \mathrm{~Hz}$ cut-off) to remove high frequency artifacts before the gradient was computed. This was done to provide a better characterization of the true changes occurring in the instantaneous TF features. 


\subsubsection{Pattern Classification}

Similar to the previous chapter, a LDA-based pattern classifier with LOOCV was used to discriminate between the three types of VAs. The identical two stage binary classification scheme as in Chapter 3 was used here, where in the first stage VT was classified from the combined VF group, and secondly the correctly classified VF group samples were sub-classified into OVF and DVF. The LDA-based classifier was trained using statistical measures derived from the instantaneous features as described above.

\subsubsection{Scoring Index Calculation}

In order to implement the proposed method in a real world scenario we developed a scoring method for trend analysis. Time-trend analysis has many previous applications in financial and seismic analysis [64]. The scoring index will track the VA label over windowed segments and monitor the progression in the state of the arrhythmia over time. This will allow for a quantification of the trends observed in the condition of the patient. It should be noted that this is different from the short-term window analysis presented in Chapter 3. In Chapter 3 for each of the window of 4-s, only one value of feature was computed i.e. all the samples in the 4-s were used to get one value for each feature, In contrast to this chapter, the instantaneous features can be extracted for each time sample within an expanding window and quantify them over time as needed.

The procedure for the computing scoring index will now be explained. Firstly, thresholds for the IF, $I B^{2}$, and $I E_{12}$ were computed to quantify the overall spectral and temporal behaviour of the VAs. These thresholds will be used by the scoring index calculation to track the label of the VAs over every windowed segment. For every 60 -s VA segment in the database, the IF, $I B^{2}$ and $I E_{12}$ features were extracted over their total length, and the median values for each feature were computed. For each of the 3 VA groups, the mean of those median values for each instantaneous feature was calculated to determine the threshold. Any outliers present in the median groups were removed prior to the mean being taken. This helped to ensure that the thresholds computed were more accurately representative of the true group median. This resulted in unique thresholds for 
IF, $I B^{2}$, and $I E_{12}$ for the VT, OVF, and DVF groups. The thresholds were then used to create mid-points between the three VA classes. These mid-points were used to assess the scoring index for each of the windowed segments, which will be explained further below.

The 60-s VA segments were windowed in order to analyze their trend in near real-time. The initial window size was 4-s (1000 samples). On that initial 4-s, the HS via EMD was computed and the three instantaneous features were calculated. In the subsequent window, the the initial 4-s window was expanded by a sliding width of 100 samples. The HS and the instantaneous features were re-computed, and this process continued until the entire 60-s signal was analyzed. In total, there were 140 segments generated by this sliding expanding window. By using 100-sample sliding width, the analysis was able to be as computationally efficient as possible while still gathering enough information regarding the minute changes occurring in the trends over time. Please note the time update happens progressively in a real world scenario i.e., the update is done for every acquisition of 100 samples in real world which enables the near-real time feedback. The reason for expanding window approach is, since EMD is a data driven approach the addition of new data (i.e. 100 samples) might minimally influence the IMF distributions which will affect the $I E_{12}$ feature, so we recompute this on the new length of data every time. The IF and $I B^{2}$ features will not be affected as these are computed from the HS which is formed by the combination of IMFs. The updates can be stopped and restarted at pre-defined intervals to avoid elongated lengths of analysis to speed up the processing and feedback.

To score each of the windowed segments, their median values for IF, $I B^{2}$ and $I E_{12}$ were computed and compared to the median mid-points for each of the respective instantaneous features. The set of rules devised to score the VA segments were based on previous knowledge of how the VAs ought to relate to one another. It was expected that the mean frequency for the VT group would be lower compared to the OVF group, and the OVF group's mean frequency would be lower than the DVF group. It was also expected that the spectral spread for the VT group would be lowest, followed by the OVF group, and that DVF would have the highest amount. Lastly, for the 
$I E_{12}$, it was expected that VT would have the highest median energy, followed by OVF then DVF. This is because the distribution of energy is expected to be the most uniform in VT, less uniform in OVF, and the most variability in DVF. The set of rules for scoring the segments based on the three instantaneous features is described below.

\section{IF:}

1. If the median IF of the segment was less than or equal to the mid-point between the VT and OVF thresholds $\left(\left(V T_{I F_{t h}}+O V F_{I F_{t h}}\right) / 2\right)$, then score it as VT (0).

2. IF the median IF of the segment was greater than $\left(\left(V T_{I F_{t h}}+O V F_{I F_{t h}}\right) / 2\right)$ but less or equal to the mid-point between the OVF and DVF thresholds $\left(\left(O V F_{I F_{t h}}+D V F_{I F_{t h}}\right) / 2\right)$, then score it as OVF (1).

3. Otherwise, score the segment was DVF (2).

The set of rules were the same for $I B^{2}$. However, the rules for scoring $I E_{12}$ were reversed, since median energy was expected to be highest for VT. Based on the resulting scores for each windowed segment from the three instantaneous features, the mode score was identified and was chosen as the final score for that segment. For example, if two of the instantaneous features scored the segment as 0 (VT), and the other one scored it as 1 (OVF), then ultimately the segment would be scored as 0 (VT). Doing this would ensure that all three instantaneous features had input into the final score, and that the score would be less influenced by extraneous results from one of the features.

In order to provide feedback on the evolution of the arrhythmia over time, a cumulative averaging strategy was implemented. This was done by taking the previous segment's score and averaging it with the current segment's score to produce the next score. This allowed for us to get a sense of how the scores are changing relative to each other, instead of each windowed segment's score being independent. The equation for how the cumulative averaging was computed is shown below:

$$
\operatorname{Score}(m)=\frac{\operatorname{Score}(m)+\operatorname{Score}(m-1)}{2}
$$

In equation 4.8, $\operatorname{Score}(m)$ represents the modal score generated from the three instantaneous 
features for the current $m^{\text {th }}$ windowed segment, and $\operatorname{Score}(m-1)$ is the score from the previous segment. By computing the cumulative scores for the windowed segments over the entire duration of each 60-s VA segment, trends regarding how the VA type was evolving over time may be seen. Observing these trends would provide us with dynamic feedback as to how the VA is progressing over time. For instance, when a patient is undergoing OVF in an 'out-of-hospital' setting, the EMS personnel may choose to use pacing to control the arrhythmia and bring it back to normal rhythm. However, in the case of DVF, the only treatment option available is a defibrillation shock to attempt to reset the heart's rhythm. If the EMS personnel could receive near real-time feedback as to how the patient's VA is progressing or how they are responding to treatment, they could alter treatment options quickly to adapt to the evolving arrhythmia. This proposed method has the potential to provide such a feedback tool.

\subsection{Results}

In this section the results of this proposed approach will be presented. It will be divided in three sub-sections. The first sub-section will contain the results of the long-term analysis for classifying the VAs over their full 1-minute duration for 'in-hospital' settings. The second sub-section will present the VA trends analysis results, where the trends in the VAs were monitored over time for 'out-of-hospital' settings. Thirdly, the VA classification results for combining the long-term non-instantaneous and instantaneous features from Chapter 3 and 4 will be presented.

\subsubsection{Long-Term (In-Hospital) Analysis}

In the first stage of classification between VT and VF, there were $22 \mathrm{VT}$ signals and 39 total VF signals. When training the LDA-based classifier, the best result was achieved using a combination of four features. Those four features were: $I E_{12}$ median, $I E_{12}$ variance, gradient IF median, and IF slope. The boxplots of these features are shown below. In the boxplot in Figure 4.3a, the median of $I E_{12}$ was generally higher for the VT group compared to the VF group. The trend described the fact that the energy of VF is definitively lower compared to VT. This observation fit with our understanding that VF is a worsened condition than VT, resulting in a lessened relative ECG am- 


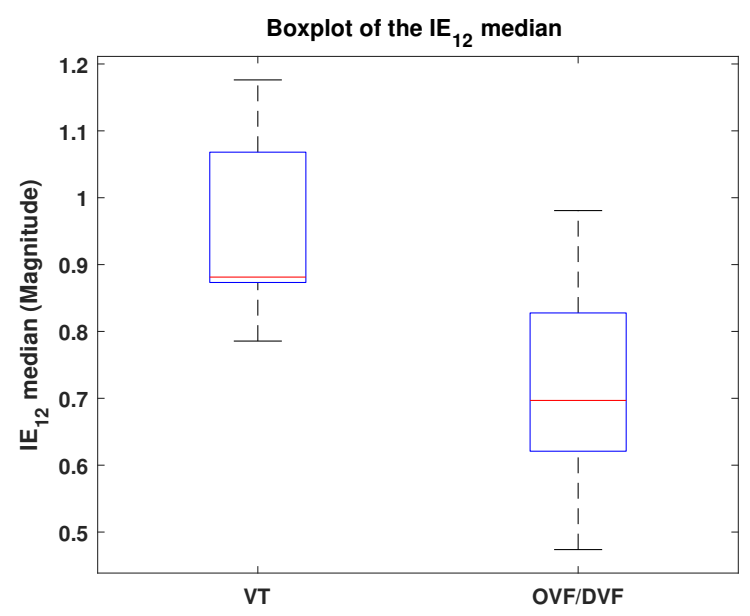

(a) $I E_{12}$ median.

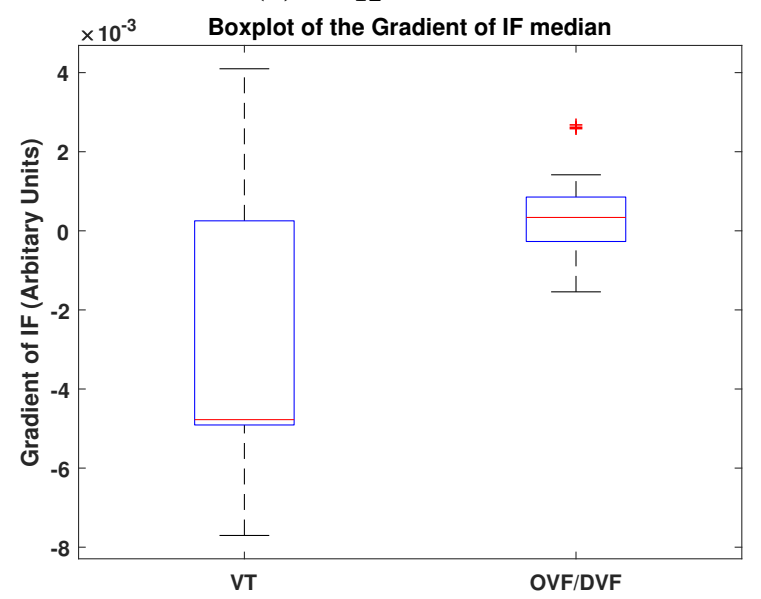

(c) Gradient IF median.

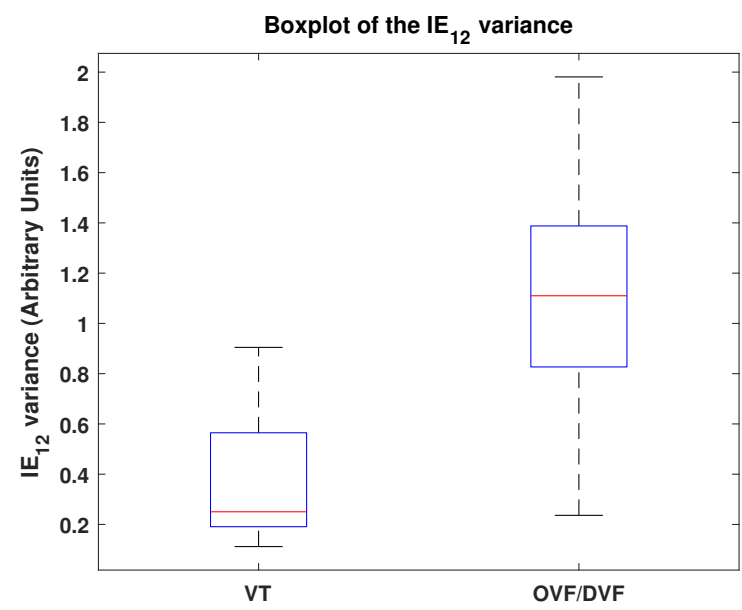

(b) $I E_{12}$ variance.

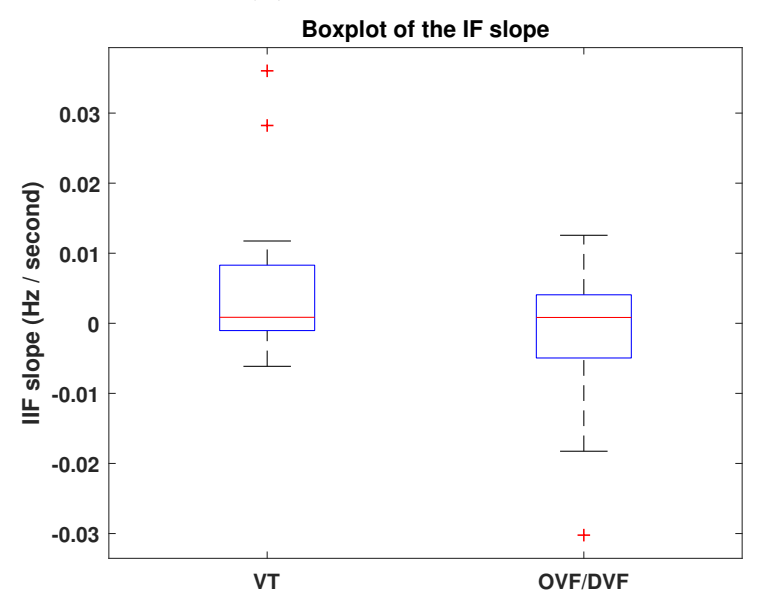

(d) IF slope.

Figure 4.3: The boxplots for the 4 features used to train the VT vs. VF classifier.

plitude.

In the boxplot for $I E_{12}$ variance in Figure 4.3b, the VF group was clearly above the VT group. The $I E_{12}$ variance indicated the amount of temporal variability present in the VA segments. It was clear that VF had a higher amount of energy volatility over time than VT, which fits with our previous understanding of these two VA types. Remember that the ECG segments were normalized to the same cumulative energy in the pre-processing stage, and thus these differences observed in energy content were not biased by differing amplitudes of the signals. 
In the third boxplot of Figure 4.3c, the gradient IF median demonstrated a similar trend. The median value was higher for VF compared to VT, indicating that VF contained larger spectral changes over time than VT. Since it is well understood that VT and VF can be separated by spectral measures, this result was consistent.

Lastly, the boxplot for IF slope was shown in Figure 4.3d. This feature was not highly discriminatory on its own, likely due to the absence of monotonic increase/decrease in the mean frequency of the VAs over time. However, this feature helped to improve the overall separation of the VT and VF groups when used in combination with the other three features above. Overall, these results indicated that spectral changes over time and the differences in instantaneous changes in energy were strong discriminating factors between the VT and VF groups.

The total cross-validated accuracy achieved in Stage 1 was $96.7 \%$. Within the VT group, 1 out of 22 samples were misclassified, and 1 of the $39 \mathrm{VF}$ samples was misclassified. The confusion table summarizing the results in shown in Table 4.1. The ROC curve for the stage 1 classifier is shown in Figure 4.4.

Table 4.1: VT vs. VF classification accuracy for instantaneous features using leave-one-out crossvalidated data.

\begin{tabular}{|c|c|c|c|c|}
\hline Method & Groups & VT & VF & Total \\
\hline Cross-validated & VT & $\mathbf{2 1}$ & 1 & 22 \\
\hline & VF & 1 & $\mathbf{3 8}$ & 39 \\
\hline$\%$ & VT & $\mathbf{9 5 . 5}$ & 4.5 & 100 \\
\hline & VF & 2.6 & $\mathbf{9 7 . 4}$ & 100 \\
\hline
\end{tabular}

The ROC curve's global pattern remained well above the diagonal bisector, which demonstrated a strong classifier performance. This strong performance was further quantified by the AUC, which had a value of 0.995 . The p-value for linear discriminant scores of the overall classifier was significant $(p<0.01)$. Overall, it appeared that the inclusion of the frequency based features (IF and $I B^{2}$ ) helped to improve the separation of VT and VF when compared to Chapter 3. Although not as challenging as classifying OVF and DVF, the problem of separating PVT and 
OVF was still addressed here. PVT and OVF are highly similar in their temporal complexity, and this classifier was able to discriminate them effectively.

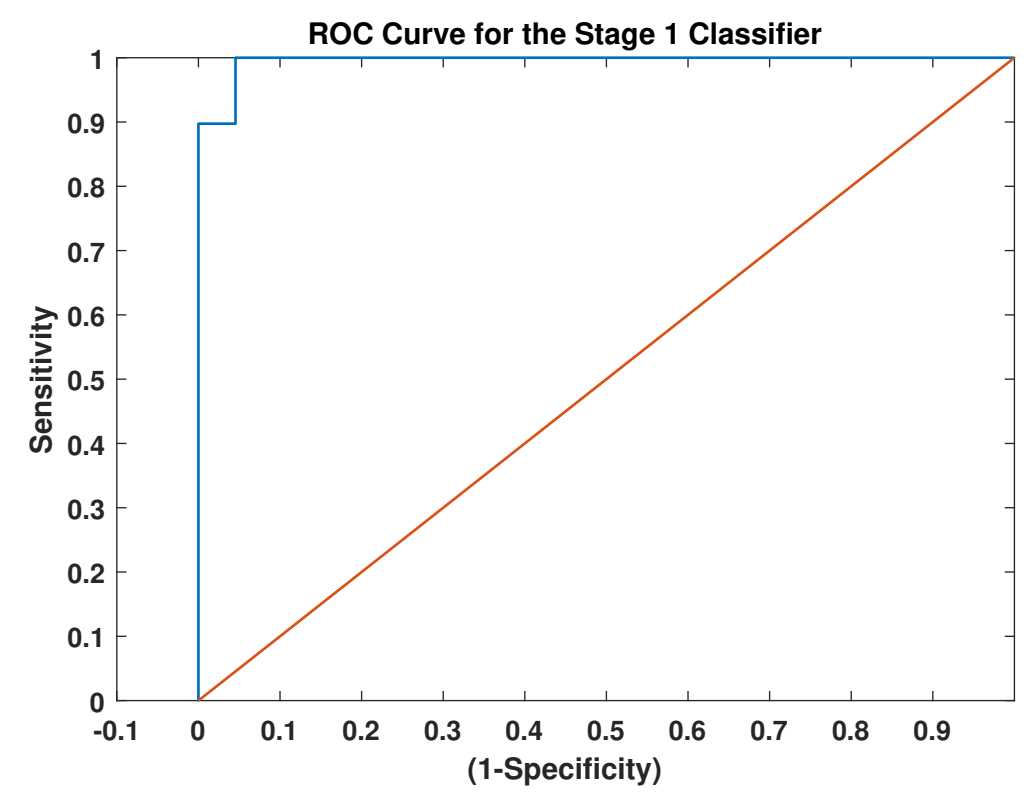

Figure 4.4: The ROC curve for the VT vs. VF classifier.

In the second stage of the binary classification scheme, the single misclassified OVF sample was removed. There were 16 OVF and 22 DVF samples used in this stage. The LDA-based classifier was trained using the following four features to obtain the maximum accuracy: $I E_{12}$ median, gradient IF variance, IF variance, and $I B^{2}$ variance. The boxplots for these four features are shown below.

In the boxplot for the $I E_{12}$ median in Figure 4.5a, it was shown that the OVF group again had a higher value compared to the DVF group. This demonstrated that the average amount of energy over time is in general higher for OVF than DVF. As DVF tends to devolve from OVF, the condition of the patient's heart is worsening which may be resulting in a lower magnitude in the ECG recording. In Figure 4.5b, it was apparent that the DVF group was higher than the OVF group. The gradient IF variance features quantifies the amount of variability present in the spectral differences over time. Thus, it was shown that the spectral complexity of DVF was greater than OVF. Thirdly, 


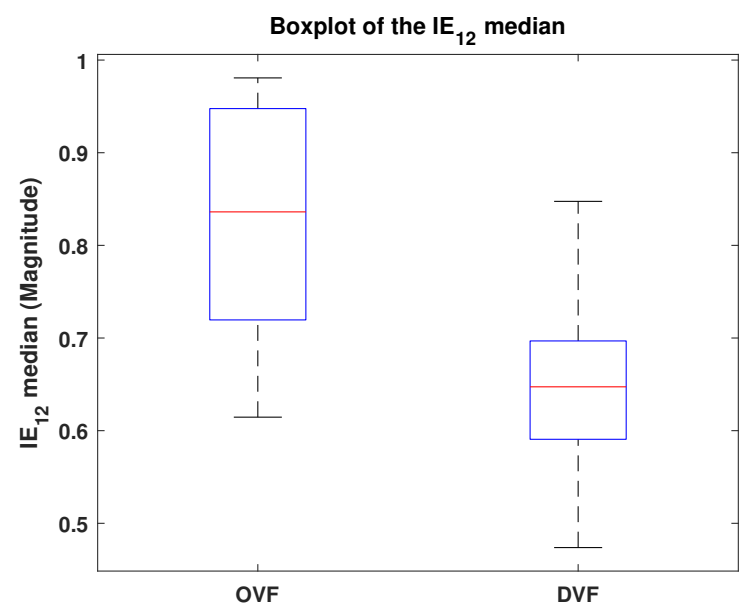

(a) $I E_{12}$ median.

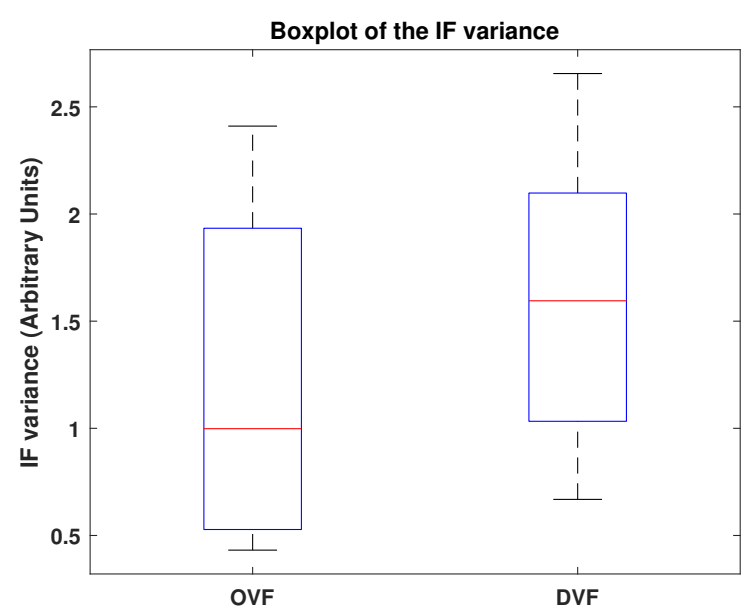

(c) IF variance.

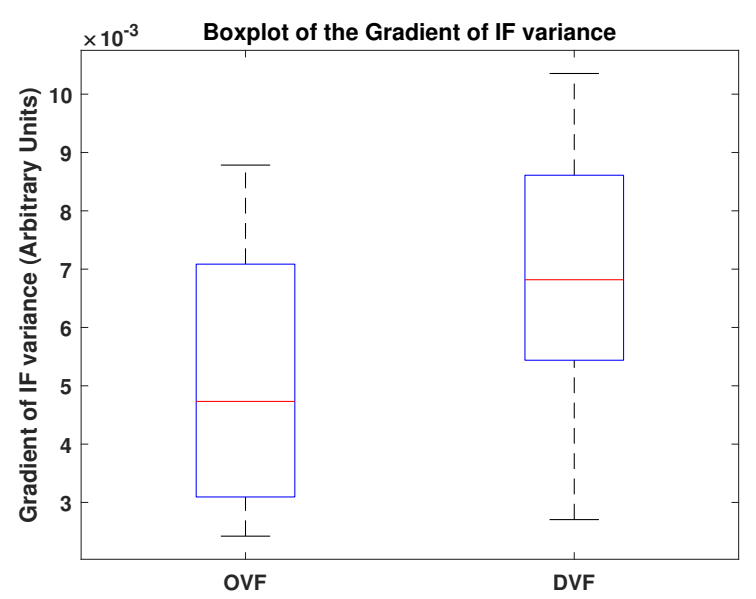

(b) Gradient IF variance.

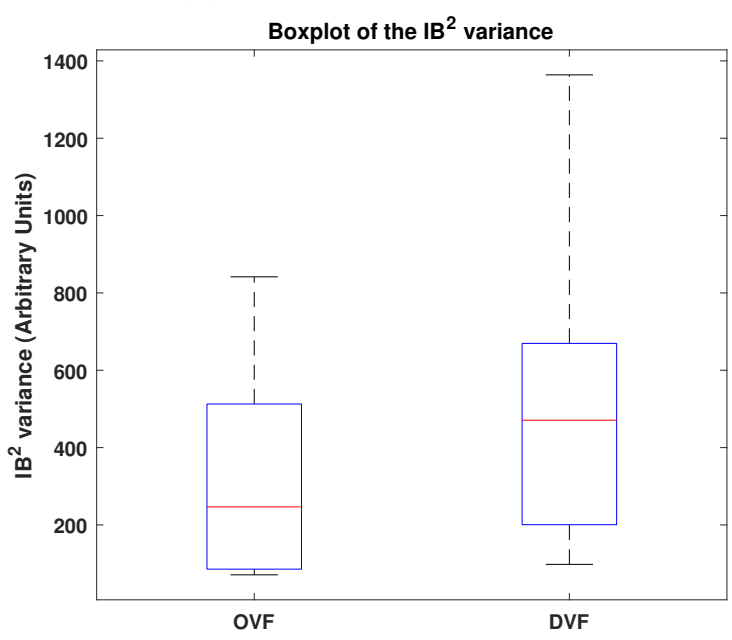

(d) $I B^{2}$ variance.

Figure 4.5: The boxplots for the 4 features used to train the OVF vs. DVF classifier.

in the boxplot for IF variance in Figure 4.5c, there was a slight difference between the two groups. This features indicated the level of variability in the mean frequency over time. Lastly, for the $I B^{2}$ variance in Figure 4.5d, there was a subtle difference visible in the boxplots of OVF and DVF. This feature helped to quantify the difference in temporal complexity between OVF and DVF. Although not highly discriminatory, when combined with the other features, IF variance and $I B^{2}$ variance helped to improve the overall separation between OVF and DVF. These results demonstrated that spectral and energy differences over time were effective at separating these two sub-classes of VF. 
The LOO cross-validated accuracy obtained in Stage 2 was $81 \%$. The summary of the classification results can be seen in Table 4.2. In total, there were 4 out of 16 OVF samples misclassified and 3 out of 22 DVF samples misclassified. In Figure 4.6 the ROC curve for the stage 2 classifier is shown. The global pattern of the curve demonstrated a strong discriminating power for the this classifier. The AUC for this ROC curve was 0.935, which further emphasizes the strong performance. The combined the p-value for discriminant scores of the total classifier was significant $(p<0.01)$. These measures validate the strong performance achieved by the stage 2 classifier. The relative drop in performance from stage 1 to stage 2 was expected, since the the classification task in stage 2 was a more difficult, yet more pertinent to resuscitation success.

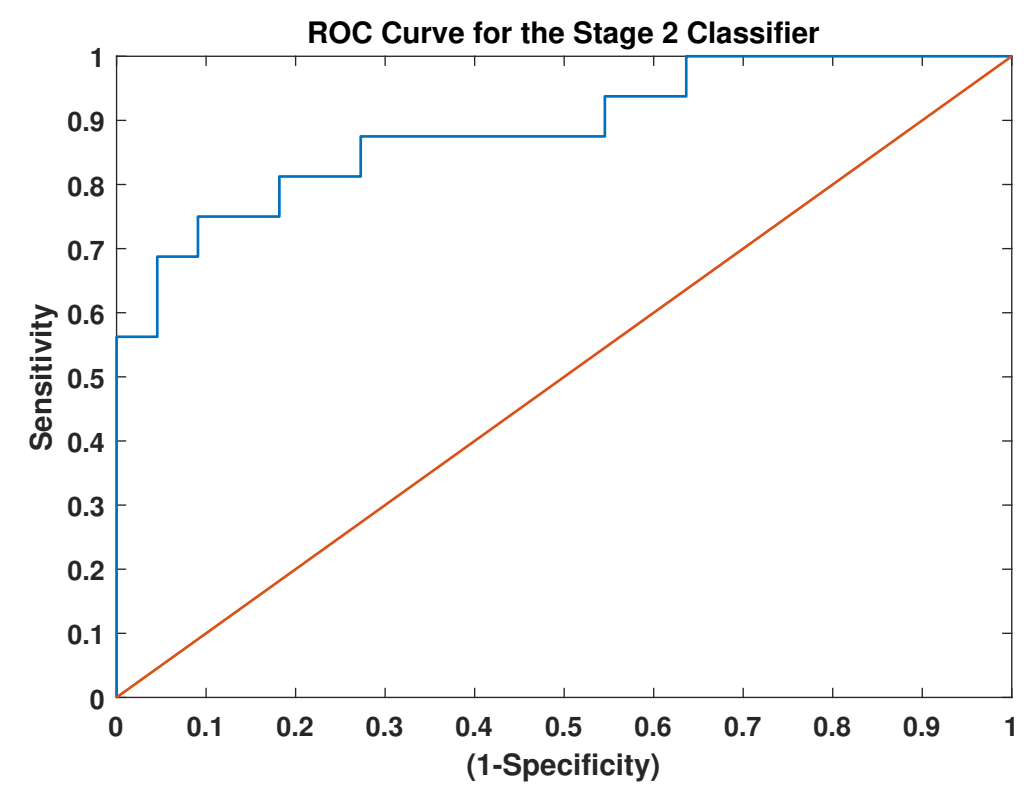

Figure 4.6: The ROC curve for the OVF vs. DVF classifier.

Table 4.2: OVF vs. DVF classification accuracy for instantaneous features using leave-one-out cross-validated data.

\begin{tabular}{|c|c|c|c|c|}
\hline Method & Groups & OVF & DVF & Total \\
\hline Cross-validated & OVF & $\mathbf{1 2}$ & 4 & 16 \\
\hline & DVF & 3 & $\mathbf{1 9}$ & 22 \\
\hline$\%$ & OVF & $\mathbf{7 5 . 0}$ & 25.0 & 100 \\
\hline & DVF & 13.6 & $\mathbf{8 6 . 4}$ & 100 \\
\hline
\end{tabular}


There was no comparative analysis performed for this part of the results. This was because there were no comparative features that could be extracted instantaneously like IF, $I B^{2}$, and $I E_{12}$. The DF, EN, SE and other static features all require a pre-allocated amount of data before they can be extracted. For this reason, the comparative analysis was not included.

\subsubsection{VA Trends Analysis}

The results for the VA trends analysis will now be presented. The scoring index calculation was used to label each windowed segment of the VA signals, and cumulative averaging was performed to obtain the overall trend in the VAs over time. The median thresholds for the IF were: 1.57 $\mathrm{Hz}$ for VT, $1.9 \mathrm{~Hz}$ for OVF, and $2.41 \mathrm{~Hz}$ for the DVF group. The median thresholds for the $I B^{2}$ thresholds were: $3.62 \mathrm{~Hz}^{2}$ for VT, $4.86 \mathrm{~Hz}^{2}$ for OVF, and $8.73 \mathrm{~Hz}^{2}$ for DVF. Lastly for $I E_{12}$ the median thresholds were: 0.874 for VT, 0.829 for OVF, and 0.646 for DVF. These thresholds were used to score the VA segments according to the scoring index calculation rules described above.

We will present one case that illustrates the potential that this approach has for near real-time feedback in emergency settings. Figure 4.7 shows another example case of the changing trend for a VF sample. This VF sample was labeled as a 'mostly' DVF sample in our database. In the initial part of the segment, the VA trend was bordering between VT and OVF. Judging by the signal characteristics of the first 15 seconds, the segment appeared to be PVT due to its relatively stable frequency and minimal amplitude variations as seen in the zoomed-in portion. From 15 seconds to 30 seconds, the VA trend was scoring the segment as OVF as the signal becoming more disorganized. The signal then trended toward DVF, with a brief drop back into VT before continuing as mostly DVF from 50 seconds onward. It was visible within the ECG signal's characteristics that in the latter stages the signal was much more disorganized, which was characteristic of DVF.

The significance of the above result was that a marked progression in the VA segment was visible over time. The VA segment started off as a PVT, then it progressed more into OVF, and lastly it devolved into DVF. This type of dynamic trend information may be extremely useful to EMS 

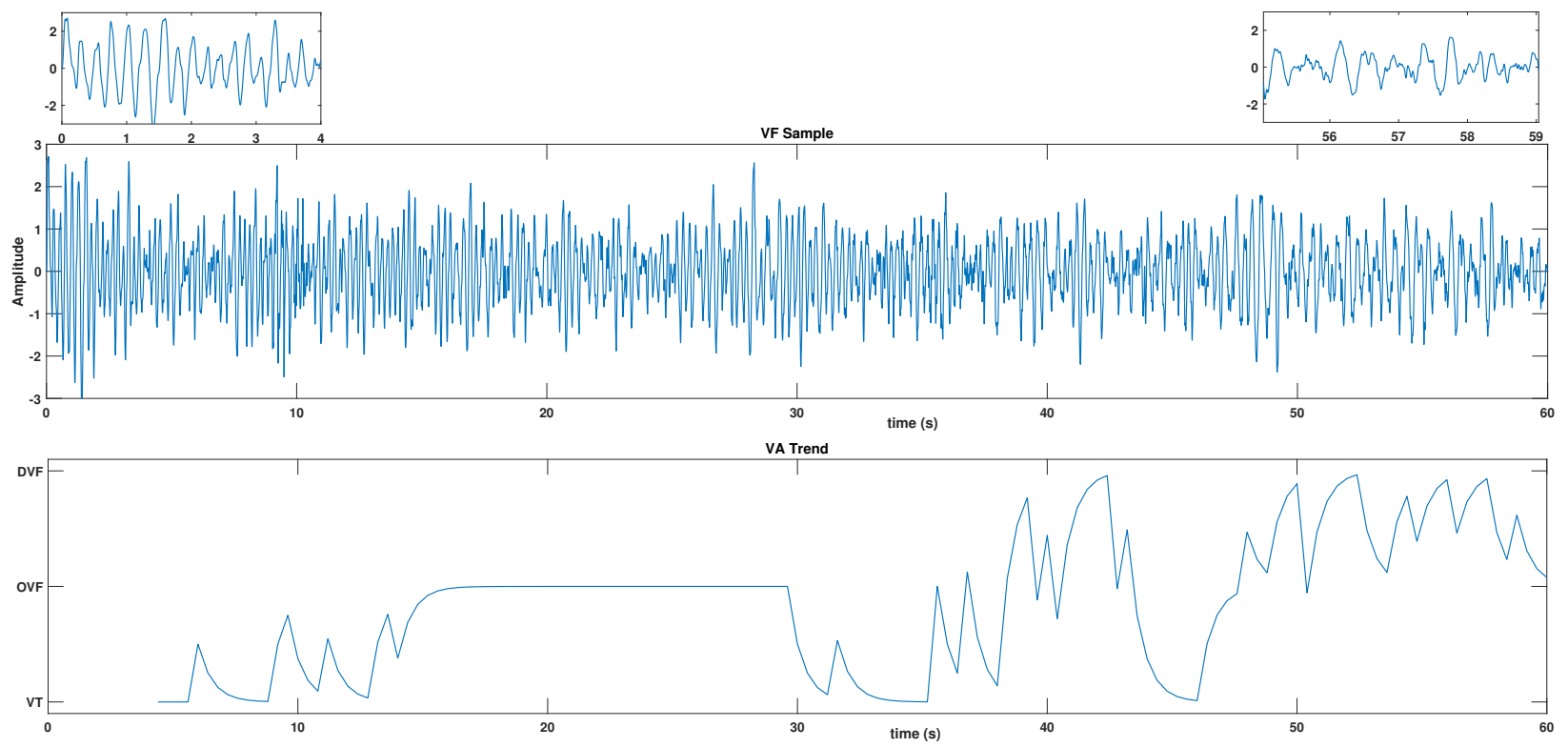

Figure 4.7: The plot of a VF sample along with its VA trend signal shown below.

personnel while treating a patient. By looking at the VA trend over time, EMS could observe that the patient's VA is getting progressively worse over time, to the point that it becomes DVF. At that point, the medical staff would be informed that treatment needs to be modified and defibrillation shock is required immediately to revive the patient. The near immediate feedback that EMS receives could lead to time saved during the 'out-of-hospital' scenario trying to diagnose the patient. Due to the time sensitivity of these VAs, helping the EMS personnel monitor changes in the VA condition could hopefully improve the patient's chances of survival.

\subsubsection{Long-Term Analysis Combining Non-Instantaneous and Instantaneous Features}

In the final part of this chapter, the near real-time and instantaneous features used for the longterm analysis in Chapters 3 and 4 will be combined to test the classification accuracy for 'inhospital'/offline analysis. To test the results, the same two-stage binary classification scheme was performed, initially separating VT from VF and subsequently sub-classifying OVF and DVF. In the initial stage for classifying VT and VF, there were 22 VT and 39 total DVF segments. The 
classifier was trained with the following 5 features to obtain the best result: $I E_{12}$ variance, $I E_{12}$ median, gradient IF median, ERV slope (4s) and ERS variance (1s).

The total classification accuracy achieved was $96.7 \%$. A summary of the results is shown in Table 4.3. Overall, 2 out of the 22 VT segments were mis-classified, but none of the VF segments were mis-classified. The ROC curve for this classifier is also pictured in Figure 4.8. It was shown in Figure 4.8 that the curve remained well above the diagonal bisector, indicating a strong performance. The AUC for this curve was equal to 0.993, further validating the strong discriminating power. The $\mathrm{p}$-value of the linear discriminant scores was also significant $(p<0.01)$. The result for this classifier improved upon the results obtained using just instantaneous features for classifying the VF segments. The high classification accuracy achieved is well explained by the statistical measures mentioned, and is in alignment with previous works.

In the second stage of classification where OVF was classified from DVF, there were 17 OVF and 22 DVF segments used. The highest classification accuracy was achieved when the classifier was trained with the following five features: $I B^{2}$ slope, IF gradient median, $I E_{12}$ mean, ERS total, and ERV variance $(4 \mathrm{~s})$. The classification accuracy achieved in this stage was $87.2 \%$. The summary of the classification results is shown in Table 4.4. In total, 2 of the 15 OVF segments and 3 of the 22 DVF segments were misclassified.

The ROC curve for the OVF vs. DVF classifier is depicted in Figure 4.9. The global pattern of the curve indicated a strong discriminatory ability. The AUC for the curve was 0.968 , which

Table 4.3: VT vs. VF classification accuracy for combined features with leave-one-out crossvalidated data.

\begin{tabular}{|c|c|c|c|c|}
\hline Method & Groups & VT & VF & Total \\
\hline Cross-validated & VT & $\mathbf{2 0}$ & 2 & 22 \\
\hline & VF & 0 & $\mathbf{3 9}$ & 39 \\
\hline$\%$ & VT & $\mathbf{9 0 . 9}$ & 9.1 & 100 \\
\hline & VF & 0 & $\mathbf{1 0 0}$ & 100 \\
\hline
\end{tabular}




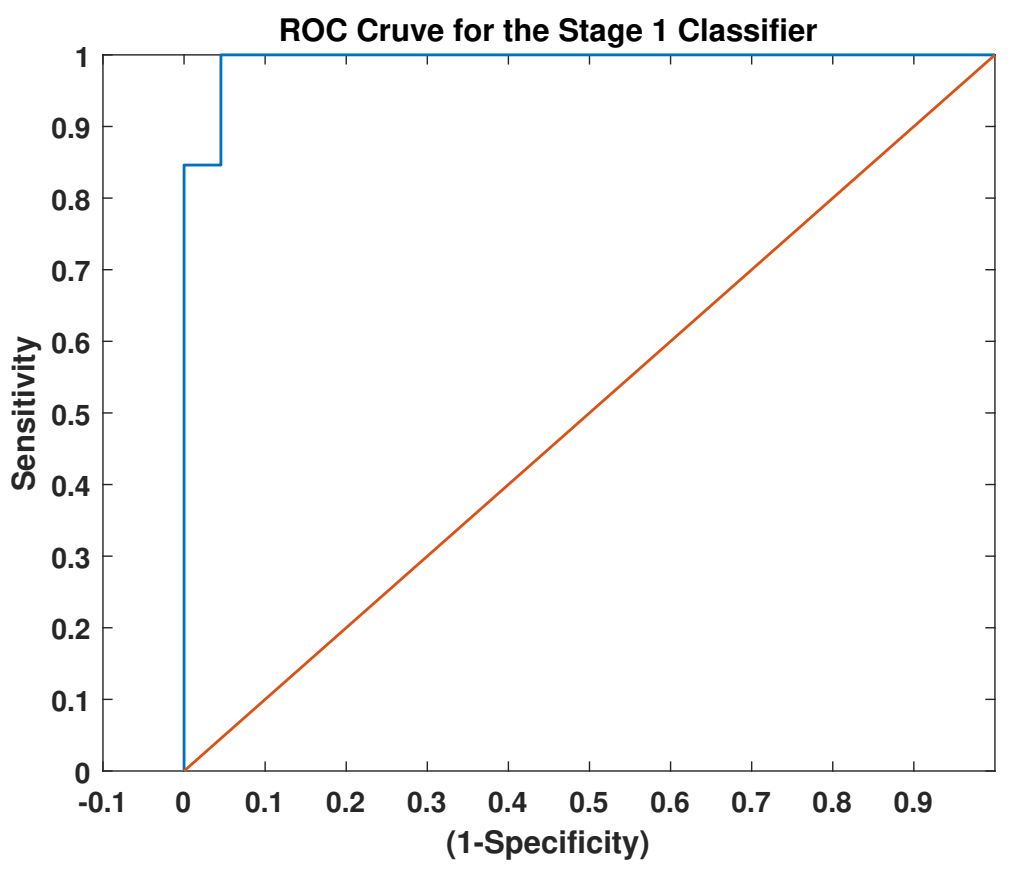

Figure 4.8: The ROC curve for the combined VT vs. VF classifier.

further explained the strong classification performance. The overall p-value for the linear discriminant scores was significant $(p<0.01)$.

There were two frequency-based features used to train this classifier: $I B^{2}$ slope, and gradient IF median. These features helped to quantify the subtle differences in spectral behaviour between the OVF and DVF groups. The $I E_{12}$ mean was an energy based feature that helped to quantify the differences in mean energy over time between OVF and DVF. Lastly, the IMF-energy based features of ERS total and ERV variance (4s) also helped to separate the two groups. These features helped to quantify the temporal complexity between OVF and DVF, which is known as a strong

Table 4.4: OVF vs. DVF classification accuracy for combined features with leave-one-out crossvalidated data.

\begin{tabular}{|c|c|c|c|c|}
\hline Method & Groups & OVF & DVF & Total \\
\hline Cross-validated & OVF & $\mathbf{1 5}$ & 2 & 17 \\
\hline & DVF & 3 & $\mathbf{1 9}$ & 22 \\
\hline$\%$ & OVF & $\mathbf{8 8 . 2}$ & 11.8 & 100 \\
\hline & DVF & 13.6 & $\mathbf{8 6 . 4}$ & 100 \\
\hline
\end{tabular}




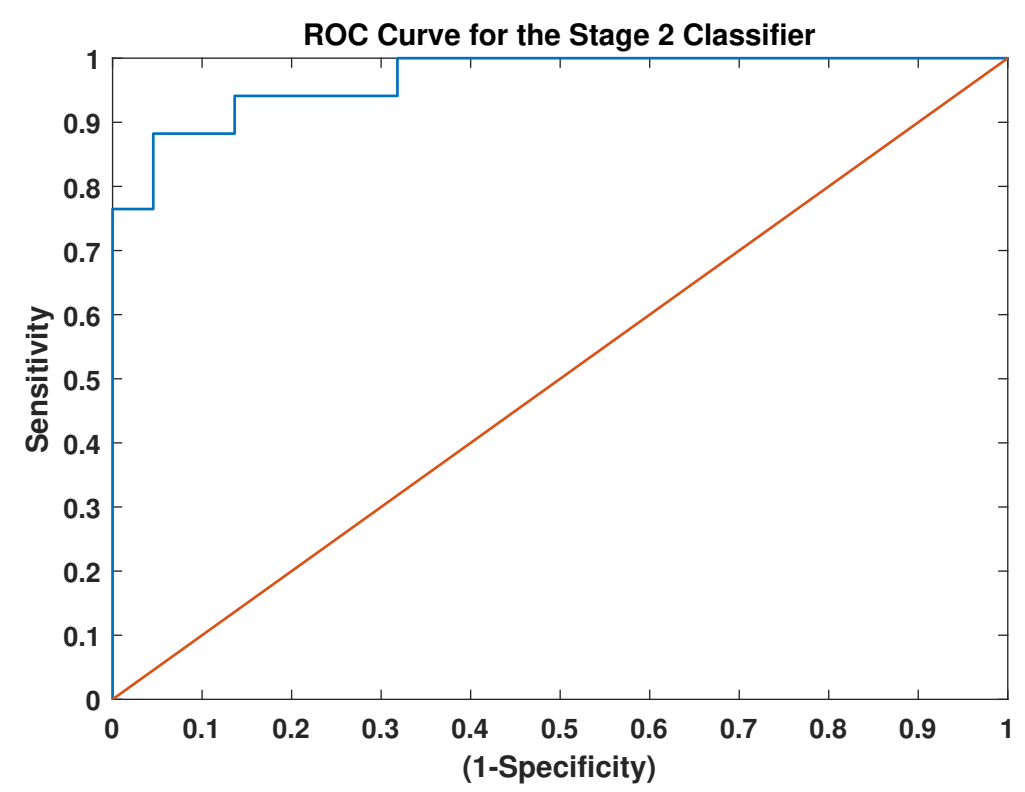

Figure 4.9: The ROC curve for the combined OVF vs. DVF classifier.

discriminating factor between the two groups. Together, this group of features was able to improve upon classification accuracy between OVF and DVF achieved in the previous two long-term analysis approaches presented. Comparing this result to the OVF vs. DVF classification accuracy achieved by comparable previous works, this result was able to exceed them all. This approach also comes with the additional benefits of being completely data-driven and computationally efficient to implement. These same features may be applied to any VA database without the need to choose a basis function. Overall, the results of this analysis showed promising results in tackling a challenging problem of separating OVF and DVF, and may be useful to doctors in planning treatment options for 'in-hospital' settings.

\subsection{Discussion}

Using the instantaneous features described in this chapter, a long-term (in-hospital) analysis approach was devised to classify the 3 types of VAs. The classification accuracy for the first stage of VT vs. VF was $96.7 \%$, and the for the second stage of OVF vs. DVF it was $81 \%$. Compared to the results of Chapter 3 regarding long-term analysis, this proposed approach made improvements to both classification stages. In the previous chapter, the extracted features were IMF-energy based 
features that quantified the temporal complexity of the VA segments. That made those features suitable to classifying OVF and DVF, but less ideal for VT and VF. It is well-known that VT and VF may be accurately separated by frequency parameters, and in this chapter the IF and $I B^{2}$ features helped in that classification. As for OVF vs. DVF, the instantaneous features improved marginally on the results of Chapter 3. However, in Chapter 3 it only required a single feature (ERS median (1s)) to classify OVF and DVF effectively whereas in this approach 3 features were used. The $I E_{12}$ median was the dominant feature for classifying OVF and DVF, but the gradient IF and gradient $I B^{2}$ helped to improve the maximum classification accuracy achieved.

The instantaneous TF features were extracted from the HS, an EMD-based TF plane. The HS has some key advantages when compared to other types of TFDs. As we have discussed, EMD is a fully data-driven approach making it independent of any basis function for decomposition. Thus, the HS facilitates TF analysis while remaining unbiased by the choice of a basis function. This proposed approach could be used on any given VA database without any prior adaptation required. Another benefit of the HS is that it is quite computationally efficient to compute compared to other TFDs. The traditional TFDs like the WVD are much more computationally expensive, and thus are time-consuming for extracting instantaneous TF features. Due to the ease of implementation of the EMD algorithm and the construction of the HS, extraction of these instantaneous features can be accomplished much faster by this approach. Also, cross-terms are not an issue in the HS like they are in traditional quadratic TFDs. Lastly, the TF resolution of the HS is higher than in other TFDs. The time and frequency bin sizes can be independently controlled by the user, enabling it to achieve finer resolution than Fourier-based methods.

In the VA trends analysis portion of this chapter, the instantaneous features were used to devise a scoring method by which windowed segments of the VAs would be analyzed to track its progressions over time. This short-term analysis method utilized the strength of the instantaneous features which was their ability to monitor the progression of the VAs instantaneously. To the author's knowledge, this was the first approach that attempted to use HS-based instantaneous TF 
features to track the dynamic progression of VF over time and provide near real-time feedback on the trends observed. A sliding width of 100 samples (0.4s) was used to update the HS and instantaneous features, and cumulative averaging was used to measure the relative change between consecutive segment scores. A longer sliding width and cumulative averaging between more points may smooth the VA trend plot to provide better visual illustration for the user. However, due to the time critical nature of VF, there is a limit to how much smoothing can be done. In 'out-of-hospital' settings, introducing longer sliding windows and more cumulative averaging will increase the delay between the incoming patient ECG data and the trend analysis plot. EMS personnel need to react quickly to evolutions in VF over time, and so keeping the analysis as close to real-time as possible was paramount.

Using VA trend analysis, an example of a potential real-world scenario was explored and the virtues of this approach were demonstrated. The scenario showed a VA that was progressively worsening from PVT towards OVF and finally DVF. This scenario could occur 'out-of-hospital' when a patient collapses, and while being monitored by EMS the VA devolves into DVF. The trend analysis method was able to track the transitions occurring in the VA, and this feedback could help inform EMS/paramedics about when it is appropriate to deliver the correct treatment. Being able to observe transitions in the state of an arrhythmia would also assist medical personnel in knowing when to modify treatment options to fit with the current condition of the VA. Because of the near real-time nature of this approach, swift feedback may be given to EMS which may increase the likelihood of a patient surviving, particularly if they are suffering from VF. Also, since this VA trend could alert EMS personnel if the patient is progressing towards a more serious VA like DVF, they could begin to plan ahead and administer counter-treatments to help ensure that the patient never devolves into that lethal condition at all.

There are other potential applications for this VA trend analysis, including monitoring the effectiveness of treatment modalities. For instance, a patient may be given an anti-arrhythmic drug to help bring the heart rhythm back to more controlled state. The VA trend analysis could monitor 
how the condition of the heart is progressing over time by visualizing any changes happening in the VA type. The medical personnel could use this feedback to verify if and when these drugs have had their intended effect. Once the trend reaches the specific VA condition that is desired, the medical personnel can move on to administering further steps of treatment to return the patient's cardiac rhythm back to normal.

Short-term analysis for near real-time feedback was also performed in Chapter 3, but this trend analysis differs in some important ways. In Chapter 3, the ERS and ERV features were computed over every 4-s segment. From each segment, one value for the feature was computed, related to the global pattern present in that segment. In this approach however, the 3 instantaneous features have values for every sample present within each segment. This means that the instantaneous features can quantify local patterns occurring inside of each windowed segment. Secondly, the features extracted in Chapter 3 capture the trends occurring across the IMFs, and not in the time direction. The ERV/ERS features quantify the organizational complexity in the IMF direction, while the act of windowing the segments is what introduces the time direction to the analysis. The instantaneous features on the other hand, are inherently linked to the temporal direction since they are extracted per sample. This means that the VA trend analysis captures the changes occurring within the windows, and updates the progression as the window expands, whereas the ERV/ERS method can only capture changes occurring between windows. What the method in Chapter 3 can do that this method cannot is provide the classification of the VA at any given time. The non-instantaneous short-term analysis method may be halted or resumed at any time, and based on the buffer of data it has received so far, it can output a VA classification. The purpose of this VA trends analysis however, is not to classify VAs specifically, but to monitor the progression of the VAs over time to indicate how the arrhythmia is evolving.

Using the combined non-instantaneous and instantaneous features, the classification accuracy for VT vs. VF and OVF vs. DVF was re-examined. For the first stage of VT vs. VF, the total accuracy achieved was $96.7 \%$. This result was comparable with the results using instantaneous 
features only, except that none of the VF segments were mis-classified. This result also exceeded the results using Balasundaram's features, which achieved only 93.4\%. In this stage, a combination of $I E_{12}$ features, frequency features, and IMF-energy features were used to train the classifier. The benefits of each of these three types of features were utilized to produce a strong discrimination between VT and VF. In the secondary classification of OVF and DVF, the achieved total classification accuracy was $87.2 \%$. This result exceeded the classification accuracy of using instantaneous features only, and the result achieved by Balasundaram's features on this database. By using the combination of complexity, energy-based and spectral features, the classifier was able to successfully discriminate OVF and DVF better than any other previous work. The differences in frequency and energy over time were captured by the instantaneous features, and the differences in temporal complexity were captured by the ERV/ERS features.

Overall, this proposed approach was successfully able to address the difficult problem of discriminating OVF and DVF. Addressing this problem may lead to improved resuscitation outcomes 'in-hospital', as this approach could help clinicians to more accurately diagnose these VAs and administer the appropriate treatment options accordingly.

\subsection{Chapter Summary}

In this chapter, we presented the use of instantaneous features for performing long-term analysis of VAs 'in-hospital' and short-term trends analysis for use in 'out-of-hospital' incidents. In the long-term analysis, the instantaneous TF features IF and $I B^{2}$ were extracted from the HS, along with the $I E_{12}$ from the IMFs. The features were used in a two-stage binary classification scheme, and were able to successfully discriminate VT from VF and OVF from DVF with high accuracy. For the VA trends analysis, the instantaneous features were extracted over windowed segments of the VA signals to track the progression of the VAs over time. This method was able to monitor the progressions and transitions occurring in the VAs in near real-time, which can assist EMS in selecting and modifying treatments based on the evolving arrhythmia. Lastly, the long-term analysis features from Chapters 3 and 4 were combined to devise an improved classification scheme for the 
3 VA types. The maximum classification accuracies were improvements to the results obtained by previous methods. This proposed approach could assist clinicians 'in-hospital' to more accurately diagnose these VAs and help them to select and plan appropriate treatment modalities. 


\section{Chapter 5}

\section{Conclusions}

ARDIOVASCULAR conditions known as VAs may seriously impact the health of patients
and could even become lethal. Accurately and swiftly detecting these conditions is essential for selecting the most appropriate treatment options for patients. Since patients suffering from VF only have a few minutes to receive treatment before it becomes lethal, detection speed is also critical in improving the chances of patient survival in 'out-of-hospital' incidents. It has been shown that VAs are highly progressive conditions, and will often devolve from on type to another over time. Monitoring these progressions over time may provide valuable insight for characterizing these arrhythmias. In this thesis we presented data driven approaches for analyzing VAs in order to capture the dynamic signal characteristics to classify them and monitor their time progressions. A strong motivating factor for this work was to track the progression of VAs over time for the purpose of providing useful feedback in real emergency scenarios. By monitoring the progression of VAs over time, feedback may be given to EMS personnel when a change in the patient's VA has occurred, assisting in the selection and adaptation of appropriate treatment options.

The first objective was to extract non-instantaneous signal features to quantify the dynamic behaviour of the VAs over time and use them to assist in both long-term (in-hospital) and shortterm (out-of-hospital) classification of these VAs. The second objective was to use instantaneous TF features extracted from the HS to: classify VAs over the long-term, and provide near real-time feedback regarding the trends occurring in VAs over time. The results of these objectives will be 
explained below.

\subsection{Summary of Results}

Addressing the first objective of this thesis, dynamic features were extracted from the IMFs decomposed by EMD from the 60-s VA segments. The database consisted of 22 VT, 17 OVF, and 22 DVF segments which were verified by our collaborators at Toronto General Hospital. The features extracted were called ERV and ERS, and they were related to the underlying temporal complexity of the VA signals. These features were used for both long-term analysis designed to help classify the VAs for 'in-hospital' settings, and short-term analysis to assist medical personnel in monitoring these VAs 'out-of-hospital'. The IMF-energy based features were used to train an LDA-based classifier. A two-stage binary classification scheme was used, where initially VT was classified from VF, and subsequently the VF segments were sub-classified into OVF and DVF. In the initial stage, the classification accuracy achieved was $\mathbf{8 8 . 5 \%}$, and in the second stage the classification accuracy obtained was $\mathbf{8 0 \%}$. Since this approach was data driven, it is adaptable to the analysis of any other database of VAs, with the additional benefit of being highly computationally efficient.

For the short-term analysis, the same features were extracted over windowed 4-s segments of the total 60-s VA segments, and a cumulative averaging strategy was used to compare the progression of these features from one window to another. This method was tested on 20-s, 40-s, and 60-s

duration trials, and the proposed features performed better than comparative features for classifying OVF and DVF, a challenging task. This proposed approach has the potential to provide near real-time feedback to EMS personnel on the classification of VAs, and assist in the selection of the appropriate treatment option in emergency settings.

For the second objective of this thesis, instantaneous TF features were derived from the HS of the same 60-s VA segments as previously used. The instantaneous features extracted were: IF, $I B^{2}$ and $I E_{12}$. These features had the ability to track instantaneous changes occurring in the spectral and energy content of VAs over time, and were used for both long-term and short-term analysis. 
For long-term analysis, the instantaneous features were extracted and used to train an LDA-based classifier. The results for the VT vs. VF classification was $\mathbf{9 6 . 7 \%}$, and the results of the OVF vs. DVF classification was $\mathbf{8 1 \%}$. This approach improved upon the accuracy for VT/VF classification of the previous approach, namely due to the inclusion of frequency-based features in the analysis. Compared previous works that used TF analysis to study VAs, this approach was much less computationally expensive, and simpler to implement. Also, the HS has a number of advantages over other TFDs, including higher TF resolution, no cross-term interferences in the TF plane, and a more significant physiological meaning for its extracted instantaneous features like IF.

For the short-term analysis, EMD was performed on windowed segments of the total VA segments, starting with 4-s and expanding by a sliding 100-sample window. The HS was reconstructed for every segment, and the 3 instantaneous features were extracted to score each segment as VT, OVF or DVF. These scores were cumulatively averaged in order to capture the relative changes between windows over time. The instantaneous features benefited this approach by quantifying the changes happening locally within each window. This method was able to capture the trends visible in the total VA segments. The VA trend result presented showed a VA segment transitioning from PVT into OVF and finally DVF. This scenario could represent a patient whose condition was worsening over time, to the point where a defibrillatory shock may be necessary. If the EMS could see this progression happening, it would inform them that the VA is devolving and that a change in therapy may be required. Overall, this VA trend analysis has the powerful capability of providing near real-time feedback to EMS not only on the current state of a patient's VA, but the trend in which it is tending towards.

The final result of this thesis was the combination of the non-instantaneous and instantaneous features extracted previously for long-term analysis to further improve the maximum classification accuracy. Using a combination of features to train the LDA-based classifiers, the VT vs. VF classification accuracy achieved was $\mathbf{9 6 . 7 \%}$, and the OVF vs. DVF classification accuracy was 87\%. This result was an improvement on the results of using non-instantaneous and instantaneous 
features separately, and it surpassed the results of previous comparable works. This approach could be used by doctors and clinicians 'in-hospital' to analyze and diagnose patients and plan appropriate treatment options.

\subsection{Potential Applications}

Below is a short list of some potential applications for the methods presented in this thesis:

1. An adaptive, data driven pattern classifier for discriminating VAs 'in-hospital' for offline or retrospective analysis and 'out-of-hospital' scenarios.

2. A trend analysis algorithm that provides near real-time feedback on the progression of VAs over time to assist EMS in selecting and modifying treatment options in 'out-of-hospital' settings and for implantable devices such as ICDs.

\subsection{Future Works}

The goal of the methods proposed in this thesis was to assist medical personnel in diagnosing VAs so that appropriate therapy options can be chosen in both 'in-hospital' and 'out-of-hospital' settings. Although the results of these approaches were successful, there is always room for improvement. Additional EMD-based features that are computationally efficient can be explored to further improve the classification success. Other instantaneous TF features could also be investigated to gain further insight into quantifying the dynamic characteristics of VAs. The short-term analysis approaches shown may be programmed into ICDs to assist in improving the selection of therapies of patients while in-vivo. These algorithms could potentially be implemented into devices [65], which could be used by EMS in the field to help improve their VA diagnosis and optimize their selection and adaptation of treatment options. 


\section{Appendix A}

\section{HHT of Synthetic Signals}

The HHT of various synthetic signals is shown below for comparison to the VA ECG signals presented in this thesis. The HHT for sine wave, up-chirp, down-chirp, non-crossing chirp, and double-crossing chirp signals is shown below in Figure A.1. 


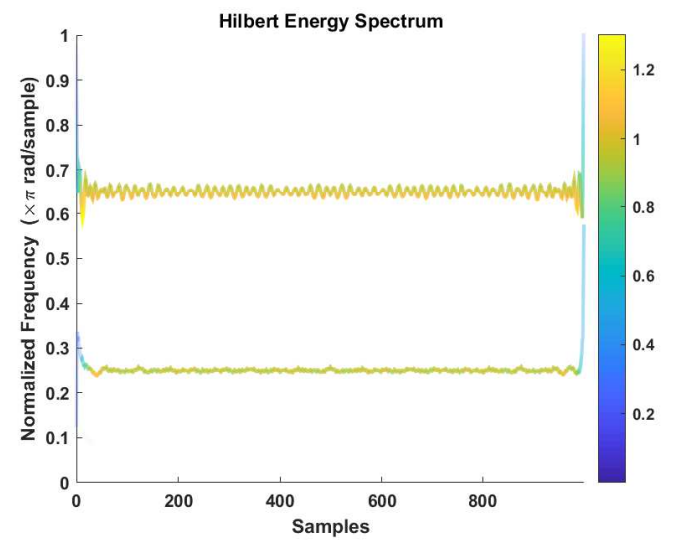

(a) Closely spaced sine wave signal.

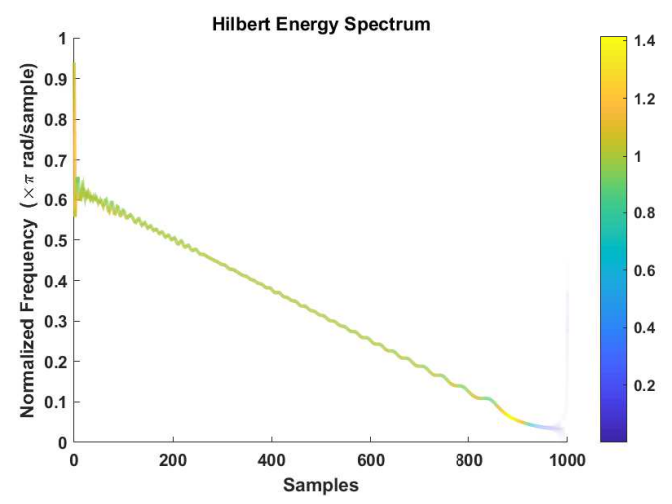

(c) Down-chirp signal.

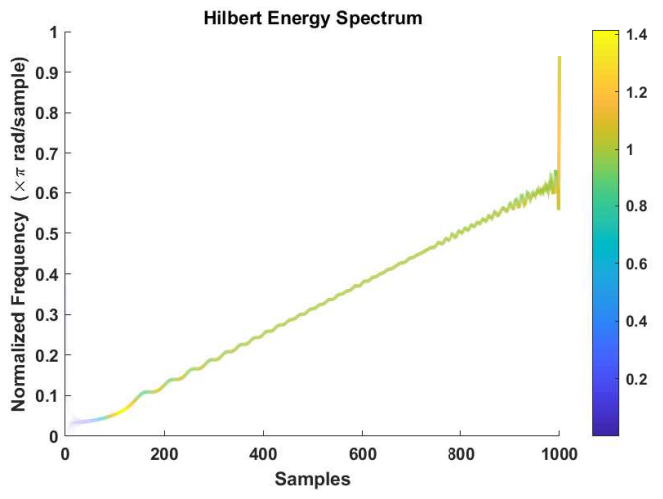

(b) Up-chirp signal.

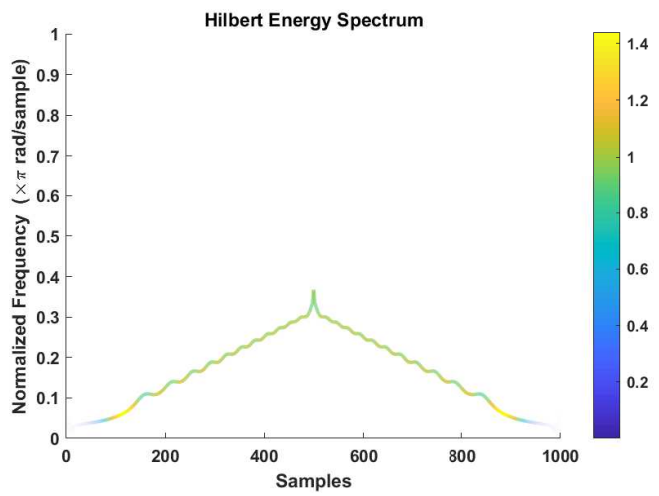

(d) Non-crossing chirp signal.

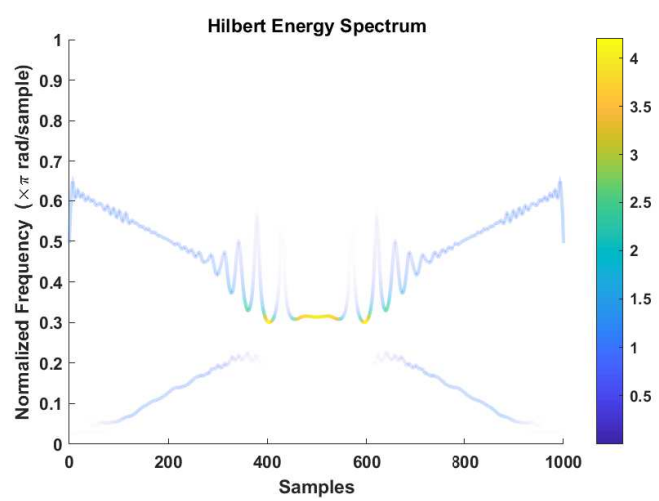

(e) Double-crossing chirp signal.

Figure A.1: The HS plots for various synthetic signals. 


\section{References}

[1] Heikki V Huikuri, Agustin Castellanos, and Robert J Myerburg. Sudden death due to cardiac arrhythmias. New England Journal of Medicine, 345(20):1473-1482, 2001.

[2] John E Hall. Guyton and Hall textbook of medical physiology. Saunders Elsevier, Philadelphia, PA, 2011.

[3] José Jalife. Ventricular fibrillation: mechanisms of initiation and maintenance. Annual review of physiology, 62(1):25-50, 2000.

[4] Abhijit Patwardhan, Sachin Moghe, Ke Wang, and Fabio Leonelli. Frequency modulation within electrocardiograms during ventricular fibrillation. American Journal of PhysiologyHeart and Circulatory Physiology, 279(2):H825-H835, 2000.

[5] Kang-An Cheng, Derek J Dosdall, Li Li, Jack M Rogers, Raymond E Ideker, and Jian Huang. Evolution of activation patterns during long-duration ventricular fibrillation in pigs. American Journal of Physiology-Heart and Circulatory Physiology, 302(4):H992-H1002, 2011.

[6] Kirsten HWJ Ten Tusscher, Rok Hren, and Alexander V Panfilov. Organization of ventricular fibrillation in the human heart. Circulation research, 100(12):e87-e101, 2007.

[7] Stephane Masse, Eugene Downar, Vijay Chauhan, Elias Sevaptsidis, and Kumaraswamy Nanthakumar. Ventricular fibrillation in myopathic human hearts: mechanistic insights from in vivo global endocardial and epicardial mapping. American Journal of Physiology-Heart and Circulatory Physiology, 292(6):H2589-H2597, 2007. 
[8] Krishnanand Balasundaram, Stéphane Masse, Krishnakumar Nair, and Karthikeyan Umapathy. A classification scheme for ventricular arrhythmias using wavelets analysis. Medical \& biological engineering \& computing, 51(1-2):153-164, 2013.

[9] Faramarz H Samie and Jose Jalife. Mechanisms underlying ventricular tachycardia and its transition to ventricular fibrillation in the structurally normal heart. Cardiovascular research, 50(2):242-250, 2001.

[10] Anton Amann, Robert Tratnig, and Karl Unterkofler. Reliability of old and new ventricular fibrillation detection algorithms for automated external defibrillators. Biomedical engineering online, 4(1):60, 2005.

[11] Hans-Ulrich Strohmenger, Karl H Lindner, and Charles G Brown. Analysis of the ventricular fibrillation ecg signal amplitude and frequency parameters as predictors of countershock success in humans. Chest, 111(3):584-589, 1997.

[12] Leon Cohen. Time-frequency distributions-a review. Proceedings of the IEEE, 77(7):941981, 1989.

[13] RH Clayton, RWF Campbell, and A Murray. Time-frequency analysis of human polymorphic ventricular tachycardia. In Computers in Cardiology 1997, pages 97-100. IEEE, 1997.

[14] Muhammad Abdullah Arafat, Jubair Sieed, and Md Kamrul Hasan. Detection of ventricular fibrillation using empirical mode decomposition and bayes decision theory. Computers in Biology and Medicine, 39(11):1051-1057, 2009.

[15] Baodan Bai and Yuanyuan Wang. Ventricular fibrillation detection based on empirical mode decomposition. In Bioinformatics and Biomedical Engineering,(iCBBE) 2011 5th International Conference on, pages 1-4. IEEE, 2011.

[16] Yung-Hung Wang, Chien-Hung Yeh, Hsu-Wen Vincent Young, Kun Hu, and Men-Tzung Lo. On the computational complexity of the empirical mode decomposition algorithm. Physica A: Statistical Mechanics and its Applications, 400:159-167, 2014. 
[17] Z Huang, Y Chen, and M Pan. Time-frequency characterization of atrial fibrillation from surface ecg based on hilbert-huang transform. Journal of medical engineering \& technology, 31(5):381-389, 2007.

[18] Sachin Anil Moghe. Spatio-temporal variation in activation intervals during ventricular fibrillation. University of Kentucky Doctoral Dissertations, 2002.

[19] Karthikeyan Umapathy, Stephane Massé, Elias Sevaptsidis, John Asta, Sidhar Krishnan, and Kumaraswamy Nanthakumar. Spatiotemporal frequency analysis of ventricular fibrillation in explanted human hearts. IEEE Transactions on Biomedical Engineering, 56(2):328-335, 2009.

[20] Matthew Hotradat, Krishnanand Balasundaram, Stephane Masse, Krishnakumar Nair, Kumaraswamy Nanthakumar, and Karthikeyan Umapathy. Instantaneous time-frequency features in characterizing ventricular arrhythmias using empirical mode decomposition. In 2018 52nd Asilomar Conference on Signals, Systems and Computers. IEEE, 2018 (In Press).

[21] Roy M John, Usha B Tedrow, Bruce A Koplan, Christine M Albert, Laurence M Epstein, Michael O Sweeney, Amy Leigh Miller, Gregory F Michaud, and William G Stevenson. Ventricular arrhythmias and sudden cardiac death. The Lancet, 380(9852):1520-1529, 2012.

[22] Karthikeyan Umapathy, Sridhar Krishnan, Vijay Parsa, and Donald G Jamieson. Discrimination of pathological voices using a time-frequency approach. IEEE Transactions on Biomedical Engineering, 52(3):421-430, 2005.

[23] Stéphane Mallat. A wavelet tour of signal processing: the sparse way. Academic press, 3rd edition, 2009.

[24] RH Clayton and A Murray. Comparison of techniques for time-frequency analysis of the ecg during human ventricular fibrillation. IEE Proceedings-Science, Measurement and Technology, 145(6):301-306, 1998. 
[25] Fredrick G Evans, Jack M Rogers, William M Smith, and Raymond E Ideker. Automatic detection of conduction block based on time-frequency analysis of unipolar electrograms. IEEE transactions on biomedical engineering, 46(9):1090-1097, 1999.

[26] Bum-Rak Choi, Wonchul Nho, Tong Liu, and Guy Salama. Life span of ventricular fibrillation frequencies. Circulation Research, 91(4):339-345, 2002.

[27] L Angrisani, P Daponte, M D'apuzzo, and A Testa. A measurement method based on the wavelet transform for power quality analysis. IEEE Transactions on Power Delivery, 13(4):990-998, 1998.

[28] JS Karlsson, Björn Gerdle, and M Akay. Analyzing surface myoelectric signals recorded during isokinetic contractions. IEEE Engineering in Medicine and Biology Magazine, 20(6):97$105,2001$.

[29] Paul S Addison. Wavelet transforms and the ecg: a review. Physiological measurement, 26(5):R155, 2005.

[30] M. Rasooli et al. Analysis of electrocardiogram pre-shock waveforms during ventricular fibrillation. Biomed Signal Process Control, 21:26-33, 2015.

[31] Hiroshi Endoh, Seiji Hida, Satomi Oohashi, Yusuke Hayashi, Hidenori Kinoshita, and Tadayuki Honda. Prompt prediction of successful defibrillation from 1-s ventricular fibrillation waveform in patients with out-of-hospital sudden cardiac arrest. Journal of anesthesia, 25(1):34-41, 2011.

[32] U Rajendra Acharya, Hamido Fujita, Oh Shu Lih, Yuki Hagiwara, Jen Hong Tan, and Muhammad Adam. Automated detection of arrhythmias using different intervals of tachycardia ecg segments with convolutional neural network. Information sciences, 405:81-90, 2017.

[33] Ali Bahrami Rad, Trygve Eftestøl, Kjersti Engan, Unai Irusta, Jan Terje Kvaløy, Jo KramerJohansen, Lars Wik, and Aggelos K Katsaggelos. Ecg-based classification of resuscitation 
cardiac rhythms for retrospective data analysis. IEEE Transactions on Biomedical Engineering, 64(10):2411-2418, 2017.

[34] Leon Cohen. Time-frequency analysis, volume 778. Prentice hall, 1995.

[35] HH Huang, HL Chan, PL Lin, CP Wu, and CH Huang. Time-frequency spectral analysis of heart rate variability during induction of general anaesthesia. British journal of anaesthesia, 79(6):754-758, 1997.

[36] François Auger, Patrick Flandrin, Paulo Gonçalvès, and Olivier Lemoine. Time-frequency toolbox. CNRS France-Rice University, 46, 1996.

[37] MJ Ebden, L Tarassenko, SJ Payne, A Darowski, and JD Price. Time-frequency analysis of the ecg in the diagnosis of vasovagal syndrome in older people. In Engineering in Medicine and Biology Society, 2004. IEMBS'04. 26th Annual International Conference of the IEEE, volume 1, pages 290-293. IEEE, 2004.

[38] John OToole, Mostefa Mesbah, and Boualem Boashash. A discrete time and frequency wigner-ville distribution: properties and implementation. In Proc. Int. Conf. on Digital Signal Processing and Comm. Systems, volume 400, pages 19-21, 2005.

[39] Mokhtar Mohammadi, Ali Akbar Pouyan, Nabeel Ali Khan, and Vahid Abolghasemi. An improved design of adaptive directional time-frequency distributions based on the radon transform. Signal Processing, 150:85-89, 2018.

[40] Norden E Huang, Zheng Shen, Steven R Long, Manli C Wu, Hsing H Shih, Quanan Zheng, Nai-Chyuan Yen, Chi Chao Tung, and Henry H Liu. The empirical mode decomposition and the hilbert spectrum for nonlinear and non-stationary time series analysis. In Proceedings of the Royal Society of London A: mathematical, physical and engineering sciences, volume 454, pages 903-995. The Royal Society, 1998.

[41] M. Kaleem, B. Ghoraani, A. Guergachi, and S. Krishnan. Pathological speech signal analysis 
and classification using empirical mode decomposition. Medical \& biological engineering \& computing, 51:811-821, 2013.

[42] Zhaohua Wu and Norden E Huang. Ensemble empirical mode decomposition: a noiseassisted data analysis method. Advances in adaptive data analysis, 1(01):1-41, 2009.

[43] U Rajendra Acharya, S Vinitha Sree, G Swapna, Roshan Joy Martis, and Jasjit S Suri. Automated eeg analysis of epilepsy: a review. Knowledge-Based Systems, 45:147-165, 2013.

[44] Maria E Torres, Marcelo A Colominas, Gastón Schlotthauer, and Patrick Flandrin. A complete ensemble empirical mode decomposition with adaptive noise. In Acoustics, speech and signal processing (ICASSP), 2011 IEEE international conference on, pages 4144-4147. IEEE, 2011.

[45] Jia-Rong Yeh, Jiann-Shing Shieh, and Norden E Huang. Complementary ensemble empirical mode decomposition: A novel noise enhanced data analysis method. Advances in adaptive data analysis, 2(02):135-156, 2010.

[46] Konstantin Dragomiretskiy and Dominique Zosso. Variational mode decomposition. IEEE transactions on signal processing, 62(3):531-544, 2014.

[47] RK Tripathy, LN Sharma, and Samarendra Dandapat. Detection of shockable ventricular arrhythmia using variational mode decomposition. Journal of medical systems, 40(4):79, 2016.

[48] Norden E Huang, Xianyao Chen, Men-tzung Lo, and Zhaohua Wu. On hilbert spectral representation: a true time-frequency representation for nonlinear and nonstationary data. Advances in adaptive data analysis, 3(01n02):63-93, 2011.

[49] Zuocai Wang. Hilbert transform applications in signal analysis and non-parametric identification of linear and nonlinear systems. 2011.

[50] François Auger, Patrick Flandrin, Paulo Gonçalvès, and Olivier Lemoine. Time-frequency toolbox. CNRS France-Rice University, 46, 1996. 
[51] Patrick J Loughlin and Keith L Davidson. Modified cohen-lee time-frequency distributions and instantaneous bandwidth of multicomponent signals. IEEE Transactions on Signal Processing, 49(6):1153-1165, 2001.

[52] Adriano O Andrade, Peter Kyberd, and Slawomir J Nasuto. The application of the hilbert spectrum to the analysis of electromyographic signals. Information Sciences, 178(9):21762193, 2008.

[53] Mark A Poletti. The development of instantaneous bandwidth via local signal expansion. Signal processing, 31(3):273-281, 1993.

[54] MB Malarvili, I Kamarulafizam, S Hussain, and D Helmi. Heart sound segmentation algorithm based on instantaneous energy of electrocardiogram. In Computers in Cardiology, 2003, pages 327-330. IEEE, 2003.

[55] Yi Li, Fan Yingle, Li Gu, and Tong Qinye. Sleep stage classification based on eeg hilberthuang transform. In Industrial Electronics and Applications, 2009. ICIEA 2009. 4th IEEE Conference on, pages 3676-3681. IEEE, 2009.

[56] Richard O Duda, Peter E Hart, David G Stork, et al. Pattern classification. 2nd. Edition. New York, 55, 2001.

[57] Tom Fawcett. An introduction to roc analysis. Pattern recognition letters, 27(8):861-874, 2006.

[58] Ary L Goldberger, Luis AN Amaral, Leon Glass, Jeffrey M Hausdorff, Plamen Ch Ivanov, Roger G Mark, Joseph E Mietus, George B Moody, Chung-Kang Peng, and H Eugene Stanley. Physiobank, physiotoolkit, and physionet: components of a new research resource for complex physiologic signals. Circulation, 101(23):e215-e220, 2000.

[59] Amir Eftekhar, Christofer Toumazou, and Emmanuel M Drakakis. Empirical mode decomposition: Real-time implementation and applications. Journal of Signal Processing Systems, 73(1):43-58, 2013. 
[60] Gang Wang, Xian-Yao Chen, Fang-Li Qiao, Zhaohua Wu, and Norden E Huang. On intrinsic mode function. Advances in Adaptive Data Analysis, 2(03):277-293, 2010.

[61] RT Rato, Manuel Duarte Ortigueira, and AG Batista. On the hht, its problems, and some solutions. Mechanical Systems and Signal Processing, 22(6):1374-1394, 2008.

[62] Marzieh Rasooli. Blind source separation in the analysis of electrocardiogram pre-shock waveforms during ventricular fibrillation. Theses and Dissertations, Ryerson University, 2013.

[63] KP Ajitha Gladis and K Ramar. A novel method for content based image retrieval using the approximation of statistical features, morphological features and bpn network. In Conference on Computational Intelligence and Multimedia Applications, 2007. International Conference on, volume 3, pages 179-184. IEEE, 2007.

[64] Shu-Sen Chang, David Gunnell, Jonathan AC Sterne, Tsung-Hsueh Lu, and Andrew TA Cheng. Was the economic crisis 1997-1998 responsible for rising suicide rates in east/southeast asia? a time-trend analysis for japan, hong kong, south korea, taiwan, singapore and thailand. Social science \& medicine, 68(7):1322-1331, 2009.

[65] Ying-Yi Hong and Yu-Qing Bao. Fpga implementation for real-time empirical mode decomposition. IEEE Transactions on Instrumentation and Measurement, 61(12):3175-3184, 2012. 


\section{Glossary}

CEEMDAN - Complete Ensemble Empirical Mode Decomposition with Adaptive Noise CPR - Cardiopulmonary Resuscitation

ECG - Electrocardiogram

EEMD - Ensemble Empirical Mode Decomposition

EMD - Empirical Mode Decomposition

EMS - Emergency Medical Staff

ERS - Energy Ratio Skew

ERV - Energy Ratio Variance

HHT - Hilbert-Huang Transform

HS - Hilbert Energy Spectrum

IB $^{2}$ - Squared Instantaneous Bandwidth

ICD - Implantable Cardioverter-Defibrillator

IE - Instantaneous Energy

IF - Instantaneous Mean Frequency

IMF - Intrinsic Mode Function

LDA - Fisher's Linear Discriminant Analysis

LOOCV - Leave-One-Out Cross-Validation

NSR - Normal Sinus Rhythm

PVT - Polymorphic Ventricular Tachycardia

ROC - Receiver Operating Characteristic

SCD - Sudden Cardiac Death

VA - Ventricular Arrhythmia

VF - Ventricular Fibrillation

VT - Ventricular Tachycardia 